\title{
Grainyhead-like 2 Sensitizes Cells to Natural Killer Cell Cytotoxicity and Promotes the Interferon Response
}

\author{
Ian Philip MacFawn \\ West Virginia University, ipm0002@mix.wvu.edu
}

Follow this and additional works at: https://researchrepository.wvu.edu/etd

Part of the Cancer Biology Commons

\section{Recommended Citation}

MacFawn, Ian Philip, "Grainyhead-like 2 Sensitizes Cells to Natural Killer Cell Cytotoxicity and Promotes the Interferon Response" (2020). Graduate Theses, Dissertations, and Problem Reports. 7778.

https://researchrepository.wvu.edu/etd/7778

This Dissertation is protected by copyright and/or related rights. It has been brought to you by the The Research Repository @ WVU with permission from the rights-holder(s). You are free to use this Dissertation in any way that is permitted by the copyright and related rights legislation that applies to your use. For other uses you must obtain permission from the rights-holder(s) directly, unless additional rights are indicated by a Creative Commons license in the record and/ or on the work itself. This Dissertation has been accepted for inclusion in WVU Graduate Theses, Dissertations, and Problem Reports collection by an authorized administrator of The Research Repository @ WVU.

For more information, please contact researchrepository@mail.wvu.edu. 
Graduate Theses, Dissertations, and Problem Reports

2020

Grainyhead-like 2 Sensitizes Cells to Natural Killer Cell

Cytotoxicity and Promotes the Interferon Response

Ian Philip MacFawn

Follow this and additional works at: https://researchrepository.wvu.edu/etd

Part of the Cancer Biology Commons 
Grainyhead-like 2 Sensitizes Cells to Natural Killer Cell Cytotoxicity and Promotes the Interferon Response

Ian Philip MacFawn

Dissertation submitted

to the School of Medicine

at West Virginia University

in partial fulfillment of the requirements for the degree of

Doctor of Philosophy in

Cancer Cell Biology

\author{
Steven Frisch, Ph.D., Mentor \\ Timothy Eubank, Ph.D., Chair \\ Lori Hazlehurst, Ph.D. \\ Aaron Robart, Ph.D. \\ Christopher Cifarelli, MD, Ph.D. \\ Michael Schaller, Ph.D.
}

Department of Biochemistry

Morgantown, West Virginia

2020

Keywords: GRHL2, Epithelial to mesenchymal transition, Natural Killer Cell, Interferon Copyright 2018 Ian Philip MacFawn 


\begin{abstract}
Grainyhead-like 2 Sensitizes Cells to Natural Killer Cell Cytotoxicity and Promotes the Interferon Response

Ian Philip MacFawn

Our research determined that the epithelial master transcription factor Grainyhead-like 2 (GRHL2) promotes sensitivity to Natural Killer (NK) cell-mediated killing, and modulates the interferon I (IFN-I) response of epithelial cells. Immune surveillance by NK cells constitutes a major selective pressure for circulating tumor cells. Epithelial (GRHL2-expressing) cells exhibited significantly higher rates of NK conjugation, a crucial step in direct cell-mediated cytotoxicity. Mechanistically, GRHL2 upregulates expression of intercellular adhesion molecule 1 (ICAM-1), a cell surface molecule critical for NK to target cell synaptogenesis. GRHL2 epigenetically regulates gene expression, and we found that GRHL2 mutant proteins unable to interact with the epigenetic modifiers p300 or KMT2C/D exhibited altered NK sensitivity, and ICAM-1 expression was lost. In summary, the epithelial state, enforced by GRHL2, kept these cells susceptible to NK-mediated cytolysis. Further, our data suggest GRHL2 critically promotes IFN I production in response to a double strand RNA mimetic. The results from these experiments identify novel roles for GRHL2 in maintaining the relationship between epithelial cells and the innate and adaptive arms of the immune system.
\end{abstract}


Table of Contents

1.

Chapter I: GRHL2 sensitizes cell to Natural Killer cell cytotoxicity through epigenetic mechanisms.

1. Chapter I: Introduction

16. Chapter I: Results

16. I.a. GRHL2 sensitizes cells to NK92 cytotoxicity

20.............b. The effect of Inhibitory/Activating NK Ligand Expression and Fadd knockdown on NK Killing

22................. The effect of epithelial versus mesenchymal ECM on NK92 killing.

24...............1.c. GRHL2 suppresses expression of the proteoglycan Serglycin

27. I.d. GRHL2 suppresses autophagy in HT1080 cells

29.............e GRHL2 promotes NK92/target cell conjugation through upregulation of ICAM-1

35.................1.f. GRHL2 regulates NK sensitivity through epigenetic interactions.

44............... EMT signature anti-correlates with Interferon signature in Lung Adenocarcinoma patient samples (TCGA).

46........1.h. GRHL2 protein expression correlates with time to relapse in Breast cancer

51 Chapter I: Conclusion

55 Chapter II. GRHL2 Promotes Interferon Signaling in Epithelial Cells.

55. Chapter II: Introduction

63. Chapter II: Results

63. ..II.a. GRHL2 regulates subset of interferon response genes (ISGs).

66. .II.b. GRHL2 is required for Type I and III IFN production in Mcf10aneoT cells.

72. II.c. GRHL2 sensitizes cells to poly(I:C) and is downregulated by IFN- $\gamma$. 
75.

..Chapter II: Conclusion

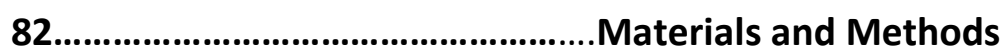

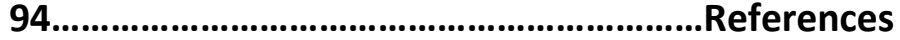




\section{Chapter I: GRHL2 sensitizes cell to Natural Killer cell cytotoxicity through epigenetic mechanisms.}

\section{Chapter I: Introduction}

Perturbation of genetic homeostasis in epithelial cells is a significant driver of tumorigenesis; over $90 \%$ of cancers are classified as "carcinoma", which arise from epithelial origin. Epithelial cells are one of many final products of a long and tightly regulated process of differentiation that begins in embryonic stem cells during early mammalian development. In order to limit the self-renewing, pluripotent characteristics of embryonic stem cells, master transcription factors must be turned on, or off, in a concerted manner in order to gradually narrow the potential lineage-specific gene expression programs of different subsets of the rapidly proliferating embryo. Thus, the "terminally" differentiated epithelial cell which comprises various tissue across the body is locked into its identity by the concerted influence of epithelial master transcription factors which promote the expression of epithelial genes (A. F. Chen et al., 2018; Soares \& Zhou, 2018), and antagonize the expression and activity of various stemness or mesenchymal master transcription factors. A great body of scientific literature has illustrated the oncogenic characteristics that arise from imbalance of epithelial-mesenchymal transcriptional control (Nieto, Huang, Jackson, \& Thiery, 2016; Ribatti, Tamma, \& Annese, 2020). These include resistance to chemotherapeutics, increased invasion and motility, anoikisresistance, phenotypic plasticity, aberrant immune response, metabolic reprogramming, and more. Hence, promoting the epithelial "default" state discourages some types of oncogenic progression and represents a lynchpin for understanding tumor biology (Frisch, 1997). 
Grainyhead-like 2 (GRHL2) is considered an epithelial master transcription factor. Acting as a pioneer transcription factor, GRHL2 localizes to genomic loci and binds closed chromatin regions, subsequently recruiting chromatin remodeling enzymes in order to alter histone modifications, thereby loosening repressive chromatin and providing topography permissive for transcription factor binding (Jacobs et al., 2018; Reese, Harrison, \& Alarid, 2019). Many canonical epithelial genes contain GRHL2 binding sites in their regulatory regions including CDH1, ELF3, OVOL2, RAB25, p63, CLDN4 (Frisch, Farris, \& Pifer, 2017) . Pertaining to epigenetic regulation, GRHL2 has, at least, a DNA-binding region, a region which inhibits the histone acetyltransferase p300 (AA's 425-437) and a direct interaction with the lysine methyltransferases KMT2C and KMT2D (AA's 32-33) (Diagram 1) (MacFawn et al., 2019; Pifer et al., 2016). GRHL2 plays an indispensable role in early development by overseeing the human embryonic stem cell (hESC) to epiblast-like cell transition. During this time, GRHL2 becomes active, assumes control of target enhancers, and potentiates epithelial gene expression as the hESC transitions away from pluripotency towards a more differentiated "epiblast-like cell": an early cell state that gives rise to subsequent epithelial cell lineages (A. F. Chen et al., 2018).

The gene was originally described in Drosophila melanogaster: descriptively termed "Grainyhead" following observations of a granular, sclerotic head phenotype in Grainyhead mutant flies. These flies also exhibited altered epidermal barrier formation and wound response, as well as an inability to properly control developmental genes normally manifested through promoter binding of ultrabithorax $(u b x)$, engrailed, dopa carboxylase $(D d c)$ and the $D$. melanogaster E-cadherin homologue shotgun (Dynlacht, Attardi, Admon, Freeman, \& Tjian, 1989). D. melanogaster Grainyhead protein expression is temporally- and spatially-restricted to 
ectoderm lineages during development, with detectable expression in epidermis, central nervous system (CNS), and gut (Bray, Burke, Brown, \& Hirsh, 1989). Subsequent identification of the mammalian homologues revealed a family of "Grainyhead-like" genes: GRHL1, GRHL2 and GRHL3. These three genes code for structurally similar proteins with a conserved Nterminal transactivation domain, a p53-like DNA-binding domain, and a C-terminal dimerization domain, however the functional roles of these proteins are diverse and mostly non-redundant (Frisch et al., 2017).

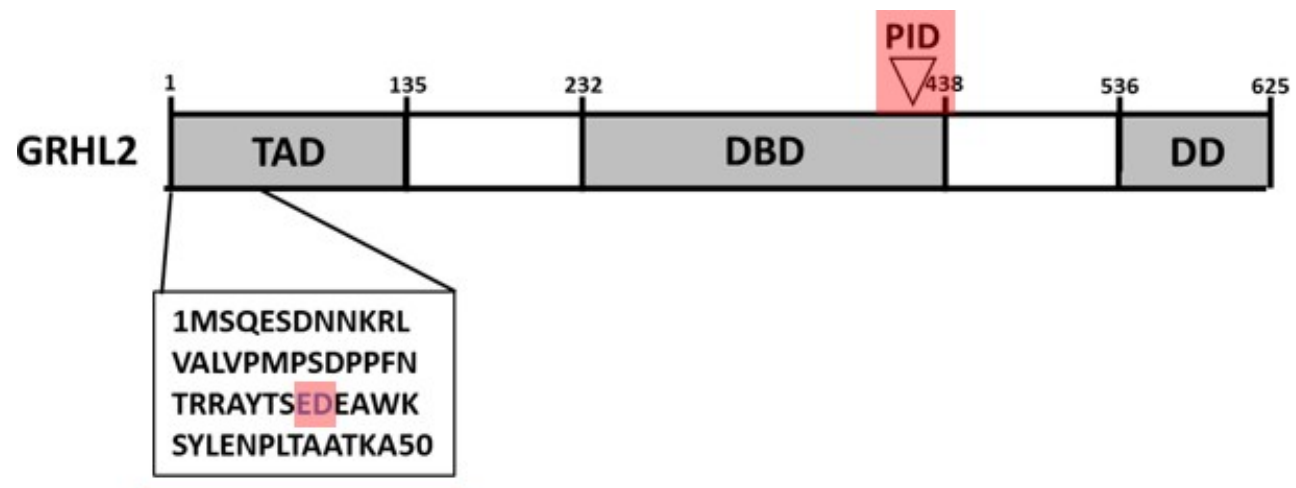

\section{KMT interaction}

Diagram 1. GRHL2 Protein Domain Map:

TAD: Transactivation domain

DBD: DNA-binding domain

DD: Dimerization domain

PID: p300 inhibiting region (Pifer et al., 2016)

$E D \rightarrow A A K M T 2 C / K M T 2 D$ interaction region (MacFawn et al., 2019)

Owing to its prolific and widespread support of epithelial gene expression, downregulation or knockout of GRHL2 by various endogenous or ectopic methods is consequential for a wide variety of cellular characteristics and functions. In mice, GRHL2-/mice 
exhibit insufficient neural tube closure, epidermal defects and epithelial migration problems causing embryonic lethality at day E11.5 (Boglev et al., 2011; Ray \& Niswander, 2016). GRHL2 also regulates epithelial cells of the lungs, with expression in luminal and basal p63 ${ }^{+} / \mathrm{KRTS}^{+}$ epithelium, while also forming a positive feedback loop with Nkx2-1 in alveolar type II cells, disruption of which leads to flattening and loss of cuboidal cell shape (Varma et al., 2012). GRHL2 was subsequently shown to orchestrate epithelial morphogenesis, polarity, and differentiation of basal stem cells in models of airway mucociliary epithelium (Gao, Bali, Randell, \& Hogan, 2015). Kidney development and tubulogenesis are also impacted by loss of GRHL2, causing reduced collecting duct luminal expansion, reduced epithelial barrier integrity and rescue of tubulogenesis, respectively (Aue et al., 2015; Pifer et al., 2016).

In addition to its integral role in specific tissue and organ development, GRHL2 modulation has implications in cancer. The specific roles of Grainyhead-like proteins in cancer have been reviewed extensively (Frisch et al., 2017; He et al., 2020; Kotarba, TarachaWisniewska, \& Wilanowski, 2020; Reese et al., 2019). Low GRHL2 expression, according to IHC, in a subset of breast cancer cells correlates with worse prognosis and lymph node metastasis, however, GRHL2 can also increase proliferation in breast cancer cell lines (Werner et al., 2013). Further, it was found that GRHL2 expression is absent in metastatic, "basal B" type breast cancer cell lines MDA-MB-231 and BT-549 cells, while in other breast cancer cell lines GRHL2 was found to positively regulate erbb3 expression: a family member of erbb2 linked with poor outcomes in breast cancer (Werner et al., 2013). In colorectal cancer, GRHL2 knockdown increased metastasis in mouse models, via induction of EMT, but high expression was also positively associated with clinical stage and tumor size (Quan et al., 2015; Z. Yang, Wu, Chen, 
Min, \& Quan, 2019). In NSCLC, while high expression of GRHL2 correlates with worse prognosis, in vitro investigation found that while GRHL2 promotes proliferation and colony formation, it also inhibits metastatic attributes (Pan et al., 2017). GRHL2's role in suppressing oncogenic EMT would point to a widespread function as a tumor suppressor, however, analysis of patient data accumulated by TCGA and other repositories, as well as in vitro and in vivo experiments exploring GRHL2's effect on tumor progression reveals a highly context-dependent role for GRHL2.

Tumor recurrence and metastasis remain the prevalent causes of death for cancer patients. Multiple mechanisms can lead to selection of resistant cells, including tumor immuneediting, persistence of drug-resistant subclones/cancer stem cells, phenotypic heterogeneity, and EMT (Dudas, Ladanyi, Ingruber, Steinbichler, \& Riechelmann, 2020; F. Li, Tiede, Massagué, \& Kang, 2007; Smith \& Bhowmick, 2016). These can contribute to the selective process of tumor evolution, which winnows the composition of a tumor and can promote recurrence and metastatic spread to secondary sites in the body. Lately, much research emphasis has been directed towards understanding mechanisms that allow carcinoma cells to evade tumor immune surveillance and/or promote immunological tolerance. Incisive studies conducted by the Weinberg lab explored the spatial and cellular composition of the tumor immune microenvironment in a mouse adenocarcinoma MMTV-PyMT model (Dongre et al., 2017). Tumors from this model were isolated; their cells characterized and sorted based upon epithelial or mesenchymal features. Compared with epithelial (E-cadherin/EpCAM ${ }^{\text {hi }}$ ) cells, orthotopically re-introduction of mesenchymal cells (Vimentin/EpCAM ${ }^{\text {lo }}$ ) produced tumors containing more immunosuppressive $\mathrm{CD} 25^{+} \mathrm{FOXP} 3^{+}$Tregs and $\mathrm{M} 2$ polarized macrophages, 
particularly in instances where the EMT TF Snail expression was high. The tumors arising from re-implanted epithelial cells contained more $\mathrm{CD}^{+} \mathrm{T}$ cells associated with effector function (GrzB, perf, IFN- $\gamma$ ) compared with the mesenchymal tumors which had numerically reduced $\mathrm{CD}^{+} \mathrm{T}$ cells that also exhibited higher PD-1. Interestingly, spatial analysis of co-injected epithelial and mesenchymal cells showed that the two cell types segregated in the resulting tumor, and that Arginase ${ }^{+} \mathrm{M} 2$ macrophages preferentially associated with mesenchymal regions, while $\mathrm{CD} 8^{+} \mathrm{T}$ cells were found in proximity to epithelial regions (Dongre et al., 2017). These observations support the idea that tumor cells undergoing EMT may be more refractory to anti-tumor immunity, and may also create a sheltering immunosuppressive environment.

Because of its ability to reinforce the epithelial state, thereby discouraging EMT, GRHL2's ability to act in the capacity of a tumor suppressor has been previously investigated. GRHL2 antagonizes TGF- $\beta$-mediated signaling, reducing mammosphere formation and invasive capacity, while increasing BMP2 in HMLE cells. Further, Zeb1 has well-documented oncogenic effects in breast cancer and promotes plasticity and metastasis in pancreatic cancer; GRHL2 has been shown to repress Zeb1 in vitro (Cieply et al., 2012; Krebs et al., 2017; H.-T. Wu et al., 2020). GRHL2 is also able to inhibit the metabolic switch towards oxidative phosphorylation often exhibited by cancer cells. Related to this, GRHL2 expression, in contrast to cells that have undergone EMT, suppresses the metabolic enzyme GLUD1, thereby maintaining intracellular ROS levels, helping cells to maintain sensitivity to anoikis (Farris et al., 2016).

Regarding epigenetic regulation, GRHL2 inhibits the histone acetyltransferase (HAT) activity of p300, contextually, at mesenchymal gene regulatory regions. Re-expression of GRHL2 in a mesenchymal subpopulation (MSP) (CD44hi/CD24lo) of HMLE cells causes repression of 
various matrix metalloproteinase (MMPs), likely attributable to reduced p300-deposited H3K27ac at promoters, as experimentally observed with MMP1, MMP2 and MMP14 (Pifer et al., 2016). The inhibition of p300 HAT activity is notable; while this capability is also shared by adenoviral E1A protein, the vast majority of transcription factors (>400) associating with p300 are thought to mainly use it as a coactivator, rather than inhibiting it. GRHL2 joins E1A-like inhibitor of differentiation (EID) proteins as unique inhibitors of p300 function (Pifer et al., 2016). Expanding the mechanism of GRHL2's transcriptional control, preliminary yeast two hybrid interaction data produced previously in our lab, and experiments conducted by other researchers, suggest that the two paralogous lysine methyltransferases $2 \mathrm{C}$ and D (KMT2C, KMT2D), also known as MLL3 and MLL2, could interact with GRHL2. KMT2C and KMT2D are members of the MLR subgroup of the KMT2 protein family. They are high molecular weight proteins (4915aa, 5537aa) and contain a catalytic SET domain for lysine methylation, their namesake, among other domains. KMT2C and KMT2D join a conglomeration of epigenetic regulatory proteins as part of the COMPASS complex (Complex of Proteins Associated with Set1) (Fagan \& Dingwall, 2019). SET domain mutation is frequently observed in KMT2A and KMT2C, suggesting that stability of their lysine methyltransferase activity is consequential in tumor development (Weirich, Kudithipudi, Kycia, \& Jeltsch, 2015). Indeed, KMT2C and KMT2D are two of the most commonly occurring mutated genes across all cancer types; to illustrate, KMT2C is found to be mutated in $8 \%-11 \%$ of breast cancer patients (Ellis et al., 2012; Fagan \& Dingwall, 2019; Wang et al., 2011), with a reduction in mRNA levels in 43\% of patients (Wang et al., 2011). In estrogen receptor positive (ER+) breast cancer KMT2C is specifically implicated in supporting the ER-collaborating pioneer transcription factor FOXA1. FOXA1 utilizes KMT2C's 
H3K4me1 (monomethylation) function to modify enhancer regions of target genes, permitting transcription. In this study, rapid immunoprecipitation mass spectroscopy of endogenous proteins (RIME) also revealed GRHL2 as a top predicted associator with KMT2C (Jozwik, Chernukhin, Serandour, Nagarajan, \& Carroll, 2016). A direct interaction between KMT2C/D and GRHL2 was tested and well supported by data produced previously in our lab. Understanding the interplay between GRHL2 and these epigenetic interactors is important in understanding how it influences epithelial identity, as well as repressing or reversing EMT. By reinforcing epithelial gene expression programs, GRHL2 preserves particular functions and responses across multiple cellular systems with demonstrable in vitro tumor suppressive effects.

The process of EMT is thought to be intertwined with the metastatic potential of many cancer types. Many barriers exist to reduce the likelihood of a cell's breaking away from its primary tissue site which may lead to metastasis, however, induction of various degrees of mesenchymal transition can equip a cell with tools to overcome these barriers (Pastushenko \& Blanpain, 2019). EMT can cause cells to become more motile and invasive, dissolve surrounding matrix and resist anoikis (Pearson, 2019) (Cieply et al., 2012). Entering the circulatory system constitutes another major selective pressure on newly departed circulating tumor cells (CTCs). The number of circulating tumor cells shed from a primary site vastly exceeds the number of metastatic lesions, underscoring the tremendous negative selective pressures levied on disseminating tumor cells (Massagué \& Obenauf, 2016).

Circulating immune cells, particularly Natural Killer (NK) cells, surveille cells and can effectively destroy most virally infected or transformed cells (Lopez-Soto, Gonzalez, Smyth, \& Galluzzi, 2017). NK cells share a common precursor with, and are the most populous members 
of, the Innate Lymphoid Cells (ILCS). This clade is defined by a lack of lineage markers (lin ${ }^{-}$) and absence of antigen receptor expression; nonetheless, NK cells develop cytotoxic effector function while the other phylogenetic branches of this group: ILC1, ILC2 and ILC3, do not, and are specialized cytokine producing cells (Artis \& Spits, 2015). No exclusively definitive marker of NK cell identity has been discovered; they share an amalgamation of markers overlapping with other immune cell subsets of the innate and adaptive arms. Nonetheless, they can be divided into two general populations: a CD16+ CD56 dim cytotoxic subset, and a CD16- CD56 bright cytokine secreting subset (Poli et al., 2009). These two subsets represent the prevalent functions of NK cells: to rapidly engage and kill target cells (Granzyme B, Perforin.; FasLigand, Trail; Antibody Dependent Cell-Mediated Cytotoxicity), and to secrete various cytokines including IFN- - , GM-CSF, TNF and other growth factors. NK cells are uniquely tailored to surveille cells that are under the insidious influence of viral infection or malignant transformation (Mancini \& Vidal, 2020; M. G. Morvan \& L. L. Lanier, 2016). To this end they express a variety of activating and inhibitory receptors; the prevailing net signal will dictate the NK cell's response upon recognition of a possible target cell. In contrast to CD8+ T cell dependence upon target cell MHC Class I expression for their cell-mediated cytotoxicity, NK recognition of an MHC Class I molecule through Killer immunoglobulin receptor (KIR) or CD94NKG2C receptors will inhibit killing; they are not antigen-restricted (Mancini \& Vidal, 2020). This "missing self" strategy allows innate immunity to thresh out cells that have attempted to downregulate MHC Class I expression; a strategy exploited by malignant or virally infected cells. NK cells also critically require stimulation of activating receptors to engage their effector functions. These receptors (and their ligands) include: DNAM1 (CD155), NKG2D/C (MICA,MICB, 
RAET1E), NKp46 and FCYRIIIA (IgG/ADCC) (M. G. Morvan \& L. L. Lanier, 2016). If an NK cell is triggered through receptor ligand binding, a formidable armament can be brought to bear against the offending cell including perforin and granzymes (perforin creates pores in the membrane through which the serine protease granzymes enter), FAS ligand and TRAIL. (Maelig G. Morvan \& Lewis L. Lanier, 2016)

Mechanistically, upon conjugation, NK cells form an immunological synapse with the target cell. NK integrins, namely lymphocyte functional antigen-1 (LFA-1) stabilize this physical association. Recognition of activating ligands displayed on the target cell induces receptor signaling in the NK cell that results in a high affinity conformation change in LFA-1 (Osman, Burshtyn, \& Kane, 2007). LFA-1 binds to the intercellular adhesion molecule 1 (ICAM-1) displayed on target cells. LFA-1:ICAM-1 ligation initiates an NK signaling cascade that promotes synapse maturation and increased recruitment of LFA-1, talin-1 and F-actin to the conjugation region, also known as the cSMAC (central supra-molecular activating cluster) (Culley et al., 2009; Vyas et al., 2001). The immunological synapse is tightly regulated, and, illustrating the specific sensitivity to NK ligands, in vitro co-culture of NK cells with both autologous and susceptible target cell lines revealed that instances where a coincident triple conjugation occurred (one NK conjugated to both an autologous and a susceptible cell) a cytolytic synapse was strongly favored towards the susceptible cell, while the autologous cell was largely left alone (Vyas et al., 2001). Successful initiation of a cytolytic immune synapse results in localization of cytotoxic vesicles containing perforin and Granzyme B that are maintained in a neutralized state by electrostatic interactions with the heparin sulfate proteoglycan Serglycin (S. M. Raja, Metkar, S. S., Honing, S., Wang, B., Russin, W.A., Pipalia, N.H., Menaa, C., Beling, M., 
Cao, X., Dressel, R., Froelich, C.J., 2005; S. M. Raja et al., 2002). Serglycin has been implicated in other non-immune cells as well, with documented tumorigenic roles pertaining to inflammation and EMT (Bouris et al., 2018; Manou, Karamanos, \& Theocharis, 2020). The cytotoxic vesicles arrive at the immune synapse and are thought to exit the NK cell through permissible regions of f-actin meshwork and are exchanged into the target cells (Vyas et al., 2001).

Thus, ICAM-1 expression is a critical surface molecule mediating NK cytotoxicity. Its regulation can dictate the extent to which NK cells can effectively conduct immunosurveillance. In acute myeloid leukemia (AML), GSK3 $\beta$ activation in NK cells can render them less potent. Ex vivo treatment of human donor NK cells with a GSK3 $\beta$ inhibitor rescued cytotoxic activity in an LFA-1:ICAM-1 dependent manner. GSK3 $\beta$ inhibition not only upregulated LFA-1 expression on NK cells, but also lead to increased ICAM-1 expression on target cells in response to increased TNF $\alpha$ secretion by the NK cells. In this model, perforin and granzyme B levels only changed modestly, underscoring the significance of ICAM-1 expression in facilitating NK killing (Parameswaran et al., 2016). ICAM-1 is also upregulated in response to radiation in numerous cancer cell lines, facilitating NK killing relative to unirradiated controls. Of note, NK cells also secreted significantly higher levels of IFN- $\gamma$ when co-cultured with irradiated HL60 and T47D compared with unirradiated co-culture. (Jeong et al., 2018)

Natural Killer cells are major contributors to "natural cytotoxicity", an appreciated predictor of antitumor immunity. A large cohort study conducted over 11 years found that healthy donors whose peripheral blood lymphocytes measured higher, baseline cytotoxic activity had lower cancer incidence over time (K. Imai, Matsuyama, Miyake, Suga, \& Nakachi, 2000). Older studies conducted on patients with familial melanoma and familial breast cancer 
indicated that these patients were more likely to have lower peripheral blood cytotoxic activity against cultured target cells, implicating cytotoxic activity in cancer incidence (Hersey, Edwards, Honeyman, \& McCarthy, 1979; Strayer, Carter, \& Brodsky, 1986). Further evidence in mouse models suggest that NK cells likely influence the dynamics of primary tumor establishment. Rag/- (lacking T and B cell lineage) deficient mice were additionally depleted of NK cells via knockout of II2-receptor gamma. Rag $^{-/}$mice lacking NKs exhibited increased occurrence of methylcholanthrene initiated sarcomas: an effect attributed to lack of NK-derived IFN- $\nu$ stimulation of M1 macrophages, demonstrating a role for innate immunity during primary tumor development (O'Sullivan et al., 2012). Ablation of NK activating receptors DNAM1 $\%$ or NKp46-- causes development of tumors enriched for the respective ligands in sarcoma models (Elboim et al., 2010; Iguchi-Manaka et al., 2008). Further, voluntary exercise has been shownto increase NK infiltration and reduce tumor burden in mice through $\beta$-adrenergic activation of IL6 responsive NK cells (Pedersen et al., 2016).

NK-specific alterations in mouse tumor models have been especially informative in linking NK cells with metastasis. Deletion of $M c / 2$ in murine NK cell lineages removes NK cells from the circulation, resulting in dramatically enhanced metastases when challenged with low levels of B16-F10 melanoma cells (Sathe et al., 2014). In a colorectal carcinoma model, adoptive transfer of $\mathrm{CD} 27^{\text {low }} \mathrm{KLRG1} 1^{+} \mathrm{NK}$ cells rescued T-bet deficient mice from lung metastasis (Malaisé et al., 2014). NKs engineered to express a constitutively active TGFBR1 (inhibiting NK function in part by downregulation of receptors and loss of cytotoxicity) exhibited reduced degranulation in the presence of target cells, while NK-specific ablation of TGFBR2 led to decreased lung 
metastasis after injection of B16-F10 or RM-1 cancer cells (Viel et al., 2016). NK-mediated intravenous clearance of circulating mammary tumor cells was demonstrated in D2A1 intravenously injected BALB/C mice which had been treated with anti-asialo-GM-1 antibody, these NK ablated mice exhibited 5.5x increased lung-localized D2A1 cells. Intriguingly, this model also showed that mice with a concurrent 4T1 subcutaneously implanted mammary tumor (which substantially increases systemic neutrophil numbers) mimicked the increased metastatic parameter seen in NK-depleted mice, suggesting that tumor-influenced neutrophil dynamics modulated NK activity (Spiegel et al., 2016). Similarly, utilizing NK1.1 antibody in a C56BL/6 mouse model lead to a 7x increase in lung-localized B16-F10 intravenously injected melanoma cells (Spiegel et al., 2016). The literature supports the ability of Natural Killer cells to inhibit metastasis, and influence primary tumor development through cytolytic effector functions, but also through interaction or synergy with other immune cells.

EMT has been investigated as a possible mechanism conferring resistance to immune effector-mediated cytotoxicity. Due to the gradient, non-binary nature of EMT, as well as the multiple mechanisms of immune effector cell killing, numerous avenues of EMT-derived resistance and sensitivity have been reported (Lopez-Soto et al., 2013; Terry, Buart, et al., 2017). A NSCLC primary cell line model found that that subpopulations of cells that underwent EMT in response to hypoxia were more resistant to both cytotoxic T lymphocyte (CTL)- and NK92-mediated killing relative to cells that remained epithelial under hypoxia. Although conjugation remained similar between the epi./mes. subsets, the mesenchymal cells exhibited marks of reduced immunological synapse function. Sensitivity was rescued by siRNA's targeting EMT transcription factors Zeb1, Zeb2, Snai1 and Snai2, or pharmacologic inhibition of 
mesenchymal cell TGF- $\beta$ production (Terry, Buart, et al., 2017). Mcf7 breast carcinoma cells induced to an EMT state via TNF- $\alpha$ exposure (Mcf7-2101) or ectopic Snail (Mcf7-SNAI1) cells exhibited increased resistance to CTL lysis. This resistance was linked with EMT-induced autophagy, as knockdown of beclin1 restored CTL lysis. (Akalay et al., 2013). Supporting a role for autophagy in NK sensitivity, NSCLC models, independent of EMT, demonstrated that inhibiting autophagy using RocA (inhibiting ULK1 expression among other things) caused a synergistic effect to promote NK killing in an in vivo mouse tumor model. Autophagy inhibition led to increased Granzyme B concentration in target cells (Yao et al., 2018). EMT'd derivatives of MCF7, as well as MDA-MB-231 cells exhibited significantly upregulated immunosuppressive programmed death-ligand 1 (PD-L1) expression, attributed to the EMT transcription factor Zeb1. Increased resistance to CTL lysis was attributed to PD-L1 expression, an effect that was independent of TGF- $\beta$ expression in this model (Noman et al., 2017). As described above, NKtarget conjugation and synaptogenesis requires concerted and sequential cytoskeletal arrangement and interaction. It is likely that the resultant altered cytoskeletal arrangement after EMT would augment NK conjugation and synapse formation to greater or lesser degrees (Terry, Savagner, et al., 2017). The ability of resistant breast cancer cell lines to effectively remodel actin at specific focal points enabled resistance to NK-mediated cytotoxicity in a CDC42 or N-WASP dependent manner (Al Absi et al., 2018)

Based upon these observations, it seemed plausible that Grainyhead-like 2 could play a role in maintaining proper sensitivity to anti-cancer immunity by inhibiting EMT. The central hypothesis of this chapter is as follows; through promotion of an epithelial default state, Grainyhead-like 2 will promote sensitivity to Natural Killer cell cytotoxicity. We employed 
various cell culture models to test this hypothesis. We chose multiple cell lines that could be induced to exhibit either a mesenchymal or epithelial state through various means, including knockout or overexpression of GRHL2 (See Cell Lines Diagram in Material and Methods). The stable ectopic expression of GRHL2 in Ras-transformed human breast epithelial cells (Mcf10aneoT) that had been induced to undergo stable EMT after transient addition of TGF- $\beta$, the mesenchymal human fibrosarcoma cells (HT1080), and human mammary epithelial cells (HMLE) that can be selected for a CD44hi/CD24lo mesenchymal subpopulation (MSP). We also utilized cell lines induced to undergo an MET by alternative means; expression of adenoviral protein E1a, which inhibits p300 and induces MET (Frisch, 1994, 2001), and activation of the microRNA miR200C, which has also been noted to induce MET and repress immunosuppression (Howe, Cochrane, \& Richer; Rogers et al., 2018). We explore sensitization of these cell lines to NK92mi cytotoxic attack, identify candidate molecules dictating sensitivity/resistance, and investigate GRHL2's epigenetic influence on NK sensitivity. This research opens up exciting prospects implicating GRHL2 as an important mediator of the epithelial immune setpoint. Upcoming data in Chapter II will explore the breadth of GRHL2's control of interferon signaling. These experiments could help to elucidate fundamental principles pertinent to the epithelial versus mesenchymal state as it impacts innate immunity and interferon signaling in anti-viral and anti-tumor immunity. To conclude, our data supports the hypothesis that Grainyhead-like 2 safeguards the subordinate relationship of epithelial cells to anti-tumor immunity: a relationship that is frequently manipulated and deranged during tumor progression. 


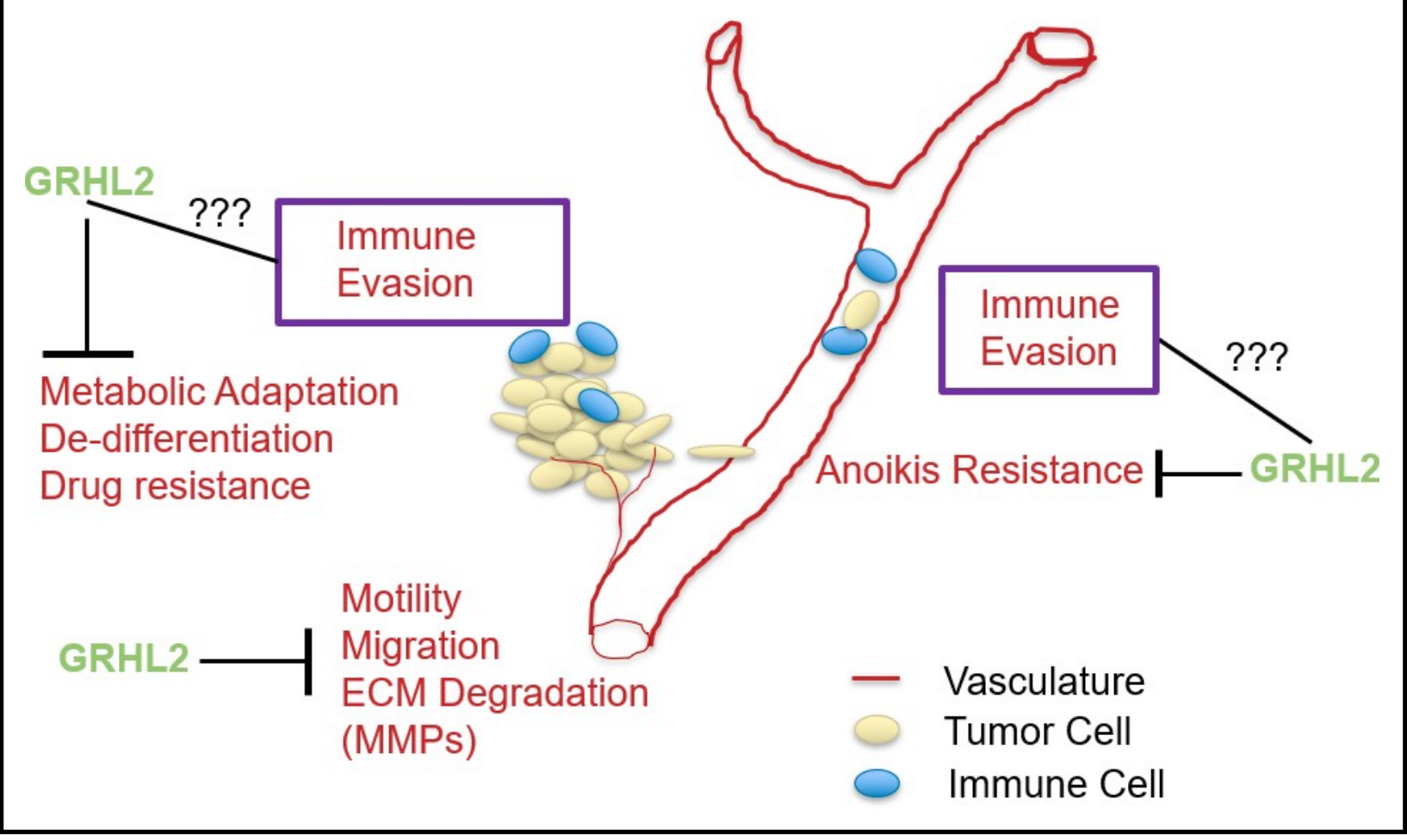

Diagram 2: The Significance of GRHL2 for Tumor Biology. Idea for figure adapted from (Sulaiman, Ab Mutalib, \& Jamal, 2016), words and drawing inserted by I. MacFawn.

\section{Chapter I: Results}

\section{I.a. GRHL2 sensitizes cells to NK92 cytotoxicity}

In order to test the effect of the epithelial state on sensitivity to NK attack, we reverted mesenchymal cell lines to an epithelial state through induction of GRHL2, miR200C, or E1a protein. We retrovirally expressed GRHL2 in a CD44 ${ }^{\text {hi }} \mathrm{CD} 24^{\text {lo }}$-sorted mesenchymal subpopulation (MSP) of human mammary epithelial (HMLE) cells, as well as Mcf10aneoT cells that had undergone EMT in response to TGF- $\beta$, and HT1080 fibrosarcoma cells (Cieply et al., 
2012; Farris et al., 2016; Pifer et al., 2016). Additionally, we expressed an inducible miR200C microRNA in BT549 breast cancer cells (Howe et al.; Rogers et al., 2018), and adenovirus E1a protein in HT1080 cells (Frisch, 1994, 2001; Pifer et al., 2016) to induce MET. The isogenic epithelial and mesenchymal variants of each cell line served as target cells, while the immortalized human NK92-MI cells were used as the immune effector cell. Using NK cells as our immune effector cell was appropriate because of their documented, efficient anti-metastatic function, and, in contrast to CD8+ T-cells, specifically target cells that lack MHC Class I surface expression. Additionally, NK-92 Ml cells, in contrast to endogenous NKs, are no longer inhibited by MHC Class I expression due to deletion of Killer cell immunoglobulin receptor (KIR), and have an IL-2 transgene (Klingemann, Boissel, \& Toneguzzo, 2016). Because of these subtleties, the NK92-MI cell line allow us to use one broadly applicable effector population to test sensitivity to cytotoxic killing across a variety of non-syngeneic target cells.

Our preferred method of assessing viability during NK assays was through stable ectopic expression of a "Nano-luciferase" construct in target cells, which allows for quantification of cell death as a luminescence signal (Rossignol, Bonnaudet, Clemenceau, Vie, \& Bretaudeau, 2017). Some assays were also conducted using a dual staining method, or using PrestoBlue viability reagent. We observed markedly increased sensitivity to NK attack in our epithelial derivatives compared to mesenchymal cells when adherent cells were co-cultured with NK cells over a time course ranging from 4-24hrs (Fig 1.a). Normalized luminescence was used as a measure of Nano-luc released into supernatant resulting from membrane degradation in dying target cells. NK assays conducted in suspension, with non-adherent target cells, perhaps better mimicking cellular interactions during metastatic dissemination, also yielded similar results (Fig 
1.b). To expand upon this finding, we also tested HT1080 fibrosarcoma and BT549 breast cancer cells induced to undergo MET by alternative pathways and found that they, too, exhibited increased sensitivity to NK attack when we expressed adenoviral E1a (inhibits HAT p300) and miR200C (MET inducer), respectively (Frisch, 2001; Howe et al.). These data support our hypothesis that sensitivity to Natural Killer cells is a feature of the epithelial state, and is consequentially altered by EMT.
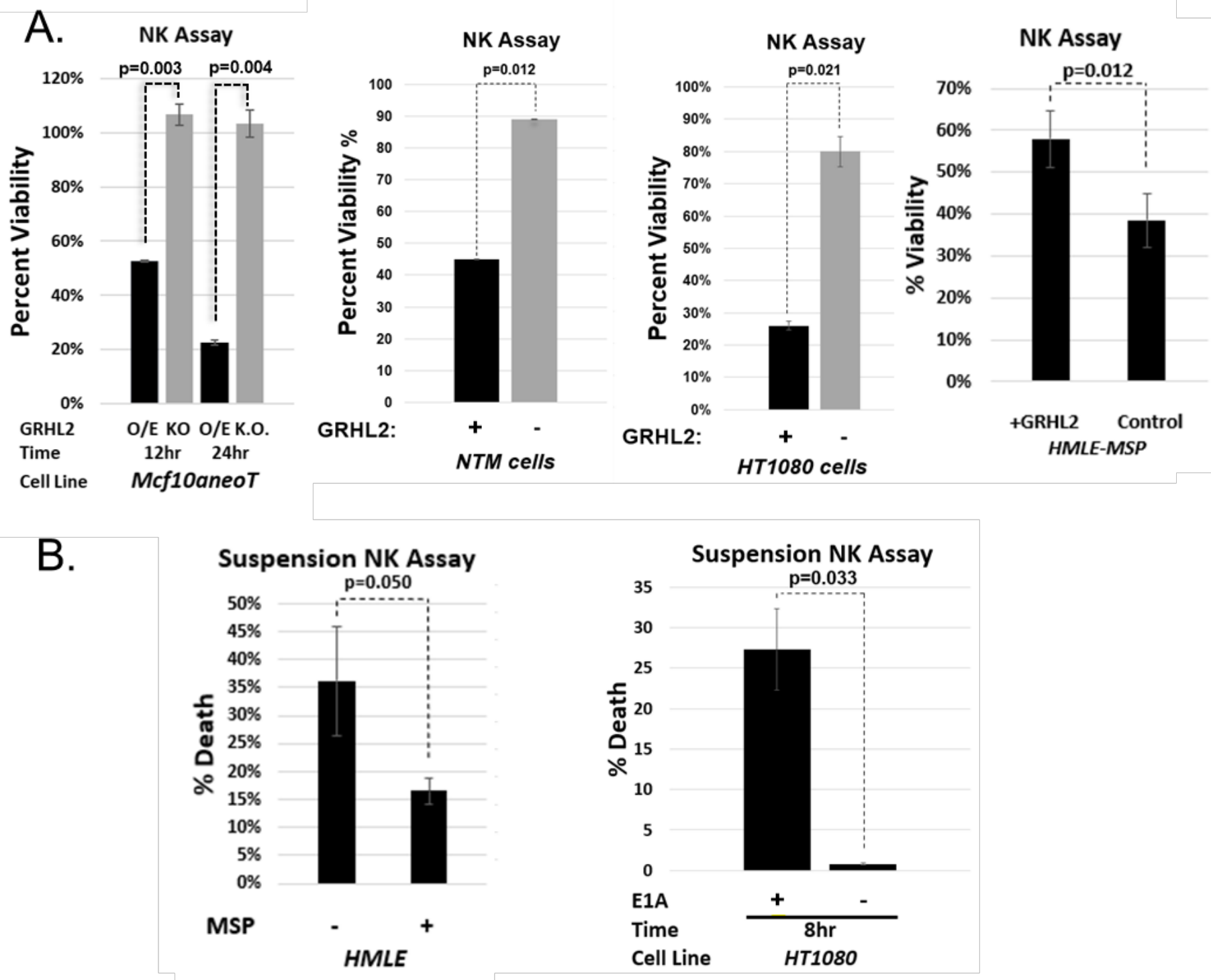


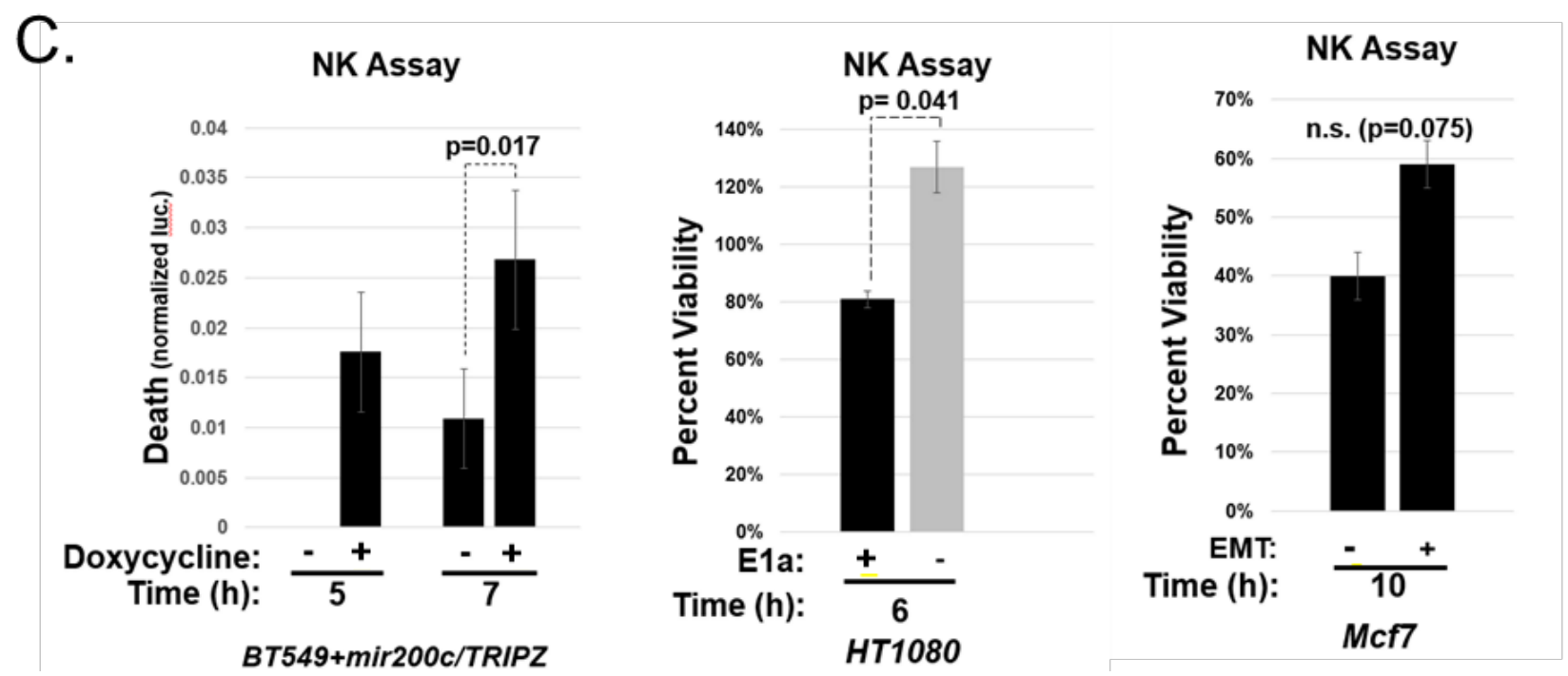

Fig 1. GRHL2 sensitizes cells to NK92 cytotoxicity. A.) Mcf10aneoT+GRHL2/sgGRHL2, Mcf10aneoT+TGF-6+GRHL2/mxs ("NTM"), HT1080+GRHL2/mxs, and HMLE/HMLE-MSP cells were plated at equal density in at least biological duplicate. When cells were $~ 70 \%$ confluent, NK92mi cells were added at 2:1-5:1 ratios and incubated until significant death was observed. B.) Target cells were suspended and mixed with NK92mi cells and resuspended periodically throughout the Suspension NK assay. C.) Additional methods of MET were utilized: i.) doxycycline inducible miR200C induced MET, dox was withdrawn prior to NK assay, ii.) a stable E1a adenoviral construct induces MET in HT1080, and iii.) an EMT'd derivative of Mcf7. \%Viability was measured as [sample (X) lum. Signal] / [avg NO NK replicates lum. signal]. Pvalues calculated using Student's Two-tailed T test.B.) Suspension NK assays involved trypsinizing, counting and suspending target cells at equal densities in microfuge tubes. NK92mi cells were added at 3:1 ratio. At timepoints, an aliquot was taken from the microfuge tube, mixed with Nano-glo complete reagent and luminescence value was read on platereader. These samples were blanked against a NO NK anoikis value, then normalized to a lysed NO NK sample 
to calculate \% Death. Assay Details: A1: Duplicate, 12/24hr time points. A2: Duplicate A3:

Duplicate, 24hr timepoint. A4: Quadruplicate. B1: Triplicate. B2: Duplicate. C1: Quadruplicate.

C3: Duplicate.

I.b. The effect of Inhibitory/Activating NK Ligand

\section{Expression and Fadd knockdown on NK Killing}

We examined multiple hypotheses that could

account for the increased sensitivity of epithelial cells to

NK attack. We checked a previously generated RNAseq

dataset comparing HMLE derivaties (Farris et al., 2016) for

classical activating or inhibiting NK cell ligands, as well as

adhesion molecules. We did not find any dramatic

differential expression of NK ligands or receptors between

mesenchymal and epithelial cells, though some were

altered over 2-fold (Table 1). NK cells kill target cells via

two direct mechanisms: delivery of perforin/granzyme B

through an immune synapse, and/or cell surface

expression of death ligands Fas and TRAIL. To distinguish

between the method of killing in our system, we used an

siRNA against Fadd; a critical adaptor protein that relays

the death receptor ligand-binding signal to pro-caspases 8
Table 1.

HMLE-MSP versus HHLE-MSP+GRHL2

NK Activating/lnhibitory Ligands

Activators

RAET1L

CEACAM1

ULBP1

RAET1E

BAG6

CD112 (PVRL2, Nectin2)

CD155 (PVR)

CD96

IL15

MICB

IFNGR1

MICA

RAET1K

RAET1G

CD70

$\mathrm{CD} 226$

CD137/CD36

IFNGR2

CD80

CD86

LL2

Inhibitors

LGALS9

HLA-B

LGALS3

HLA-A

HLA-G

HLA-E

CD48

CLEC2D(LLT1)

serglycin

SPARC

Adhesion molecules

$\mathrm{CD} 48$

CD58

ICAM1

ICAM2

NCAM1

HSPG2
(+)GRHL2 Fold Change

NE

NE

2. 715743812

2.601826182

NE

1.377718929

1.347843426

NE

1.533120226

NE

1.218904975

$-1.044377401$

NE

$-1.380480439$

$-1.835080565$

NE

NE

$-3.876863417$

NE

NE

NIE

NE

1.959455354

1.496208253

NE

NE

NE

$-1.079573739$

$-9.59173851$

$-11.97982993$

\begin{tabular}{c} 
NE \\
\hline-1.394221581 \\
\hline 1.977037416 \\
\hline$N E$ \\
NE \\
\hline-4.578150689 \\
\hline
\end{tabular}

and 10, thereby initiating apoptosis. We knocked down Fadd in Mcf7, and Mcf10a cells, verified knockdown via western blot (Fig 2a), then conducted NK assays on these cell lines. Knockdown 
of Fadd did not rescue cells from NK-mediated death (Fig 2b), suggesting that in our system, the NK cells were killing through a different mechanism, likely perforin/granzyme B delivery through the immune synapse. This insight allowed us to target a narrower range of possible molecules and mechanisms involved in epithelial sensitization to NK attack in our system.

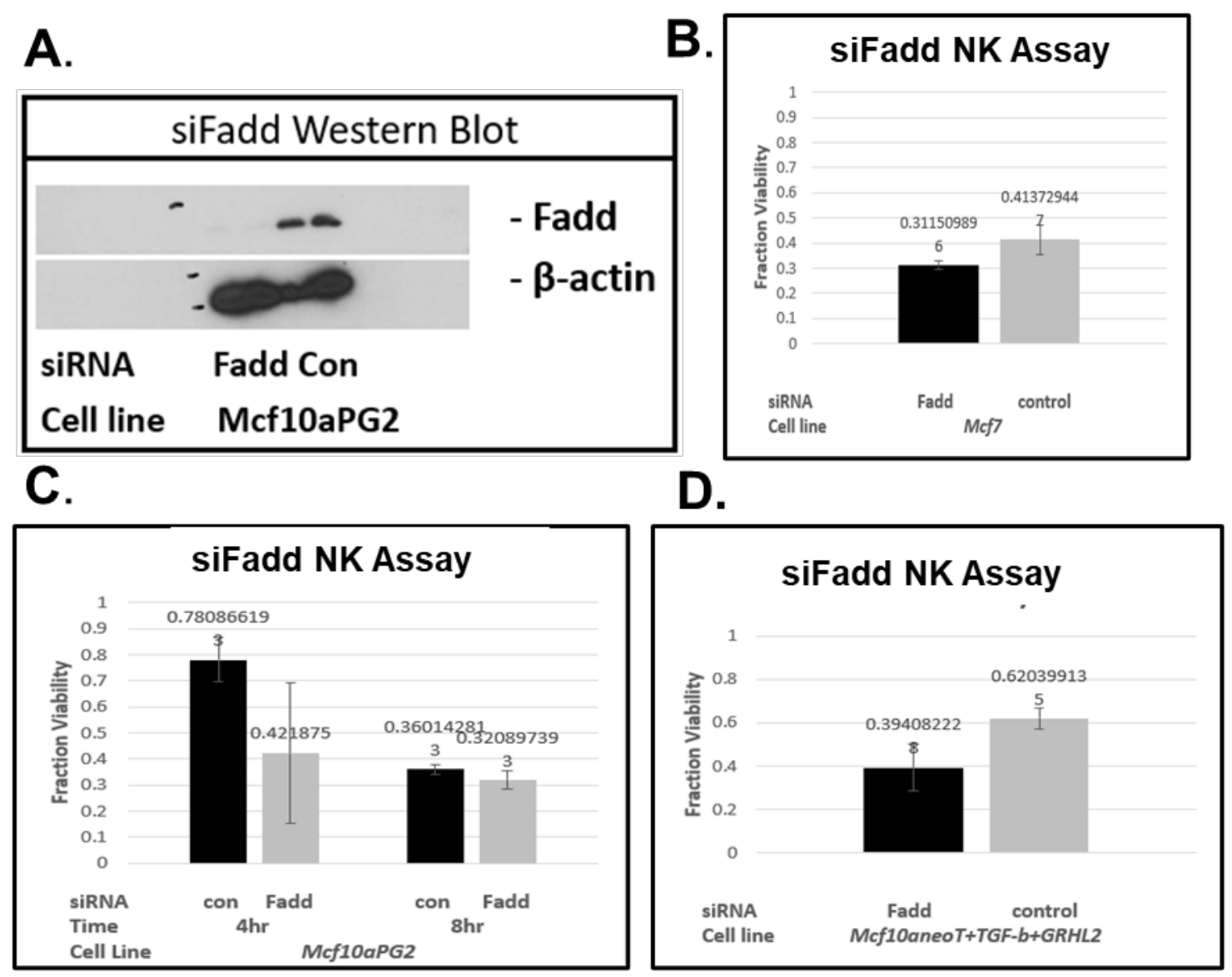

Figure 2. GRHL2's promotion of NK sensitivity is independent of death receptor signaling. Fadd was knocked out by siRNA in Mcf10a and Mcf7 cells. A.) Western blot indicates siRNA mediated knockdown of Fadd. B.-D.) siFadd Mcf7 and Mcf10a cell lines did not exhibit increased resistance to NK92mi killing in NK assays. NK assays conducted in duplicate. 


\section{I.c. The effect of epithelial versus mesenchymal ECM on NK92 killing.}

One major difference between epithelial and EMT'd cells is the amount and type of factors they secrete to develop extracellular matrix (ECM). Cells have a close relationship with underlying matrix and attach to these deposited factors through integrins, influencing intracellular signaling. Matrix composition or stiffness can change cytoskeletal dynamics and even induce EMT (Wei et al., 2015). We tested the effects of the specific matrix the cells were plated on by conducting matrix switch Granzyme B and NK assays. We separately plated epithelial and mesenchymal derivatives of a cell line and allowed the cells to grow to confluence. Using ammonium hydroxide $\left(\mathrm{NH}_{3} \mathrm{O}_{3}\right)$, we lysed the cells but retained their secreted matrix. We then plated sub-confluent numbers of epithelial cells on the mesenchymal matrix and vice versa. Interestingly, we found that in some cases, the epithelial matrix sensitized the mesenchymal cell line to Natural killer cell cytotoxicity (Fig $3 a, b, c)$. This could imply that matrix components may regulate cell morphology and architecture, thereby effecting conjugation of immune effector cells to target cells. Despite numerous experiments and repeats, this effect was too often variable, despite reaching statistical significance in multiple experiments. This phenomenon warrants further investigation, but we decided to prioritize other, more tractable explanations for NK sensitization. 
A.

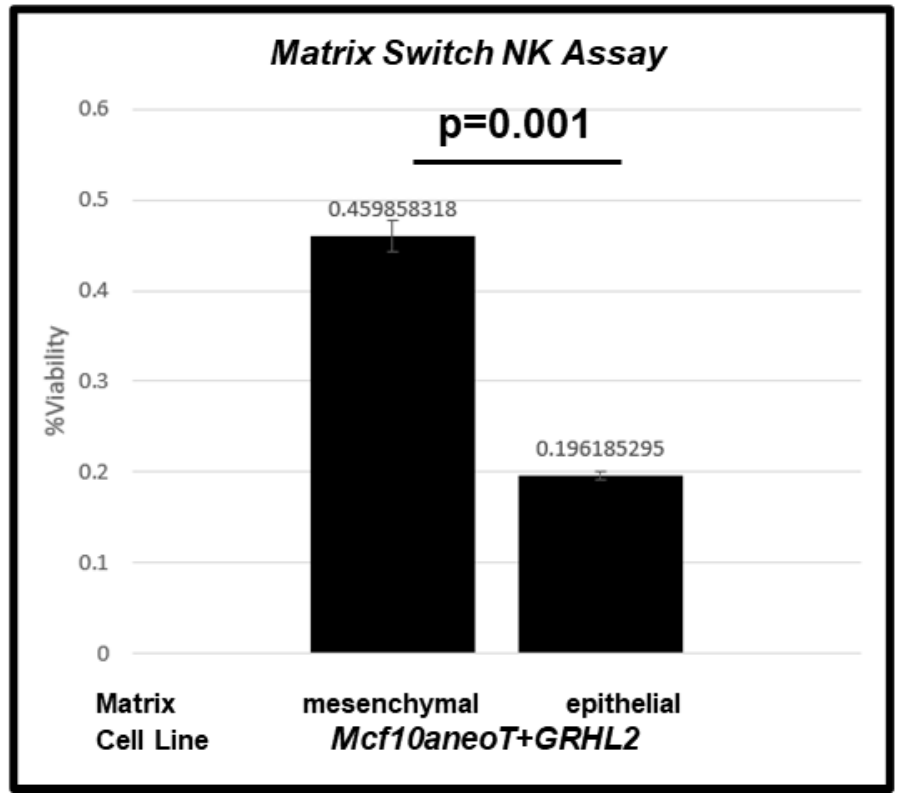

B.

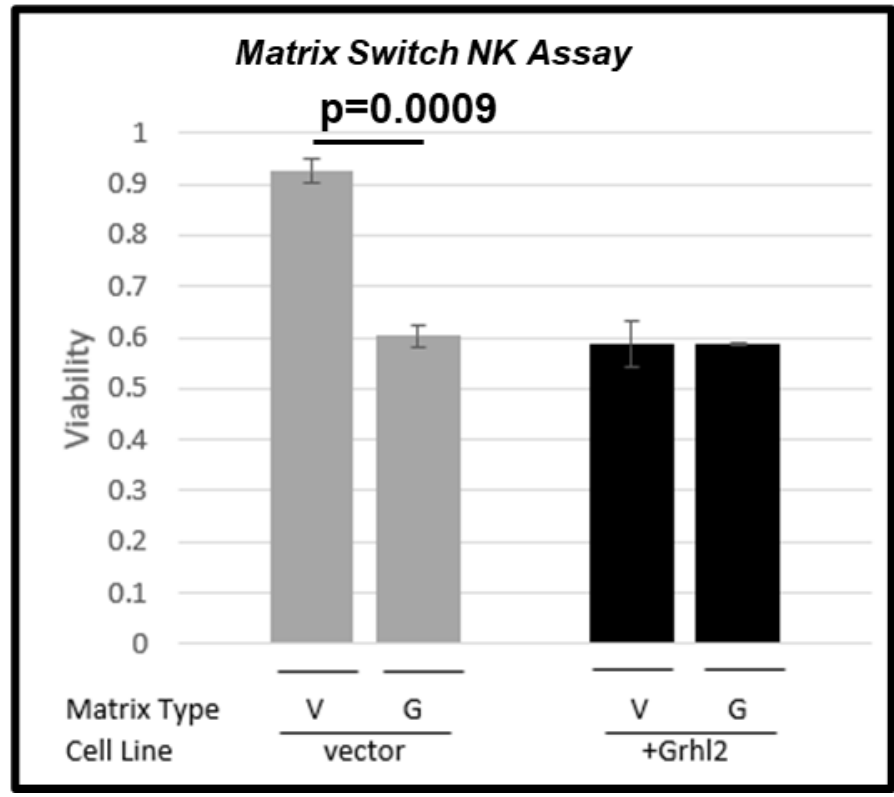

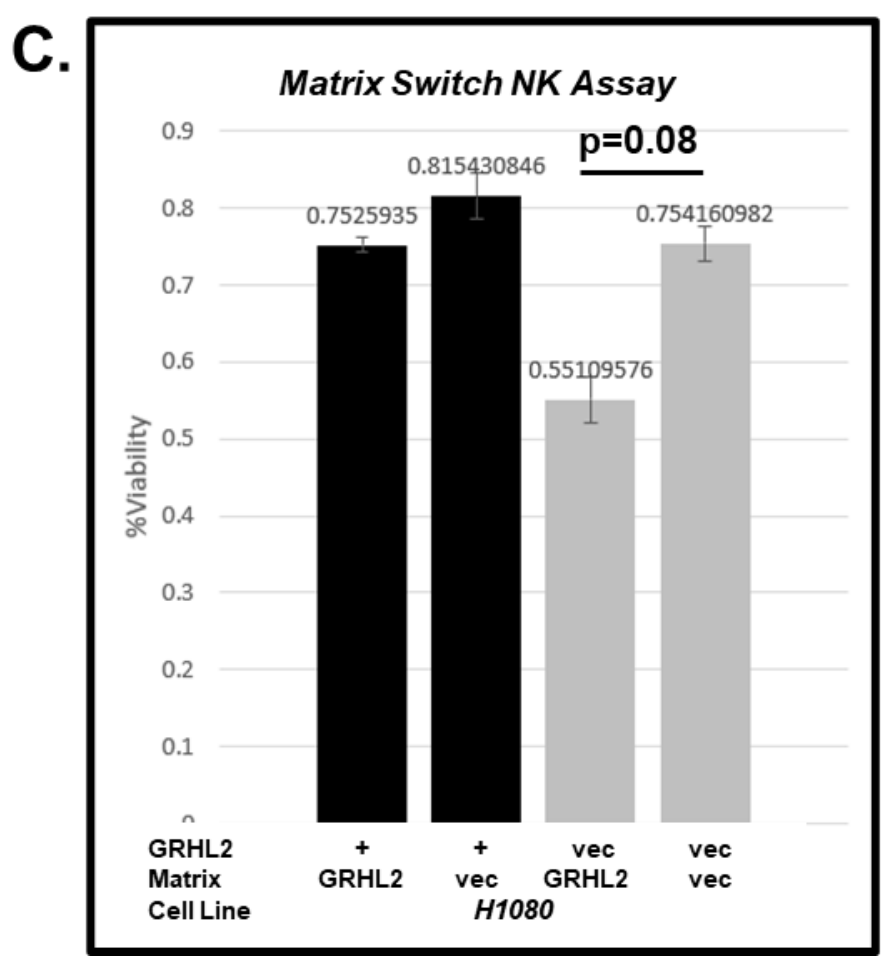

Figure 3. Matrix effects on NK sensitivity. A.) Mcf10aneoT+TGF-b+GRHL2/mxs were plated at equal density in duplicate or B.) triplicate. Cells were grown to confluence, lysed in 
ammonium hydroxide, then wells were stringently washed and the opposite state cell (mes./epi.) was plated at equal densities onto the opposing matrix type. After attachment, NK92mi cells or C.) HT1080 (+GRHL2) or (+control vec) were plated in duplicate, as above. NK cells were added and viability was calculated after significant death was apparent.

\section{1.c. GRHL2 suppresses expression of the proteoglycan Serglycin}

We also explored the role of the chondroitin sulfate proteoglycan Serglycin (SRGN).

Serglycin has been implicated in tumor malignancy and the establishment of a proinflammatory microenvironment (Bouris et al., 2018; Korpetinou et al., 2014). Interestingly, SRGN also contains a protease resistant core structure which is utilized by immune cells, notably NKs, to neutralize proteolytic enzymes within secretory vesicles containing perforin and Granzyme B (S. M. Raja, Metkar, S. S., Honing, S., Wang, B., Russin, W.A., Pipalia, N.H., Menaa, C., Beling, M., Cao, X., Dressel, R., Froelich, C.J., 2005). In Mcf10aneoT and HT1080, mRNA measured by qRT-PCR indicated SRGN is strongly repressed by GRHL2 (Fig. 4 a,b). RNAseq datasets agreed with these findings; Serglycin is upregulated 205-fold upon GRHL2 knockout in a Mcf10aneoT (Fig. 4, c). We thought that Serglycin expression may be a means for EMT'd cells to neutralize the cytotoxic molecules of immune effector cells (perforin, granzyme B). We packaged a secreted serglycin construct into pMXS-ires-Blast vector and retrovirally overexpressed it in epithelial Mcf10aneoT+GRHL2, Mcf10a and HT1080+GRHL2 cells. We also harvested conditioned media from mesenchymal cells and conducted NK assays in the presence of secreted media, after verifying the presence of SRGN by Western Blot. Despite the high expression levels of this protective proteoglycan, we did not see significant protective effect of SRGN overexpression or conditioned media as measured by cell viability during NK attack or 
treatment with recombinant Granzyme B and streptolysin (a pore-forming enzyme that functions analogously to perforin). Mcf10aneoT+TGF-b+GRHL2 epithelial cells exhibited a nonstatistically significant effect upon SRGN overexpression, while HT1080+GRHL2 cells showed a large, but not statistically significant protective effect of +SRGN C.M. when measured for sensitivity to Granzyme B (Fig 4. c). It is possible that the disproportionate expression of Serglycin between epithelial and mesenchymal cells in our models contributes to other protumorigenic processes, but it appears to have a modest or negligible impact on direct immune cell-mediated killing in our models.

A.
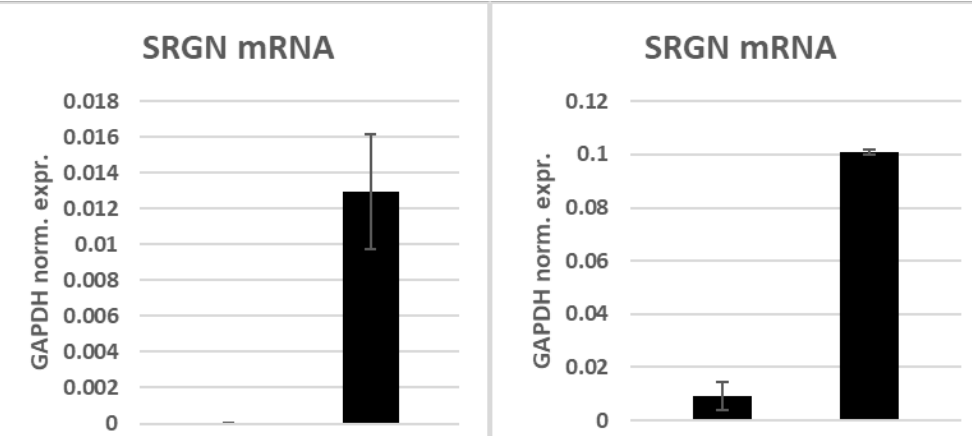

$$
\begin{array}{cc|ccc}
\text { GRHL2 } & \text { O/E } \\
& \text { Mcf10aneoT } & \text { K.O. } & \text { GRHL2 } & \text { O/E } \\
& & & \text { HT1080 }
\end{array}
$$

\begin{tabular}{|c|c|c|c|c|c|c|}
\hline \multicolumn{7}{|c|}{ Mcf10aneoT RNAseq (FPKM) } \\
\hline symbol & WT \#1 & WT \#2 & WT \#3 & KO \#1 & KO \#2 & KO \#3 \\
\hline & 1.02292 & 0.98986 & 1.21806 & 213.107 & 215.249 & 235.198 \\
\hline SRGN & 8 & 2 & 1 & 7 & 8 & 2 \\
\hline
\end{tabular}

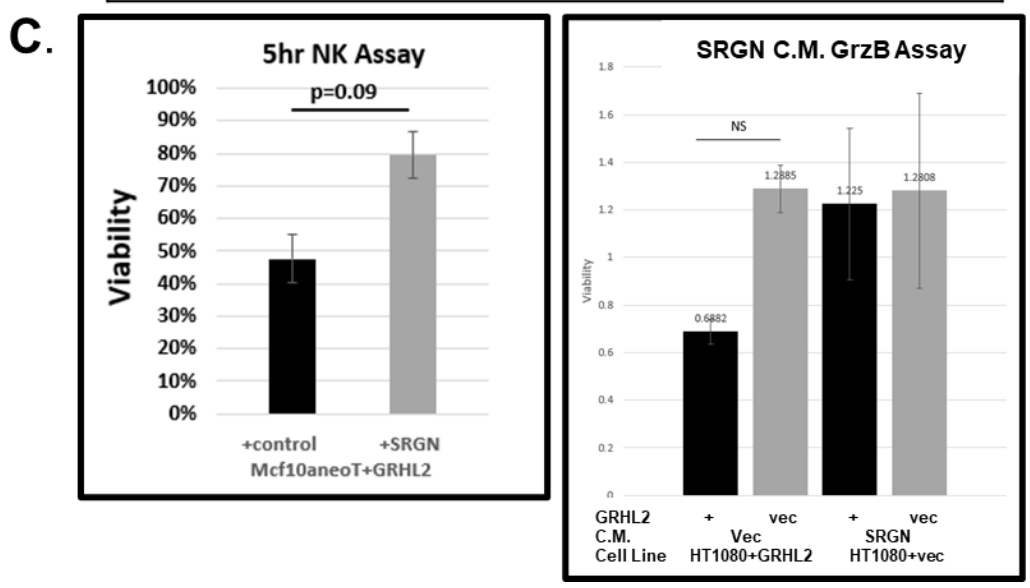


Figure 4. GRHL2 represses Serglycin expression. A.) qPCR analysis of Serglycin mRNA levels in Mcf10aneoT+TGF-6 +GRHL2, Mcf10aneoT+TGF-B+sgGRHL2 and HT1080, HT1080+GRHL2 normalized to GAPDH. B.) RNAseq mRNA expression levels of Serglycin in Mcf10aneoT, Mcf10aneoT+sgGRHL2. C.) NK killing assays were conducted on Mcf10aneoT+GRHL2 cells with or without stable overexpression of SRGN, assays conducted in duplicate. Similarly, Granzyme B sensitivity was measured on HT1080 cells (+/-GRHL2) in the presence or absence of +SRGN conditioned media, assays conducted in triplicate. Statistical analysis was conducted using a Student's Two Tailed T test.

\section{I.d. GRHL2 suppresses autophagy in HT1080 cells}

Reports suggest that upregulation of autophagy can modulate target cell sensitivity to immune attack (Akalay et al., 2013). Breast cancer cells that upregulate autophagy become more sensitive to NK-mediated killing through sequestration of anti-apoptotic proteins in the lysosome (Akalay et al., 2013) (Yao et al., 2018) . Conversely, Renal cell carcinoma cells that upregulated autophagy upon interaction with NK cells displayed better survival and lower intracellular Granzyme B levels (Messai et al., 2014). We hypothesized that differential autophagy could be a mechanism for resistance to NK attack. We expressed a tandem GFP/RFPLCIII (tfLCIII-GFP) vector in our HT1080/HT1080+GRHL2 cells. Cells were first Glucose-starved in HBSS for $2 \mathrm{hrs}$, then incubated in $100 \mathrm{nM}$ Bafilomycin for $2 \mathrm{hrs}$. Cells were fixed and autophagic flux was calculated by subtracting the LCIII punctae in (-Baf) cells from the average LCIII punctae in (+Baf) cells. Because Bafilomycin halts fusion of the autophagosome and lysosome, the 
difference between +/- Bafilomycin represents autophagic flux. We found that expression of GRHL2 significantly reduced autophagic flux in HT1080 cells (Fig 5. a,b). However, successful knockdown of Beclin, a key autophagy protein, by siRNA in HT1080 (HTM2) and Mcf10aneoT (NTM2) cells, caused statistically non-significant changes to NK-sensitivity (Fig 5. c,d,e,f). Our data suggests that EMT modulation can have effects on autophagy rates, but in our models, this did not seem to contribute significantly to NK sensitivity. 

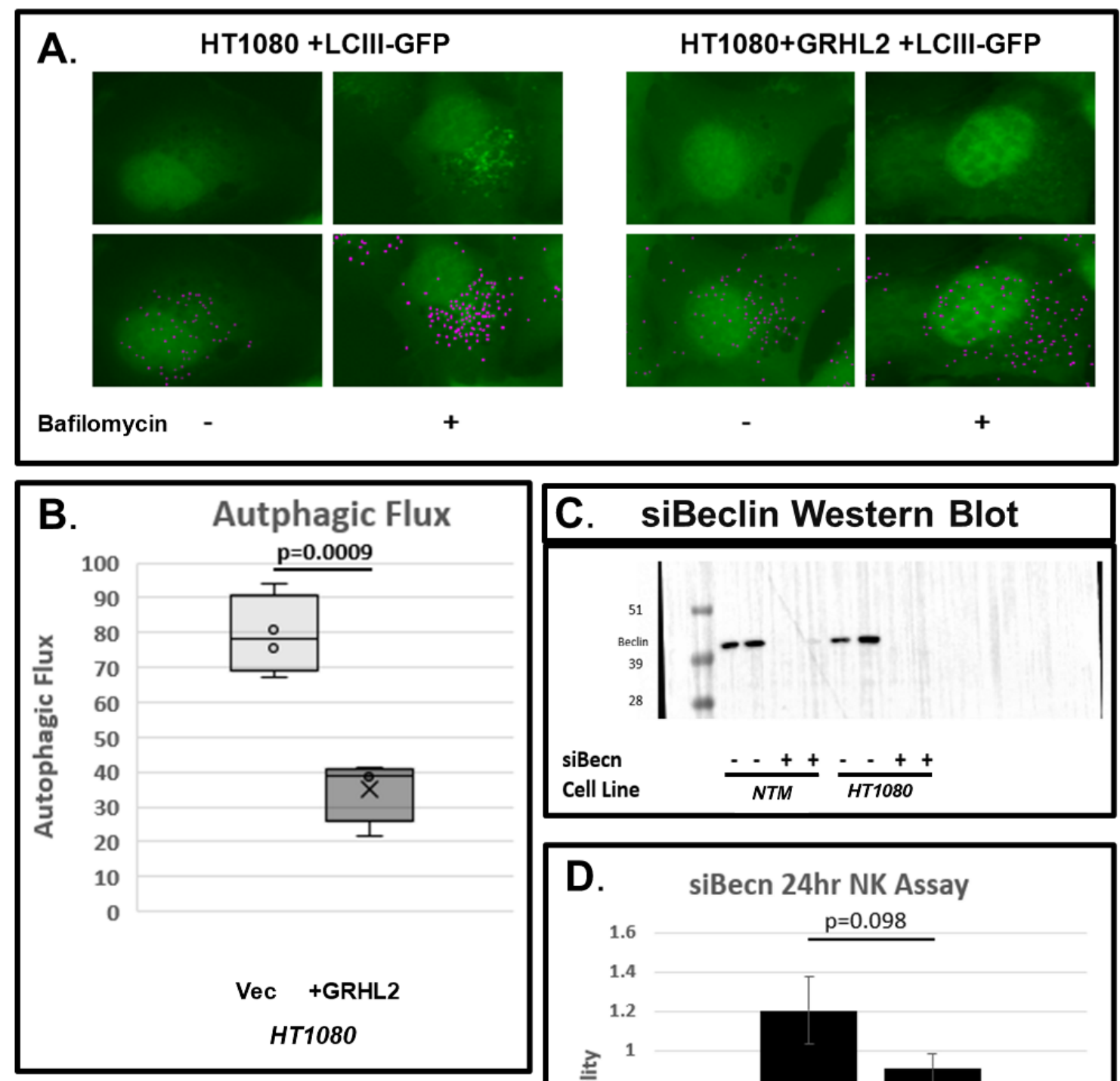

E. siBecn 10hr NK Viability

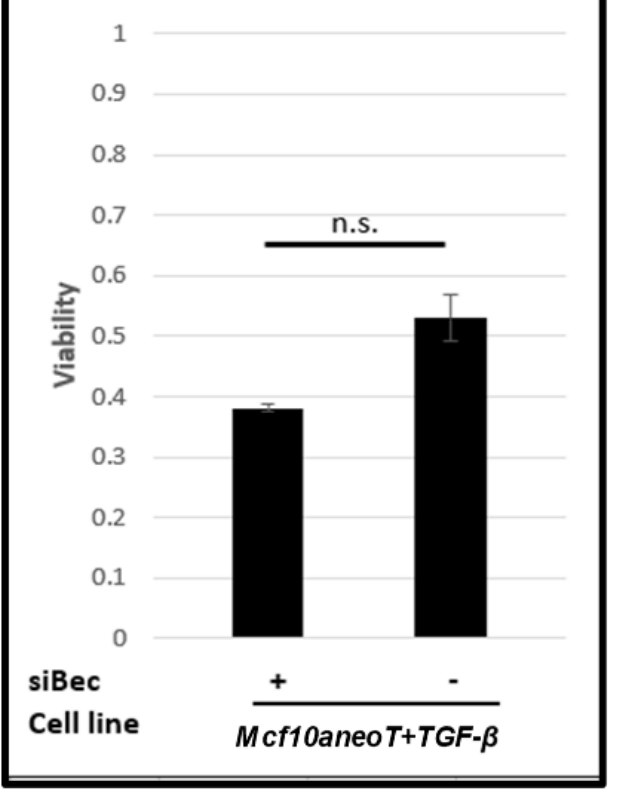

C. siBeclin Western Blot
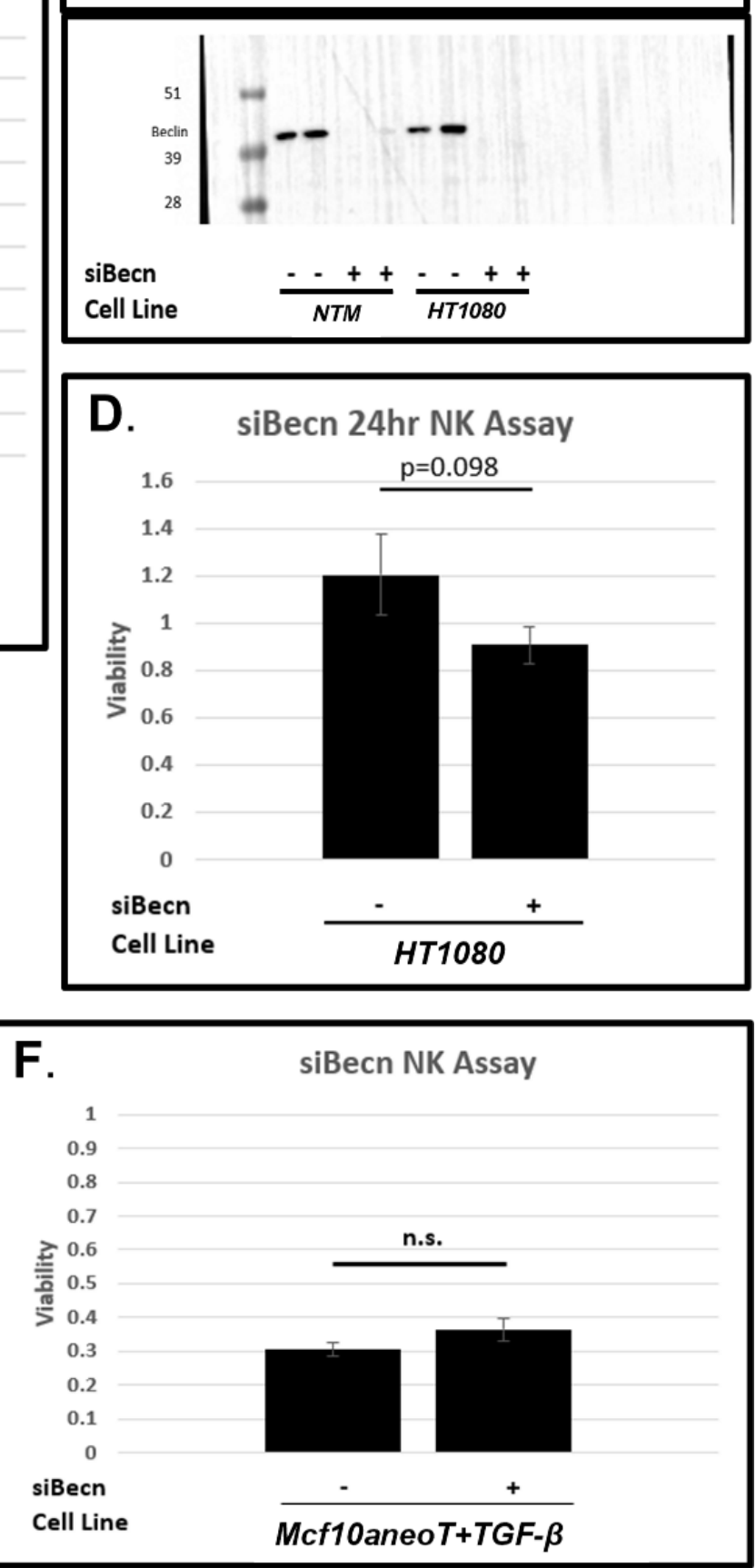
Figure 5. GRHL2 regulates autophagy. A.) After expression of a fluorescent LCIII plasmid (tcfLCIIIGFP), HT1080+vec ("HTM2") or HT1080+GRHL2 ("HTG2") cells or Mcf10aneoT+TGF-b+vec ("NTM") or Mcf10aneoT+TGF-6+GRHL2 ("NTG2") were glucose starved in HBSS for 2hrs, then incubated in 100nM Bafilomycin for $2 \mathrm{hrs}$. Cells were fixed, then number of punctae in (-) Bafilo was subtracted from punctae in (+) bafilo. to calculate autophagic flux on a fluorescence microscope. B.) Quantification of images. C.) To inhibit autophagy, we transfected Beclin siRNA to knockdown protein levels in NTM and HTM cells. D-F.) NK assays were conducted on Beclin knockdown cells as described previously. Cells were plated in a minimum of biological duplicate. Statistical analysis was conducted using Student's Two Tailed T test. Assay details: 5D: Triplicate. 5E: duplicate. 5F: Triplicate.

\section{I.e GRHL2 promotes NK92/target cell conjugation through upregulation of ICAM-1}

Differences in NK assay dynamics, visually apparent under low power magnification, suggested that immune cell synaptogenesis could be augmented by epithelial state. The immune synapse is an essential part of direct NK cell-mediated cytotoxicity. NK cells approach, and physically conjugate with target cells. If the balance of activating versus inhibitory ligands is permissive, they initiate a complex polarization and rearrangement of the cell membrane, termed the "immune synapse", towards the target cell, through which they release the contents of granular cytotoxic vesicles. These granules include Perforin, which creates small pores in the target cell membrane allowing other granules, like Granzyme A and B: serine proteases involved in initiator and executioner caspase activation and mitochondrial ROS 
deregulation and DNA damage induction, respectively, to pass into the target cell (Lieberman, 2010) (Afonina, Cullen, \& Martin, 2010). Upon incubation with NK cells, we noticed that epithelial cells seemed to attract more NK cells, causing them to cluster and stick to the target cells. These NK cells remained bound to epithelial cells through multiple washings of the cells, suggesting strong and stable physical connection.

To quantify the conjugation of NK92 to target cells we separately stained target cells with CMFDA and NK92mi cells with CMPTX allowing fluorescent detection of different cell populations. We washed the populations of cells numerous times, then conducted NK assays using the stained cells. At timepoints, we gently washed the wells with sPBS to removed unconjugated NK cells, then fixed the cells with paraformaldehyde and imaged on an epifluorescence microscope. The ratio of NK to target cell (NK conjugation) was elevated in cells expressing GRHL2 compared with the mesenchymal derivative in both Mcf10aneoT+TGFb+GRHL2 versus Mcf10aneoT+TGF-b and HMLE versus HMLE-MSP (Fig 6. a-c)

The conjugation efficiency prompted us to re-assess the expression of adhesion molecules on target cells. Intercellular adhesion molecule 1 (ICAM-1) mRNA was upregulated by GRHL2 in HMLE, HT1080 and Mcf10aneoT (Fig 6. d). ICAM-1 is an interferon stimulated gene (ISG), and is strongly upregulated by the NK- and T cell-secreted cytokine interferon gamma (IFN- ) (M. L. Dustin, Rothlein, Bhan, Dinarello, \& Springer, 2011) (M. G. Morvan \& L. L. Lanier, 2016). ICAM-1 expression is an important mediator of the antiviral and anti-tumor immune response; its cell surface expression facilitates adhesion and conjugation of immune effector cells via formation of the immunological synapse through interaction with LFA-1 on immune cells (Michael L. Dustin, 2014). Western blot analysis supported the preferential expression of 
ICAM-1 in cells expressing GRHL2 (Fig. 6 e,f). Further, we found that cells lacking GRHL2 exhibited severely limited ICAM-1 protein expression in response to IFN- $\gamma$ stimulation in vitro (Fig. 6 e,f). To test the dependence of NK sensitivity on ICAM-1 expression, we used an ICAM1 monoclonal antibody to block ICAM-1 on the surface of target cells. HMLE and HT1080+GRHL2 epithelial cells were pre-incubated with the $\alpha$-ICAM-1 antibody, washed thoroughly to remove excess antibody, then subjected to NK92mi cells. Pre-blocking epithelial cells with ICAM-1 antibody dramatically reduced NK92mi-mediated killing (Fig 6. g). We propose that ICAM-1 is maintained in epithelial cells by GRHL2 under basal conditions, and that GRHL2 could be an important regulator of the interferon response because treatment with IFN- $\gamma$ for up to $16 \mathrm{hrs}$ had a comparatively limited effect on ICAM-1 mRNA and protein in the absence of GRHL2. 

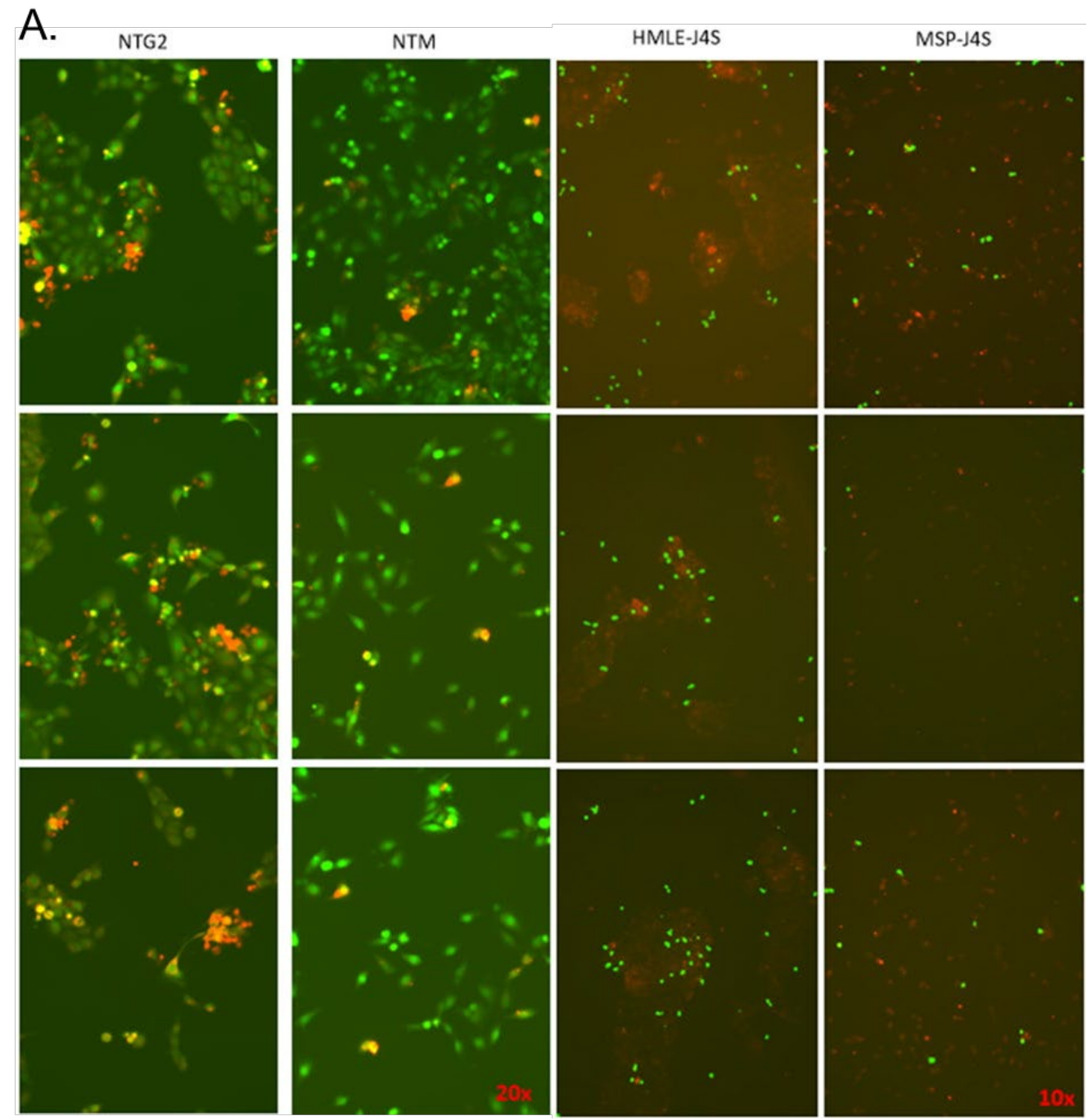

B.

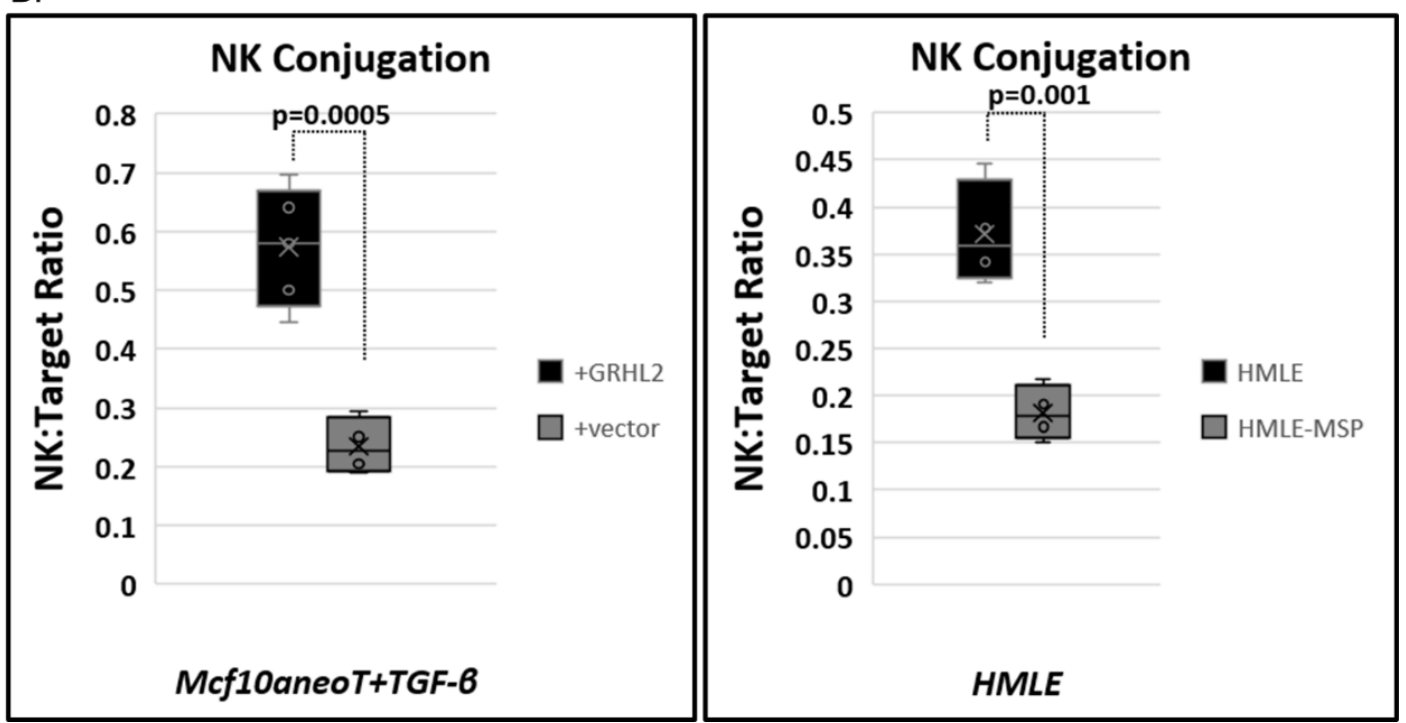


C.

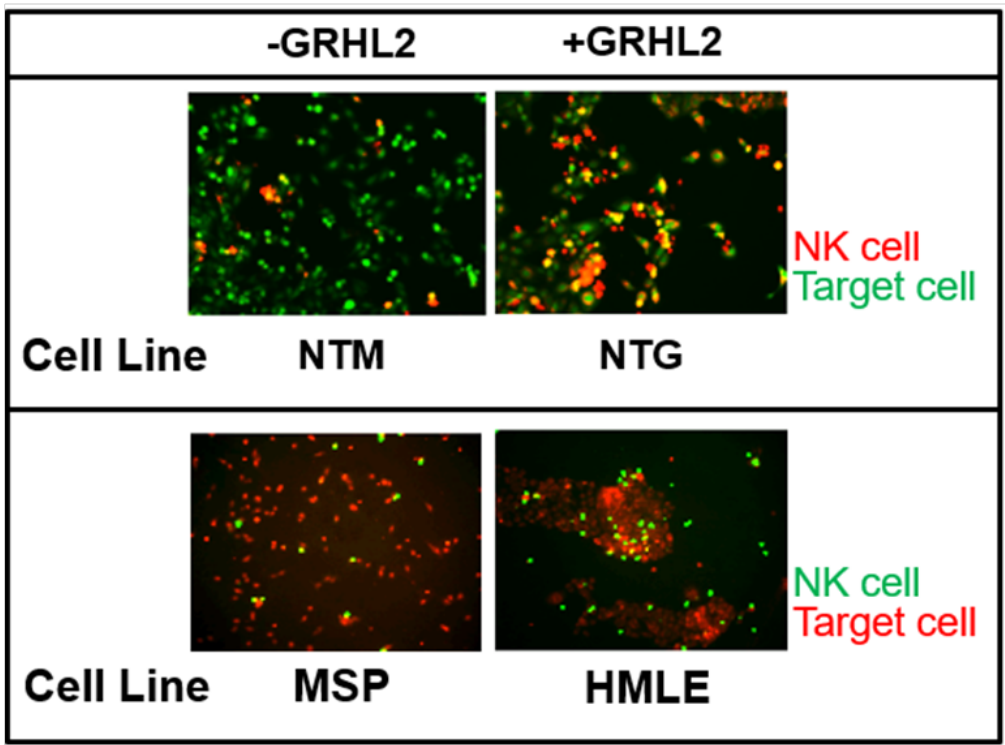

D.

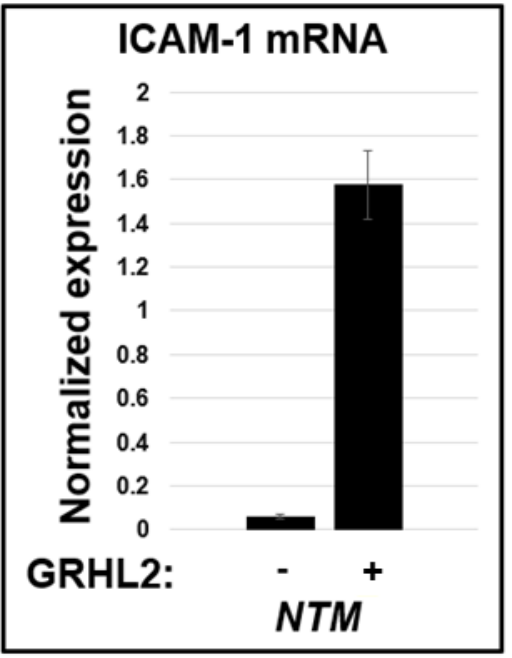

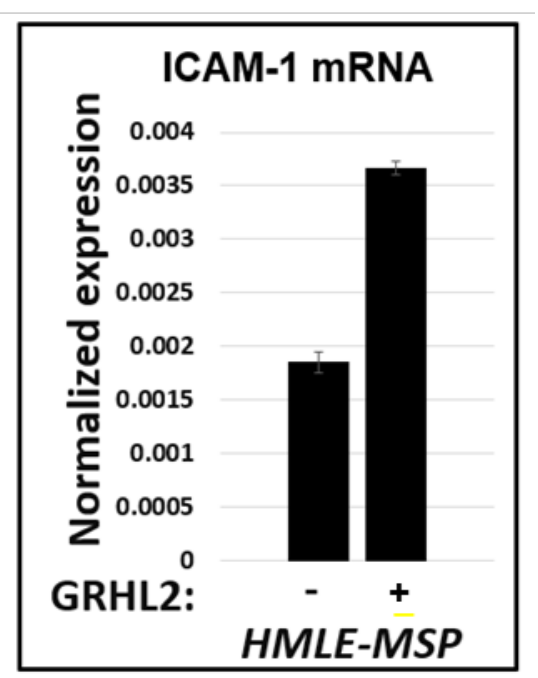



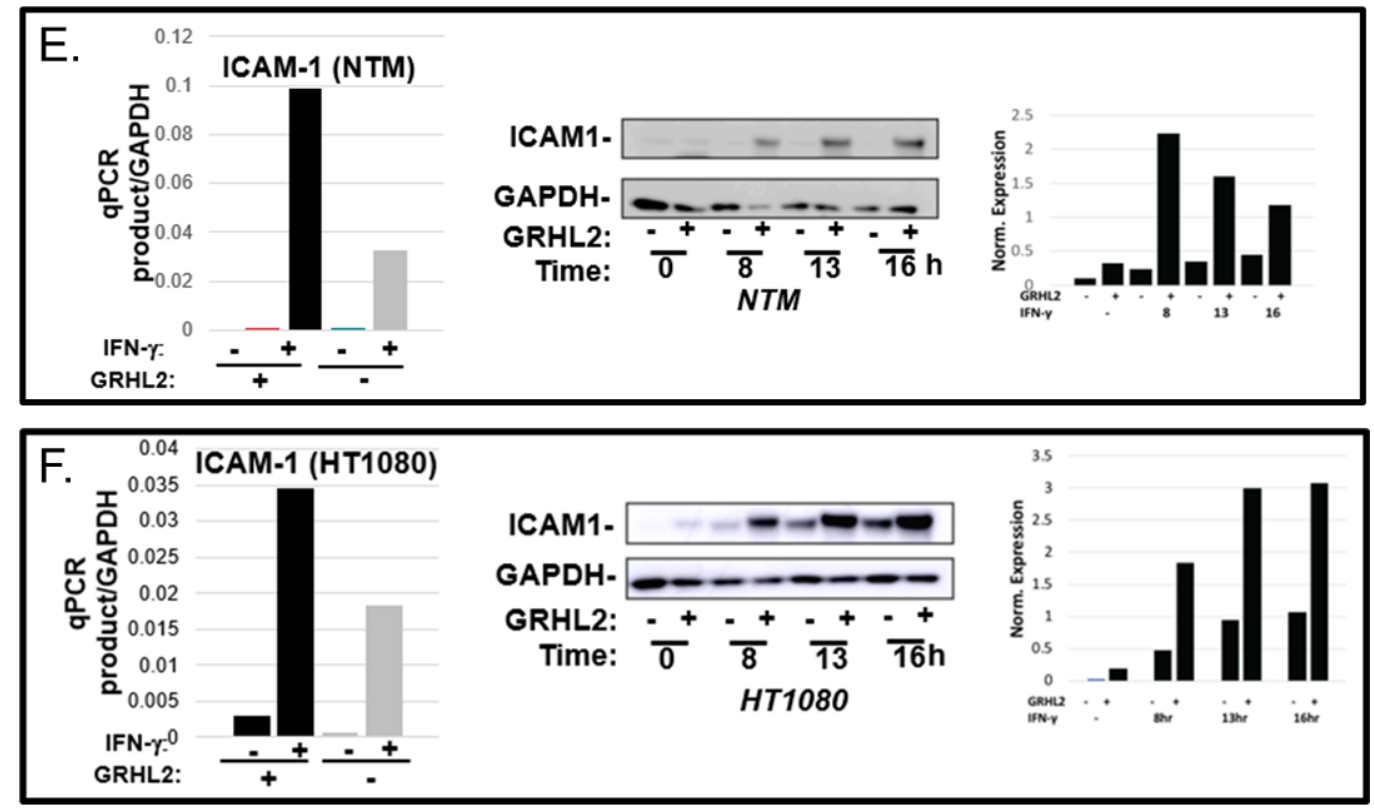

G.

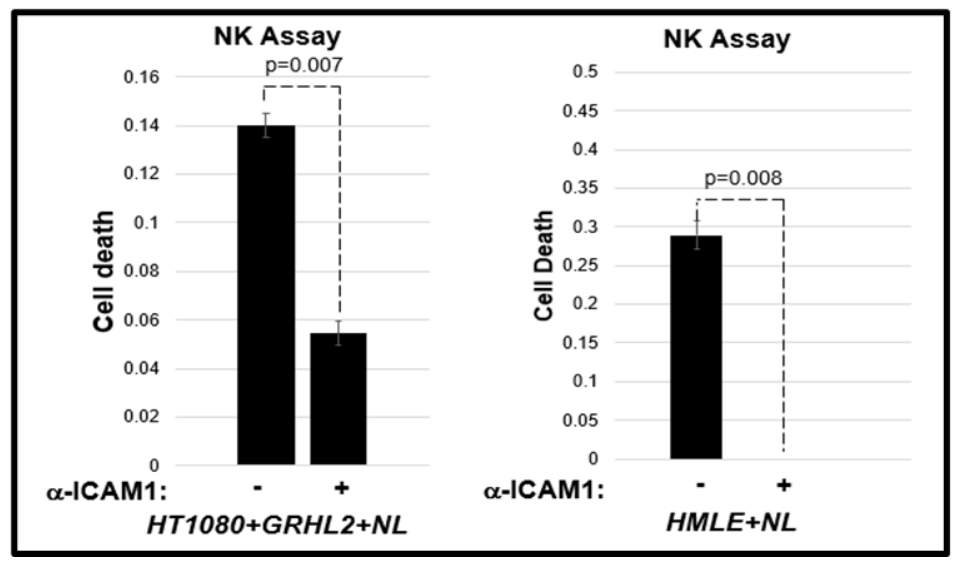

Figure 6. GRHL2 promotes NK-target conjugation via ICAM1 upregulation. A.) Mcf10aneoT+TGFB+vec ("NTM") or Mcf10aneoT+TGF-B+GRHL2 ("NTG2") and HMLE or HMLE-MSP cells were evaluated for NK92mi conjugation occurrence. Target and NK cells were separately, transiently stained with either CMFDA or CMPTX. After washing, NK assays were conducted. At timepoints, wells were gently washed using HBSS to remove unattached NK cells and floating target cells. Remaining cells were visualized and quantified by fluorescence microscopy to produce NK:target cell ratio for all wells. B.) Quantification of images. NK92mi cells were stained red in the HMLE experiment, while NK92mi cells were stained red in the Mcf10aneot+TGF-B experiment. 
Quantification: NTM= fields:4, target cells: 307; NTG2=fields:5, target cells:446; HMLEMSP=fields:4, target cells: 1,874; HMLE=fields:4, target cells:2,108. Quantification of conjugation was calculated using a Student's Two Tailed T test. C. Representative images from NK conjugation assays. D.) qRT-PCR quantification of ICAM-1 mRNA expression in NTM vs. NTG2, HT1080+mxs vs. HT1080+GRHL2, and HMLE-MSP vs. HMLE-MSP+GRHL2. E,F..) ICAM1 mRNA and protein before and after stimulation with IFN- $p$ was measured in HT1080, HT1080+GRHL2 and Mcf10aneoT+TGF-B, Mcf10aneoT+TGF-6+GRHL2 by qPCR normalized to GAPDH. For western blots, cell lysates were made before and after treatment with recombinant IFN- $y$ and western blotted, probed using indicated antibody, and quantified after normalization to GAPDH. D.) HT1080+GRHL2 and HMLE cells were pre-coated with ICAM1 blocking antibody, washed, then subjected to NK assays. Statistical significance calculated using Student's Two Tailed T test. Assay Details: G1: Duplicate. G2: Duplicate

\section{1.f. GRHL2 regulates NK sensitivity through epigenetic interactions.}

GRHL2 exerts influence over its target genes through interactions with epigenetic modifiers (Pifer et al., 2016). The lab had previously demonstrated that GRHL2 inhibits the histone acetyltransferase p300 as indicated by targeted loss of Histone 3 Lysine 27 acetylation (H3K27ac) at GRHL2-repressed genes, but not global H3K27ac loss (Pifer et al., 2016). Results from a Yeast 2 Hybrid screen previously conducted in the lab revealed that GRHL2 also likely interacted with the Histone 3 Lysine 4 mono-methylating enzymes KMT2C and KMT2D, whose interactions were previously thought to be most strongly implicated in nuclear receptor function (Gala et al., 2018). Abundance of H3K4me1 is a hallmark of active enhancer regions. Development of a GRHL2 non-interacting mutant termed "ED $\rightarrow A A$ " (indicating loss of 
interaction upon mutation of AA's 32 and 33 from glutamine and aspartic acid to alanine and alanine) had previously been generated and validated in the lab (MacFawn et al., 2019). We also used the previously generated and validated p300-non inhibiting GRHL2 mutant termed $\Delta 425-437$ (Pifer et al., 2016). We separately expressed both mutants in the mesenchymal cell lines HT1080, HMLE-MSP and Mcf10aneoT+TGF- $\beta$. After validating mRNA expression and equivalent protein expression by western blot, we characterized the ED $\rightarrow$ AA and $\Delta 425-437$ cell lines in 5 ways.

[1.] Morphological analysis: both ED $\rightarrow$ AA and $\Delta 425-437$ GRHL2 mutants showed reduced adoption of epithelial cell morphology compared with GRHL2 WT overexpression, which typically show a visible MET phenotype (Fig. 7, a). [2.] qRT-PCR of classic GRHL2regulated epithelial target genes indicated the inability of either ED $\rightarrow$ AA or $\Delta 425-437$ to induce mRNA expression above control in Mcf10aneoT+TGF- $\beta$ cells (RAB25, CDH1, ESRP1, OVOL2, TP63, CLDN4). (Fig. 7, b) [3.] Both mutants showed altered sensitivity to anoikis compared with GRHL2 WT (Fig. 7,c) The ED $\rightarrow$ AA mutant seemed to show an intermediate phenotype between GRHL2 WT and GRHL2 (-) [4.] Analysis of genes with overlapping GRHL2/KMT2C binding sites (according to UCSC Genome Browser) by CHIP-qPCR using a CHIP validated KMT2C antibody revealed that $\mathrm{ED} \rightarrow \mathrm{AA}$ GRHL2 cells were not able to recruit $\mathrm{KMT} 2 \mathrm{C}$ to these select promoters without a GRHL2 interaction in Mcf10aneoT+TGF- $\beta$ cells. This shows that GRHL2 is essential for KMT2C recruitment at particular genes that have binding sites for both GRHL2 and KMT2C (TP63, CLDN4, RAB25, CDH1, OVOL2). (Fig 7, d) [5.] Functional assays to determine characteristics of the ED $\rightarrow$ AA and $\triangle 425-437$ GRHL2 mutants revealed that both mutants were resistant to anoikis (Mcf10aneoT+TGF-b) and NK attack (Mcf10aneoT+TGF-b, HMLE-MSP) in a 
manner similar to empty vector control cells, in contrast to strong sensitization observed with GRHL2 WT overexpression. (Fig. 7 e,f).

We also explored the effects of KMT2C/D knockout using an Mcf10a series that had KMT2C/D homozygous or heterozygous knockouts, previously generated by others in the lab. Messenger RNA expression levels of classical epithelial and mesenchymal genes were mostly unchanged by homozygous knockout of $\mathrm{KMT2C}$, however, when this was combined with a heterozygous knockout of KMT2D, we noticed an inversion of gene expression signature, with epithelial genes no longer expressed (CDH1, GRHL2, OVOL, ESRP, RAB25 ICAM-1), and mesenchymal genes concurrently turned on (ZEB1, Collagen 8a, SRGN, DCN, MMP2) (Fig. 7, g,h). KMT2C-/-/KMT2D+/- cells also exhibited a profoundly altered morphology, with an elongated, spindly appearance, further evoking an EMT phenotype (Fig. 7, i). This suggests that the histone methyltransferases KMT2C and KMT2D are utilized by lineage defining machinery, perhaps most notably GRHL2, whose downregulation upon KMT2C/D knockout suggests a selfreinforcing positive feedback loop. We were curious to see if this EMT induced by $\mathrm{KMT}^{-\mathrm{C}^{-/}-}$ /KMT2 $\mathrm{D}^{+/-}$would also confer resistance to NK attack. Curiously, we found that the $\mathrm{KMT}^{2} \mathrm{C}^{-/-}$ $/ \mathrm{KMT}_{2} \mathrm{D}^{+/+}$cells were sensitized, while the $\mathrm{KMT} 2 \mathrm{C}^{-/-} / \mathrm{KMT}_{2} \mathrm{D}^{+/-}$cells were comparatively much more resistant, but at a level similar to control (Fig. 7, j). This experiment warrants further investigation. 


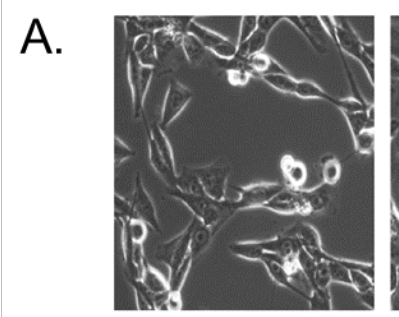

GRHL2:

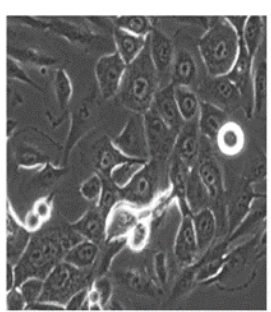

WT

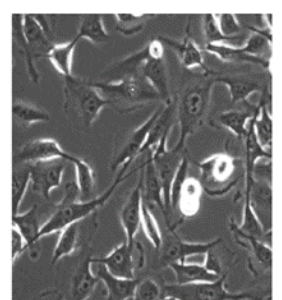

$\triangle 425-437$

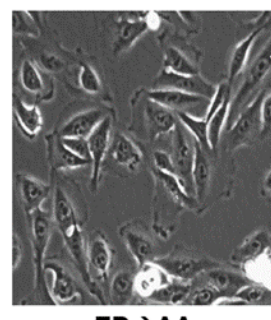

ED $\rightarrow$ AA
B.

Mcf10aneoT+TGF- $\beta$
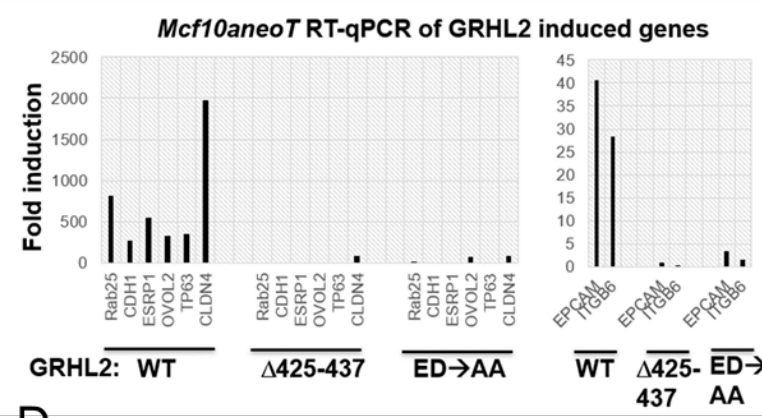

D.

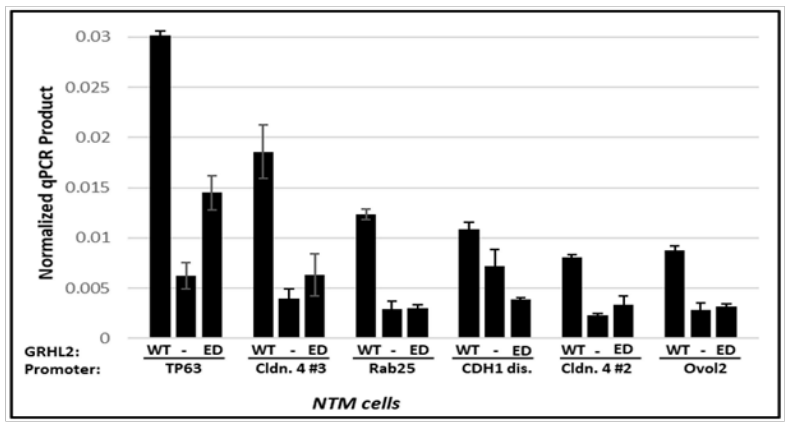

F.
C. 16hr Anoikis Assay
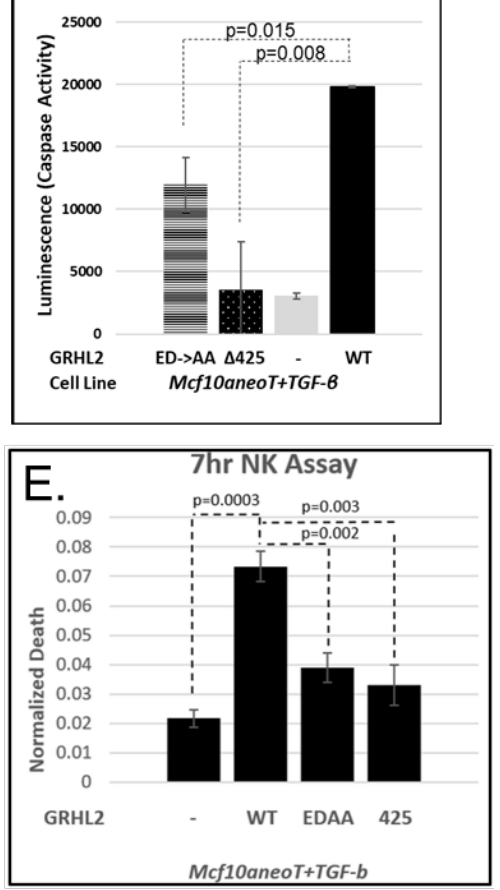

Mcf10aneoT+TGF-b

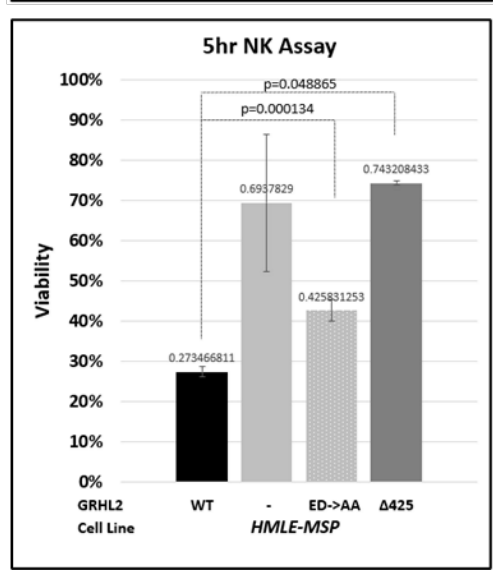


G.
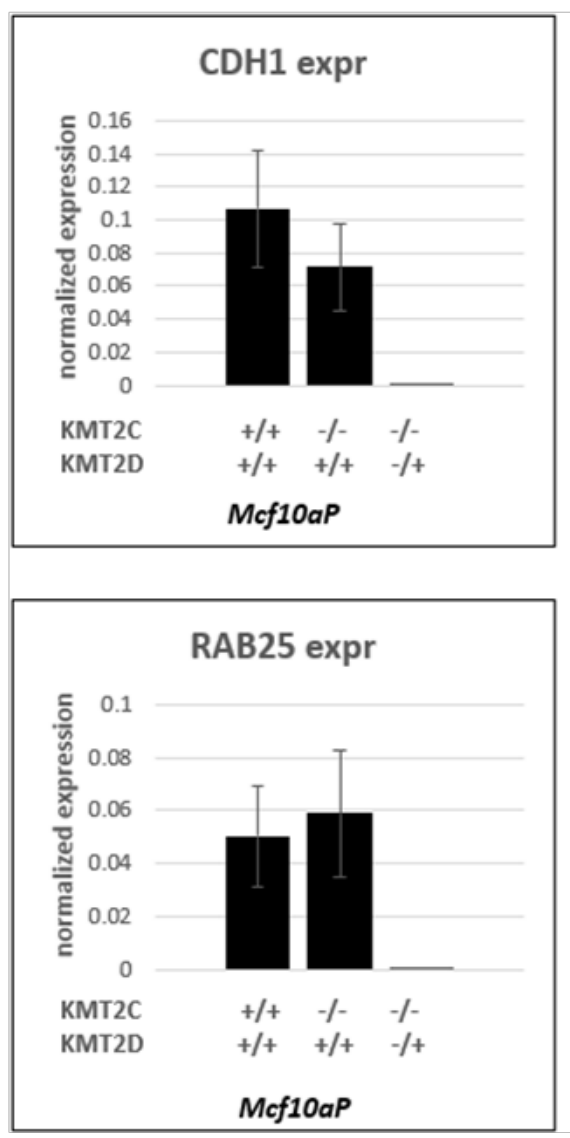

Epithelial Genes (mRNA Expression) expression)
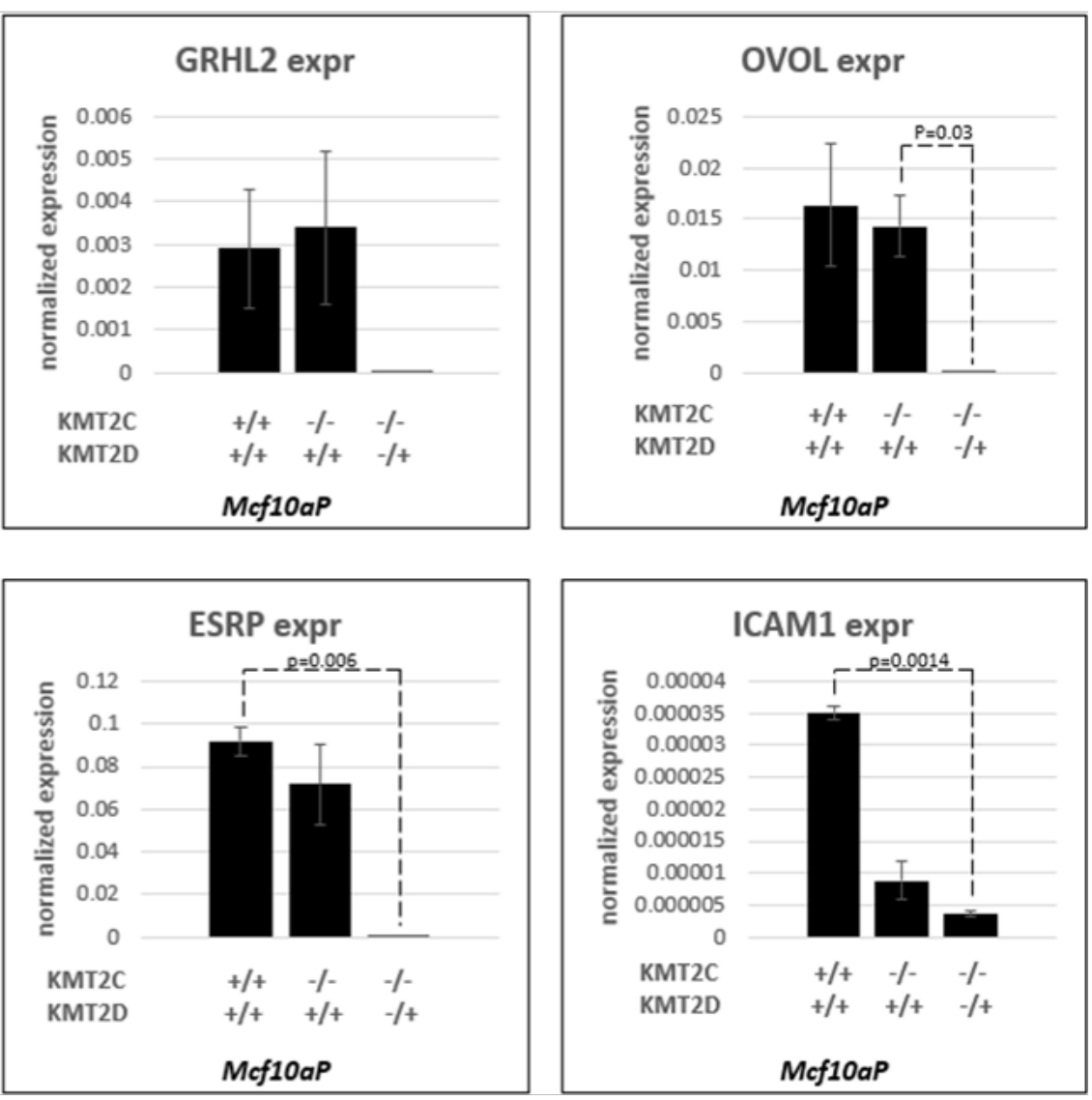

$\mathrm{H}$.
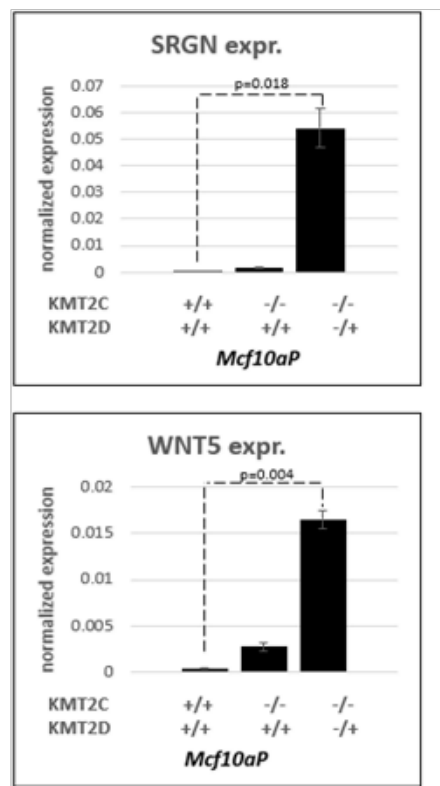

\section{Mesenchymal Genes (mRNA Expression) expression)}
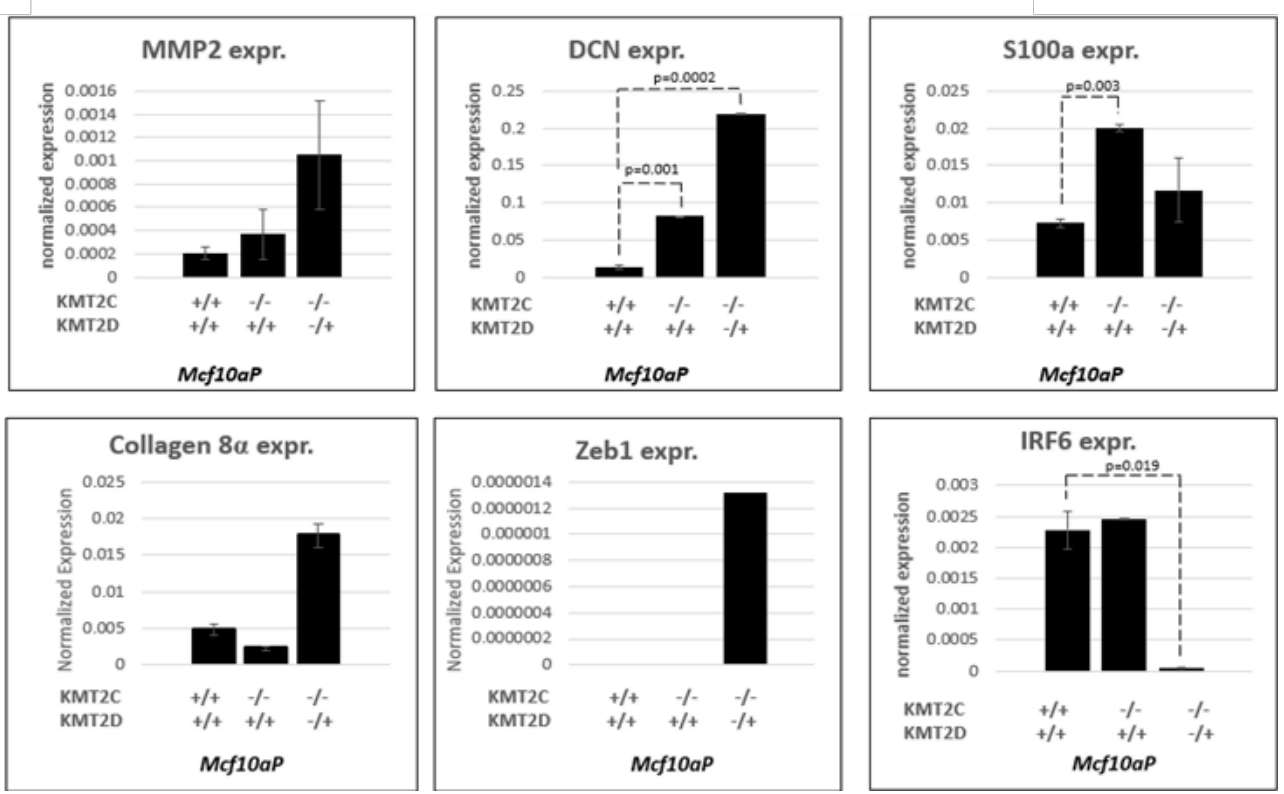
I.

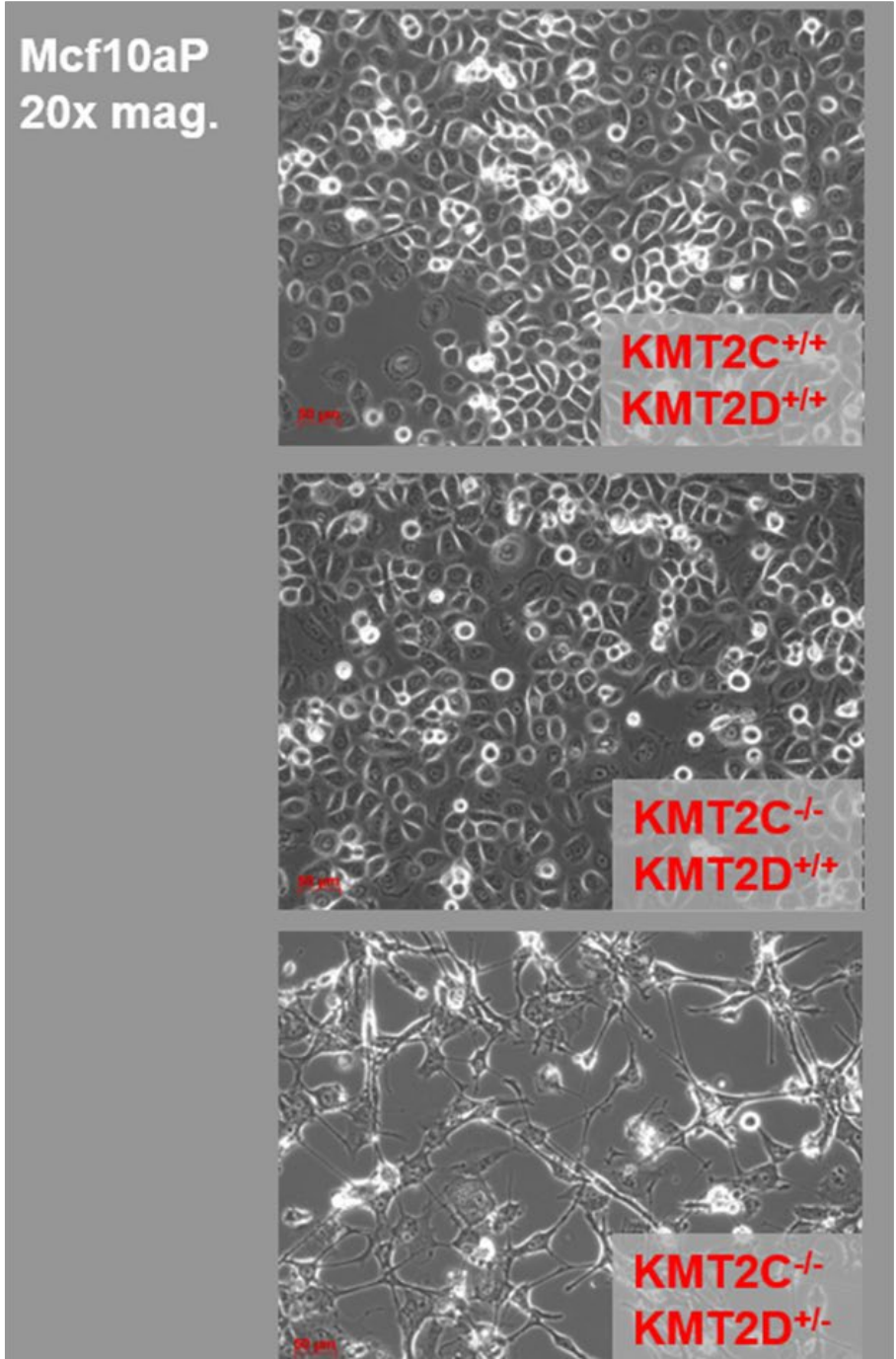

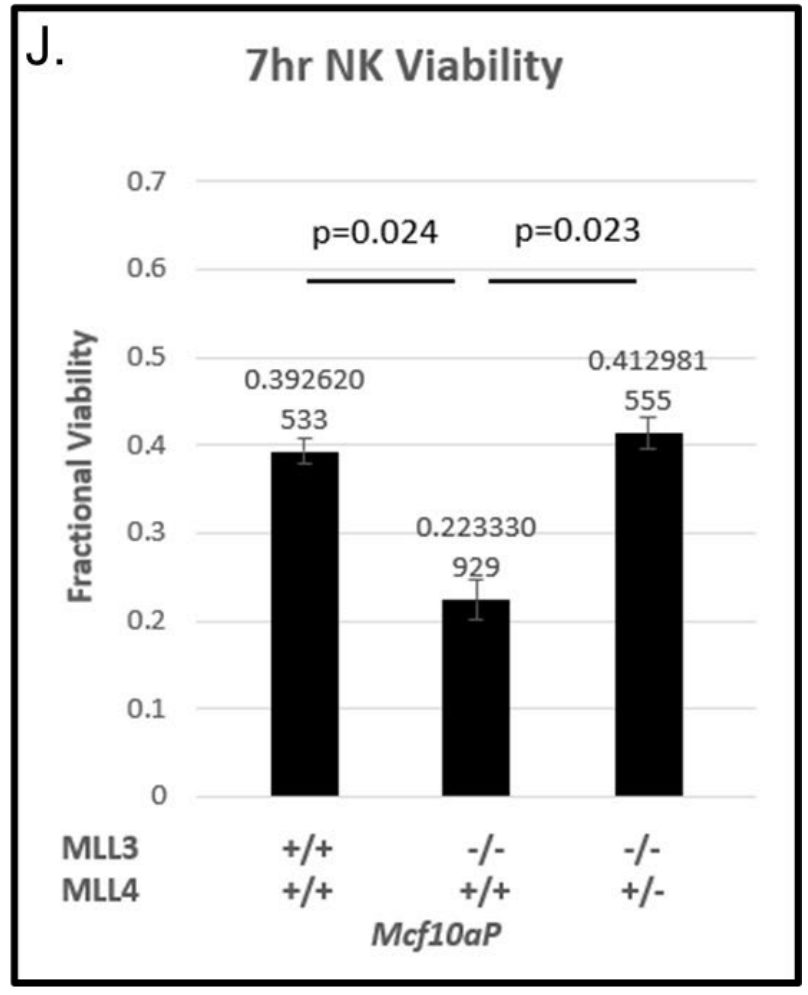

Figure 7. KMT2C/D interaction is important for GRHL2-mediated NK sensitization. A.)

Morphological brightfield images of Mcf10aneoT+TGF-6 (+GRHL2 WT/ EDAA / 4425$)$ with

ectopically expressed GRHL2 mutants plated at subconfluent densities. B.) qPCR measure of mRNA transcripts of various GRHL2-induced genes in cells containing GRHL2 WT versus mutants. C.) Anoikis assay conducted on Mcf10aneoT+TGF-6 stably expressing GRHL2 WT or mutants were suspended in Mcf10a medium $+10 \%$ methylcellulose, then assayed using Caspase 3/7 glo signal at timepoints. D.) CHIP-qPCR measure of KMT2C occupancy of select promoters containing binding sites for both GRHL2 and KMT2C in cells with WT, unexpressed, or EDAA 
mutants indicates loss of KMT2C recruitment in the absence of GRHL2 or GRHL2 interaction. D.) Anoikis assay on Mcf10aneoT+TGF-B+vec ("NTM") with GRHL2 WT or mutants ectopically expressed. Assay conducted with a minimum of 2 technical duplicates per cell line. E,F.) NK assay on Mcf10aneoT+TGF- 6 or HMLE-MSP with ectopically expressed GRHL2 WT or mutants. NK assays were conducted in triplicate, statistical analysis was conducted using a Student's Two-tailed T test. G,H.) qPCR mRNA expression in Mcf10aP KMT2C/D Crispr-Cas9 K.O. cells reveals that epithelial/mesenchymal gene expression patterns are de-stabilized when KMT2C and KMT2D are altered. I.) Brightfield phase-contrast microscopy at 20X magnification. J.) NK assay conducted on KMT2C (MLL3) and KMT2D (MLL4) versus WT Mcf10aP cells. Cells were plated at $4.5 \times 10^{4}$ cell per well in duplicate. NK92mi cells were added at a 3:1 ratio and assayed for Thrs. Student's Two-tailed T test was used for statistical analysis.

Taken together, this data reveals that GRHL2's direct interaction with KMT2C/D is required for activation of epithelial gene expression and adoption of functional characteristics typical of the epithelial state (epithelial morphology, anoikis sensitivity, NK sensitivity). We proposed that GRHL2 is an important mediator of immune sensitivity, and that this vulnerability is mediated, at least in part, through maintenance of ICAM-1 expression. QPCR and western blot analysis indicated that neither ED $\rightarrow$ AA nor $\triangle 425-437$ induced ICAM-1 expression to the levels that WT GRHL2 does in Mcf10aneoT+TGF- $\beta$ cells (Fig. 8, a,b). KMT2C-1/KMT2 $\mathrm{D}^{+/-}$mutant cells, in the presence of WT GRHL2, also exhibited reduced ICAM-1 mRNA expression (Fig 7. g). Further, UCSC Genome Browser KMT2C and GRHL2 tracks suggested overlapping binding in the ICAM-1 promoter. Similar to other promoters containing GRHL2/KMT2C overlap, KMT2C 
occupancy on this promoter was reduced in ED $\rightarrow$ AA GRHL2 mutant cells as assayed by CHIPqPCR using a KMT2C CHIP-validated antibody (Fig 8. d,e).

To conclude, utilization of a GRHL2 KMT2C/D non-interacting mutant previously generated in our lab, emphasizes the importance of KMT2C/D in GRHL2's regulation of gene expression. Not only does GRHL2 depend on KMT2C/D to activate epithelial gene expression, but KMT2C is dependent upon GRHL2 in order to be recruited to a subset of genes that contain GRHL2 and KMT2C binding sites, as demonstrated by loss of KMT2C binding in CHIP-qPCR experiments. These results also determine that NK-sensitizing gene expression in some epithelial lineages is under positive regulation from GRHL2/KMT2C/D because GRHL2 ED $\rightarrow$ AA mutant, which induced an EMT gene expression program, exhibited altered resistance to NK attack. 

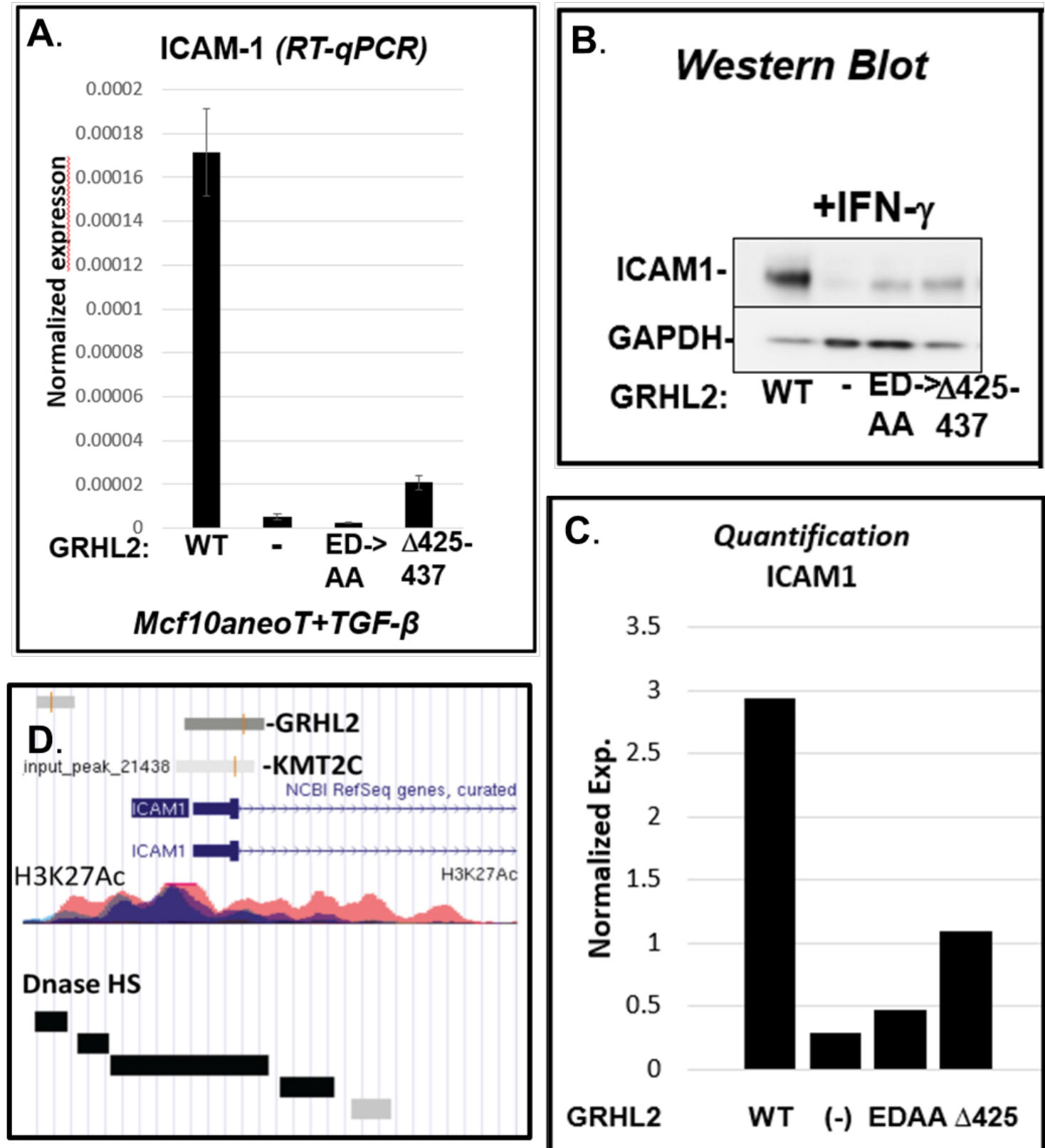

\section{Quantification ICAM1}

3.5
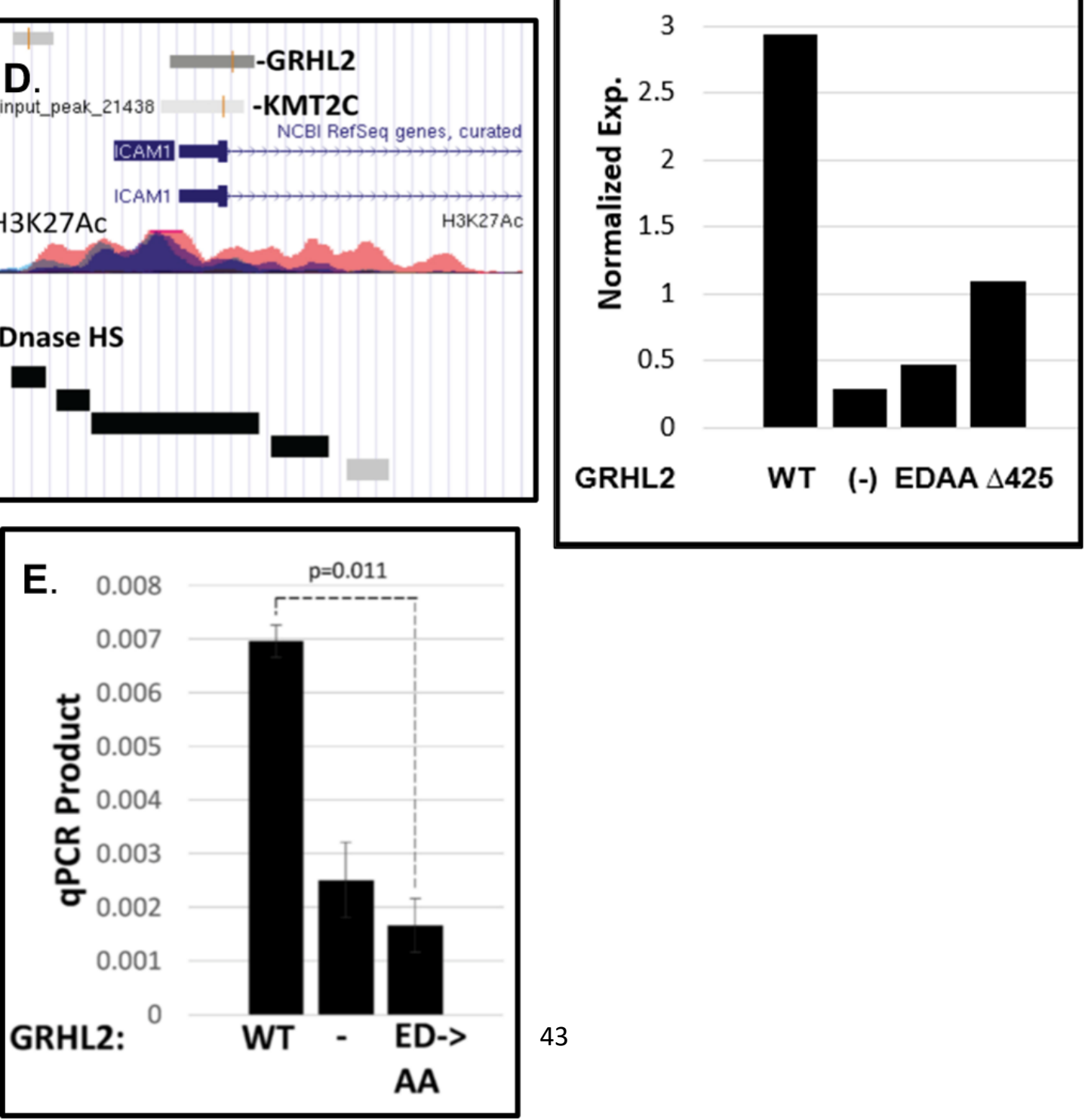
Figure 8. GRHL2 and KMT2C interaction is necessary for ICAM1 mRNA and protein expression. A.) qPCR expression of ICAM1 mRNA indicates inability of GRHL2 mutants to activate gene expression significantly above control when stably expressed in Mcf10aneoT+TGF-6 cells. B.) Western blot on Mcf10aneoT+TGF-B cells stimulated with IFN- $\gamma$ demonstrates reduced capacity for ectopic GRHL2 mutants to activate ICAM1 protein expression. C.) Quantification normalized to GAPDH signal. D.) UCSC Genome Browser tracks indicate overlap of GRHL2 and KMT2C predicted binding sites from curated CHIP-seq data near promoter region of ICAM1. E.) qPCR product from CHIP-qPCR in Mcf10aneoT+TGF-6 using Chip-validated KMT2C antibody demonstrates lack of KMT2C localization to ICAM1 genomic region in cells stably expressing GRHL2 non-interacting mutant (EDAA).

\section{I.g. EMT signature anti-correlates with Interferon signature in Lung Adenocarcinoma patient samples (TCGA).}

The preliminary data involving ICAM1 and interferon response prompted us to check The Cancer Genome Atlas (TCGA) for correlations between epithelial state and interferon response in the context of clinical patient samples. We selected Lung adenocarcinoma patients because it is known to be an immune-infiltrated cancer type. Patient samples were stratified into quartiles based upon their expression of an 18-gene EMT signature (Gibbons \& Creighton, 2018). EMT score was then correlated with a panel of 47 Interferon-related genes, after their expression was normalized to IFN- $\gamma$ transcript levels within each patient sample. This normalization should correct for differences in immune infiltrate and cytokine stimulation. GRHL2 was used as a positive control for a low EMT score. Correlation was calculated as deviation from a random distribution (0.5:0.5) after normalization. Intriguingly, our analysis showed that low EMTscore 
(Q1) correlated with high IFN-pathway gene

expression (Q4) for the majority of the genes analyzed

(Table 2). Patients with tumors that have a high EMT

score (Q1) correlated with lower IFN-pathway gene

expression. Although correlative, this data supports

the notion that the epithelial state is better suited to

amplify interferon response. This could be a result of

tumor microenvironmental modulation, such as

chemokine production and promotion of immune cell

recruitment, and/or could reflect tumor cell intrinsic

maintenance of interferon response upon stimulation

by immune cell-secreted cytokines.

Table 2: EMT anti-correlates with IFN-related genes in lung cancer. After quartile grouping according to EMT

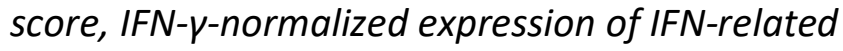
genes was found to anti-correlate with EMT score.

Random distribution $=(0.5: 0.5)$.
EMT Q1 vs. EMT Q1 vs.

IFN gene: Q1 express. Q4 express. adj. p-value GRHL2 CAMK2G

OAS3

PIAS1

PRKCD

IFNLR1

MAPK3

IFIH1

HERC6

OAS1

MAP2K1

MX1

IRF3

CBL

DDX58

RNASEL

IFIT1

RAP1B

AKT1

IFI6

OAS2

IRF7

IFIT2

IFI27

IRF1

SAMD9

IFIT3

CEBPB

PIK3R1

FASLG

IFI44L

ISG15

OASL

TRIM22

IFI44

CASP1

ICAM1

IRF5

RSAD2

CDKN1A

SOCS1

HERCS

CAMK2A

LAG3

CD274

CAMK2B

CxCl10

CXCl9

\begin{tabular}{|c|c|c|}
\hline 0.18 & 0.82 & $2.3 E-06$ \\
\hline 0.20 & 0.80 & $6.4 E-06$ \\
\hline 0.20 & 0.80 & $3.4 E-05$ \\
\hline 0.23 & 0.77 & $8.5 E-05$ \\
\hline 0.24 & 0.76 & $8.5 E-05$ \\
\hline 0.23 & 0.77 & 9.1E-05 \\
\hline 0.24 & 0.76 & $1.3 E-04$ \\
\hline 0.25 & 0.75 & $1.5 E-04$ \\
\hline 0.26 & 0.74 & 2.1E-04 \\
\hline 0.24 & 0.76 & 2.1E-04 \\
\hline 0.26 & 0.74 & 2.1E-04 \\
\hline 0.24 & 0.76 & 2.1E-04 \\
\hline 0.25 & 0.75 & 2.1E-04 \\
\hline 0.26 & 0.74 & $2.9 E-04$ \\
\hline 0.25 & 0.75 & $2.9 E-04$ \\
\hline 0.25 & 0.75 & $2.9 E-04$ \\
\hline 0.26 & 0.74 & $4.2 \mathrm{E}-04$ \\
\hline 0.28 & 0.72 & $5.6 \mathrm{E}-04$ \\
\hline 0.28 & 0.72 & $6.6 E-04$ \\
\hline 0.27 & 0.73 & 6.7E-04 \\
\hline 0.27 & 0.73 & 6.7E-04 \\
\hline 0.29 & 0.71 & $7.1 E-04$ \\
\hline 0.28 & 0.72 & $7.6 \mathrm{E}-04$ \\
\hline 0.29 & 0.71 & $8.8 \mathrm{E}-04$ \\
\hline 0.29 & 0.71 & $8.8 \mathrm{E}-04$ \\
\hline 0.28 & 0.72 & $1.2 \mathrm{E}-03$ \\
\hline 0.29 & 0.71 & $1.4 E-03$ \\
\hline 0.31 & 0.69 & 2.7E-03 \\
\hline 0.31 & 0.69 & $3.0 \mathrm{E}-03$ \\
\hline 0.30 & 0.70 & 3.7E-03 \\
\hline 0.32 & 0.68 & 4.1E-03 \\
\hline 0.32 & 0.68 & 4.7E-03 \\
\hline 0.32 & 0.68 & 7.7E-03 \\
\hline 0.33 & 0.67 & $8.0 E-03$ \\
\hline 0.34 & 0.66 & $8.8 \mathrm{E}-03$ \\
\hline 0.34 & 0.66 & $1.1 \mathrm{E}-02$ \\
\hline 0.35 & 0.65 & $1.9 \mathrm{E}-02$ \\
\hline 0.36 & 0.64 & $2.0 E-02$ \\
\hline 0.36 & 0.64 & $2.2 E-02$ \\
\hline 0.36 & 0.64 & $2.2 \mathrm{E}-02$ \\
\hline 0.38 & 0.63 & $2.6 \mathrm{E}-02$ \\
\hline 0.37 & 0.63 & $2.9 E-02$ \\
\hline 0.38 & 0.62 & $4.2 \mathrm{E}-02$ \\
\hline 0.44 & 0.56 & $2.1 E-01$ \\
\hline 0.45 & 0.55 & $2.5 E-01$ \\
\hline 0.45 & 0.55 & 2.7E-01 \\
\hline 0.55 & 0.45 & $3.1 E-01$ \\
\hline 0.52 & 0.48 & $4.5 E-01$ \\
\hline
\end{tabular}

0 


\section{1.h. GRHL2 protein expression correlates with time to relapse in Breast cancer}

Analysis of patient data across cancer types reveals a context-dependent role for GRHL2 in predicting clinical outcomes. "KMplot.com" houses a free, online Kaplan-Meyer plotting software that can be used to query mRNA expression across multiple databases (TCGA, GEO, etc.), correlating expression with clinical characteristics (Nagy, Lanczky, Menyhart, \& Gyorffy, 2018). We utilized this database to produce KM plots for GRHL2 using the "pan-Cancer" setting. Lung SCC (HR: 0.7), stomach adenocarcinoma (HR:0.6) and bladder carcinoma (HR: 0.7) exhibit statistically significant better overall survival in patients with GRHL2 mRNA above median. Conversely, Kidney (HR:1.9), Breast cancer (HR:1.7), and Pancreatic ductal adenocarcinoma (HR: 2.4) correlate high GRHL2 mRNA with worse overall survival (Fig.9 a,b). The dichotomous role of GRHL2 clearly requires in vivo experiments to better understand the context-dependent nature of itsfunction.

The Frisch lab had previously devised and initiated a transgenic GRHL2 murine recurrent mammary tumor model. The model was developed by Josh Farris MD,PhD, Philip Pifer MD, PhD and Steven Frisch PhD. Later stages of the project weremaintained by Hannah Wilson PhD and the author. Final data analysis was conducted by Steven Frisch PhD and the author (unpublished data: Pifer P., Farris J., Wilson H., Frisch S.M.). Mice expressing a mammary gland-specific MMTV-GRHL2 transgene were crossed with tetO-neuNT;MMTV-tTA (MTB/TAN) mice. Upon administration of doxycycline, mice develop neu oncogene-driven mammary tumors. GRHL2(+) and control mice exhibit analogous tumor formation. Removal of doxycycline causes efficient regression of primary tumors in this model. Mammary glands with MMTV driven GRHL2 expression 
showed almost a complete lack of recurrent tumors (0-7\%), compared with control mice (29.4\%). Unfortunately, this experiment did not reach statistical significance which likely stemmed from two problems; 1.) The $p$-value if comparing MMTV-GRHL2 (0/13) versus control (5/17) recurrence was 0.052 . A larger number of mice would be advisable for repeating this experiment. 2.) One of the mice in the MMTV-GRHL2 tumor did have a recurrence, although the recurrence, unexpectedly, did not express GRHL2, although it was anatomically located in the mammary gland. Including this mouse in the recurrence data would mean that $1 / 13(7 \%)$ of MMTV-GRHL2 mice had recurrence compared to $5 / 17(29.4 \%)$ in control (Fig 9.d). This is not a statistically significant result. Because the experiment did not reach significance in a reliable way, we cannot publish the results and the data must be interpreted with caution, however the results show a trend towards GRHL2 suppressing recurrence and warrants further investigation of this phenomenon. Future studies should use a higher (n) of mice because although the number of mice developing a primary tumor was high, the recurrence rates in both groups was low, making statistical analysis challenging within the sample sizes. 

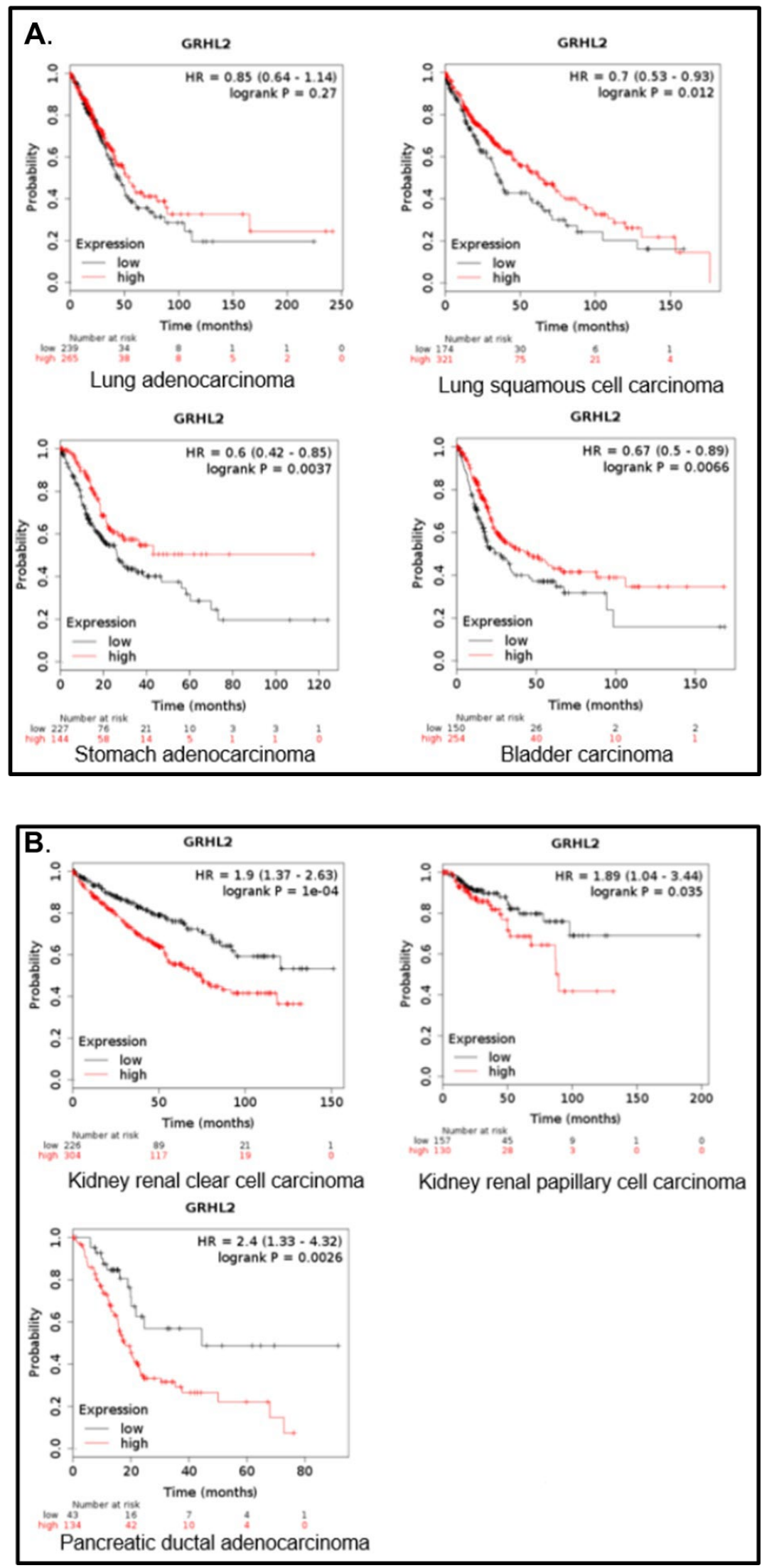


\section{C. \\ Mouse Tumor Recurrence}

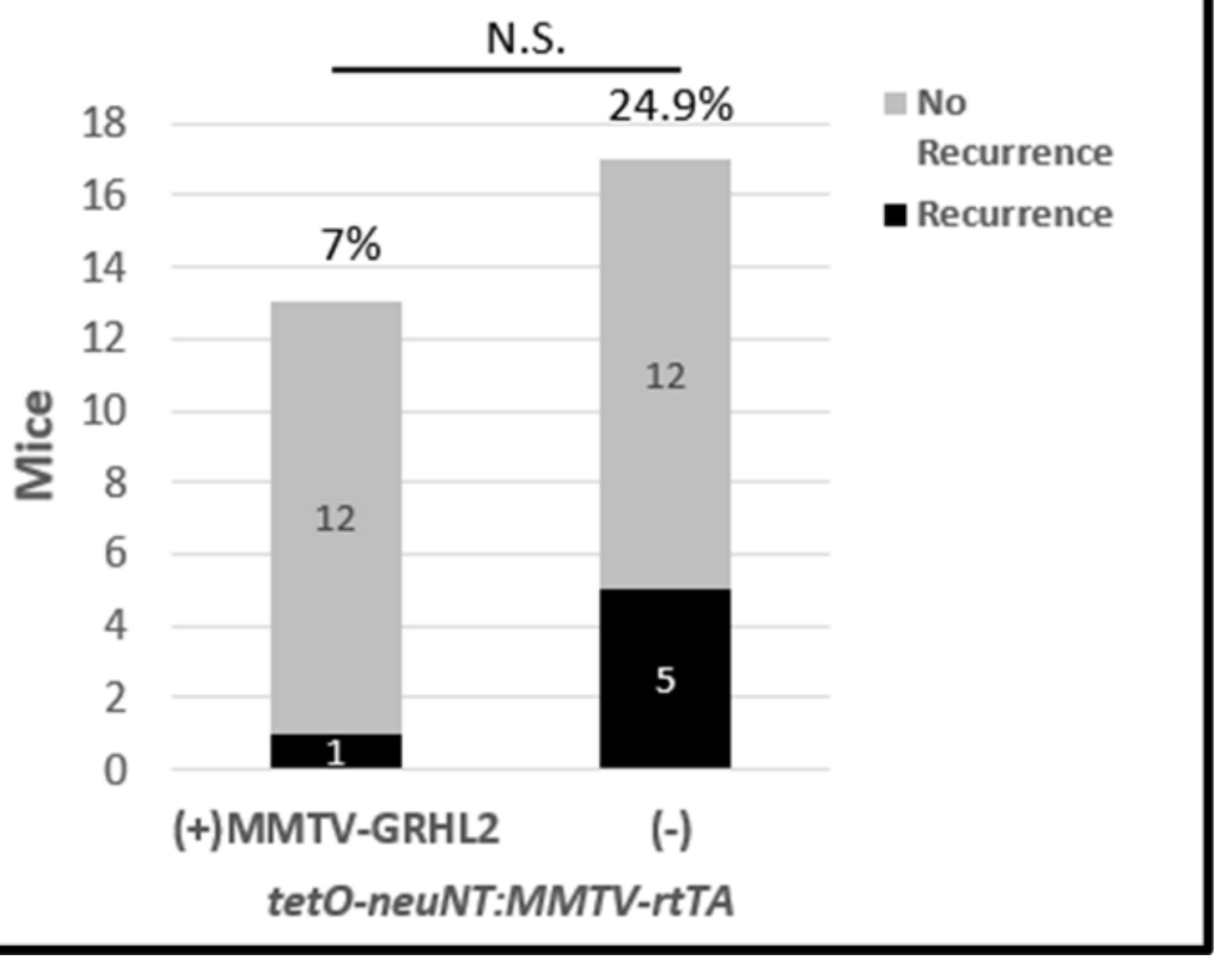

Figure 9. GRHL2 mRNA correlations in patient databases. Using the "pan-cancer" RNAseq option, we plotted Overall Survival against GRHL2 mRNA expression for various cancer types. A.) GRHL2 mRNA correlates with better OS in Lung Squamous Cell Carcinoma $(H R=0.7 p=0.012)$, Stomach adenocarcinoma $(H R=0.6, p=0.0037)$, and bladder carcinoma (HR=0.67, $p=0.0067)$. B.) GRHL2 mRNA correlates with worse OS in Kidney renal clear cell carcinoma (HR=1.9, $p=0.0004)$, Kidney renal papillary cell carcinoma $(H R=1.89, p=0.035)$, and Pancreatic ductal adenocarcinoma $(H R=2.4$, p=0.0026). C.). A mouse MMTV-GRHL2 recurrent tumor model produced a trend towards 
lower rates of recurrence in mice expressing mammary-gland specific GRHL2, although the results did not reach statistical significance ( GRHL2- 7\% recurrence rate / control$24.9 \%$ recurrence rate). The mouse tumor model was initiated by Philip Pifer MD, PhD, Josh Farris MD, PhD, and Steven Frisch PhD, latter stages of the project were maintained by Hannah Wilson, PhD. 


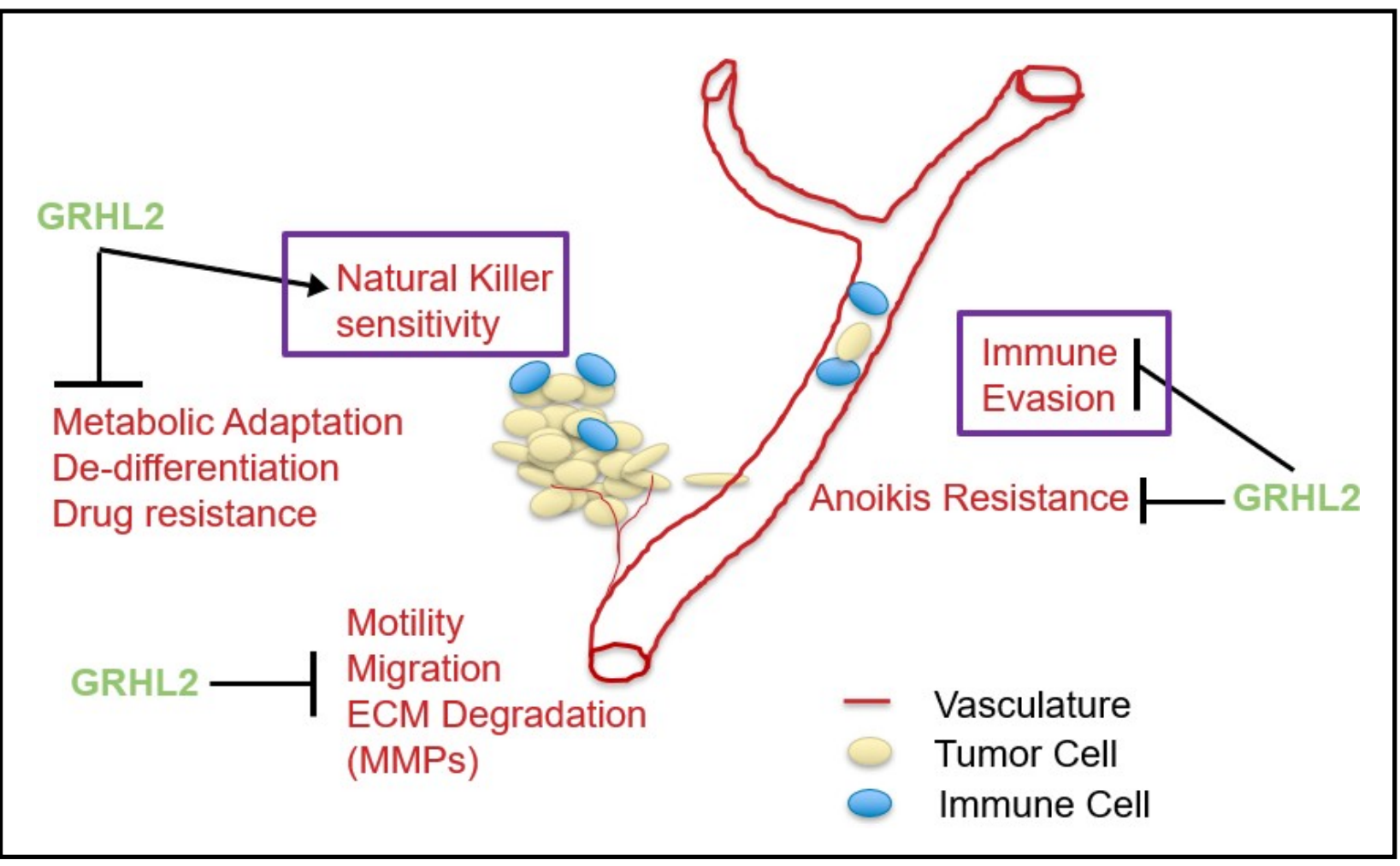

Diagram 3: The significance of GRHL2 in tumor biology. Novel proposed roles of GRHL2 boxed in purple.

Our data supports the hypothesis that GRHL2's epithelial programming includes maintenance of pathways promoting sensitivity to NK-mediated cell death. The epithelial, rather than mesenchymal state, in Mcf10aneoT, HMLE, HT1080, and BT549 cells exhibited enhanced sensitivity to cell-mediated cytotoxicity by human Natural Killer cells. Numerous avenues potentially directing sensitivity were explored, but ICAM-1 expression appeared to be the most consequential in cells where the epithelial state was maintained by GRHL2 expression. ICAM-1 expression was epigenetically regulated by GRHL2's recruitment of KMT2C to the promoter of this gene, likely orchestrating permissive chromatin architecture for transcription. 
We showed that GRHL2 repressed autophagy in HT1080 fibrosarcoma cells, and repressed the chondroitin sulfate proteoglycan Serglycin in HT1080 and Mcf10aneoT+TGF- $\beta$ cells; though not significantly altering NK sensitivity in our model, these attributes may be consequential for other realms of tumor biology. We explored the role of epithelial versus mesenchymal matrix composition and stiffness, and hypothesize that differences may modulate target cell tenseness, influencing killing dynamics by NK cells.

Our data would predict GRHL2 dependent loss of ICAM-1 expression to be an avenue of immune evasion during the oncogenic EMT. Evidence from multiple models clearly demonstrate that hybrid or partial EMT can contribute to other tumorigenic properties such as plasticity, sphere-formation, tumor establishment, drug resistance, etc. (M. K. Jolly et al., 2019; Mohit Kumar Jolly et al., 2016; Strauss et al., 2011). It would be interesting to measure the levels of GRHL2 expression during these hybrid states, and consequently, ICAM-1 expression. Our data agrees with the well-documented central role of ICAM-1 in immune effector cellmediated cytolysis. Future studies should attempt to replicate these findings in other models of EMT/MET, especially in the context of circulating or disseminated tumor cells. Therapies aimed at preserving ICAM-1 expression could prove useful in the context of immunotherapies in order to ensure that checkpoint blockaded immune cells are still able to form immune synapses with their target cells. As mentioned above, irradiation has been shown to upregulate ICAM-1 expression, and a study conducted in acute myeloid leukemia (AML) showed reinvigorated NK cells caused paracrine activation of ICAM-1 on target cells (Parameswaran et al., 2016) (Jeong et al., 2018). This underscores the notion that ICAM-1 is responsive to, and reliant on, various stimuli, in addition to IFN- $\gamma$. Conversely, EMT has been associated with upregulation of the 
immune checkpoint molecule PD-L1 in various contexts including p53-mutant pancreatic cancer, cisplatin-resistant lung cancer, and gastric carcinoma (Shen et al., 2019; Song et al., 2020; Xu et al., 2019). Future experiments should query if GRHL2 expression, through ICAM-1 promotion, can boost response to PD-L1 checkpoint blockade in these contexts.

We demonstrated that GRHL2 enforces sensitivity to NK attack through its interactions with epigenetic modifiers, which it uses to promote expression of an NK-sensitizing pathway. To be clear, GRHL2 is not a frequently mutated gene according to patient databases (TCGA), however it's functional partners KMT2C and KMT2D are two of the most highly mutated genes across all cancers: $\mathrm{KMT} 2 \mathrm{D}=3^{\text {rd }}$ and $\mathrm{KMT} 2 \mathrm{C}=7^{\text {th }}$ according to the National Cancer Institute Genomic Data Commons (GDC) (Fagan \& Dingwall, 2019). KMT2C and KMT2D are generally regarded as tumor suppressors, as illustrated by the following evidence. TCGA analysis of 958 breast cancer patients found that KMT2C and KMT2D were altered in $6.99 \%$ and $2.40 \%$ of cases, respectively, and that $\mathrm{KMT} 2 \mathrm{C}$ copy number amplification (CNA) significantly associates with survival, though mRNA expression did not (L. Liu, Kimball, Liu, Holowatyj, \& Yang, 2015). In hormone receptor positive breast cancer (HRPBC) KMT2C mutation is a negative prognostic indicator: death HR:3.25 ( $p<0.0001)$. In this setting, it's inactivation may contribute to hormone therapy resistance, as HRPBC cell lines containing a specific KMT2C mutation could become estrogen independent in vitro (Manso et al., 2016). Both KMT2C (19.7\%) and KMT2D (26.0\%) are highly mutated in muscle-invasive bladder cancer (MIBC) according to TCGA data, and their vulnerability is replicated in an aggressive carcinogen-induced mouse model (BBN), although how these mutations contribute to tumorigenesis is still speculative (Fantini et al., 2018). Some mutations in KMT2D caused it to be excluded from the nucleus, perhaps precluding it's 
epigenetic function (Fantini et al., 2018). On a related note, homozygous mutation of the KMT2C SET domain in a mouse model caused early onset of ureter epithelial tumors, which were further exacerbated by hemizygous deletion of $\mathrm{p} 53$. These urothelial cells also showed marks of elevated DNA damage prompting the idea that $\mathrm{KMT} 2 \mathrm{C}$ and $\mathrm{KMT2D}$, along with their associated epigenetic complex, may serve as important coactivators of p53-mediated DNA repair (J. Lee et al., 2009). In cutaneous squamous cell carcinomas (cSCC), a study comparing exomes of 12 metastatic tumors determined KMT2D mutation was significantly enriched in metastatic tumors compared with primary tumors, with mutation rates of $62 \%$ compared to 31\%, respectively (Yilmaz et al., 2017). Whole-exome sequencing also revealed KMT2C to be highly altered in osteosarcoma, where it appears to play a role in migration and invasion (Chiappetta et al., 2017). Examples can also be found in non-solid tumors, with Follicular lymphoma (FL) and diffuse large B-cell lymphoma (DLBCL) showing somatic alteration frequencies in $\mathrm{KMT} 2 \mathrm{D}$ of $32 \%$ and $89 \%$, respectively, underscoring the widespread conserved role of these lysine methyltransferases as apparent tumor suppressors (Morin et al., 2011). In lung cancer, KMT2D positively regulates the super-enhancer of the circadian rhythm regulator per2. Loss of KMT2D in this context causes a reliance on glycolytic metabolism (Alam et al., 2020). This data comports well with previous data from our lab indicating that GRHL2 (likely collaborating with KMT2D ) represses the oncogenic glycolytic switch (Farris et al., 2016).

Our data provides another line of evidence for understanding the implications of KMT2C/D function in epithelial cells during tumorigenesis. Our data suggests that loss of function of KMT2C/D could, in effect, mimic GRHL2 loss of function to a degree, because GRHL2's inability to interact with these modifiers renders it largely non-functional as far as 
epithelial gene expression is concerned. Is GRHL2 rendered dysfunctional, permitting EMT, in carcinomas that exhibit loss of function in KMT2C or KMT2D? Loss of KMT2C and KMT2D surely has other major implications depending on the cell type, and independent of GRHL2 interaction, but it is worth noting that epithelial gene expression may also be dysregulated.

The process of EMT is challenging to detect in whole tumor sequencing; the minority of cells that downregulate or lose GRHL2, concurrent with EMT, though perhaps contributing disproportionately to immune evasion and metastasis, would likely be missed by a bulk approach to sequencing tumors. Further, data presented in Chapter II suggests that other consequential mechanisms of GRHL2 downregulation may be at play in the tumor microenvironment. In Chapter II, we investigate the importance of epithelial phenotype, as controlled by GRHL2, in reception and transmission of the interferon response: a critical node in innate and adaptive immunity.

\section{Chapter II. GRHL2 Promotes Interferon Signaling in Epithelial Cells.}

\section{Chapter II: Introduction}

Following on data presented in Chapter I describing the ability of GRHL2 to promote expression of the interferon stimulated gene (ISG) ICAM-1, we sought to uncover the breadth of GRHL2's regulation of interferon stimulated genes. We explore epithelial response to doublestrand RNA (dsRNA), and interferon gamma (IFN- - ): probing the importance of GRHL2 in relaying these signals, as well as the effect of their signaling on GRHL2 expression, itself. IFN- $\nu$ 
has previously been shown to induce EMT in cells, however, GRHL2's involvement in this mechanism was not directly implicated (D. Imai et al., 2019; M. Lee et al., 2019; Lv et al., 2015).

The interferon pathway is a conserved signaling pathway utilized by most lineages of human cells. Interferon signaling can be generally broken down into three categories based upon distinct receptors: Type I, II \& III. These responses are ignited through activation of pattern recognition receptors (PRRs) and include many pathogen associated molecular patterns such as double stranded RNA (dsRNA) which signals via toll-like receptor 3 (TLR3) or RIGI/MDA5, as well as many other ligands including single strand RNA (ssRNA), CpG DNA, and bacterial components (Abe, Marutani, \& Shoji, 2019; Schneider, Chevillotte, \& Rice, 2014). Although arising from distinct ligands, Type I and Type II signaling exhibits downstream crosstalk through signal transduction intermediates: some ligand-specific, others shared and overlapping.

The Type II interferon pathway (IFN-II) is predominantly mediated through IFN- - . IFN- $-\gamma$ is mainly derived from CD4+ T cells, CD8+ T cells, and NK cells in the tumor microenvironment, and is not thought to be produced by non-immune lineage cell types. It signals through homotypic interaction between ligand-bound IFNGR on the membrane, which initiates intracellular JAK1,2/STAT1 signaling. Phosphorylated STAT1 goes to the nucleus and recognizes "Gamma Activation Site (GAS)" motifs in interferon stimulated genes (ISGs), inducing their activation (Schneider et al., 2014).

As a core component of innate immunity, Type I interferon (IFN-I) constitutes an intrinsic first line of defense against viral infection in cells. Generally, and of initial interest to 
our research, dsRNA gets internalized into cells and may activate endosomal TLR3 or cytosolic MDA5/RIG-I (Sabelo Lukhele, Giselle M. Boukhaled, \& David G. Brooks, 2019). Activation of stimulator of IFN genes (STING) and mitochondrial antiviral-signaling protein (MAVS) promotes phosphorylation and subsequent nuclear localization of IRF3 and IRF7. Nuclear import of these IRF proteins allow them to localize to "Interferon Stimulated Response Element" (ISRE) motifs in regulatory regions of their target genes. This allows for quick transcription of a limited number of ISGs, including the very consequential Type I IFNs ( IFNß) (Schneider et al., 2014). Production of these factors provides a positive feedback autocrine signal to reinforce the antiviral state of the cell; slowing protein synthesis, upregulating major histocompatibility complex (MHC), and degrading viral RNA, while also signaling in a paracrine fashion, spreading an antiviral posture to its neighboring cells. Production of Type I IFN provides a ligand for the cell surface receptors IFNAR1 and IFNAR2, which activate associated JAK/STAT kinases, subsequently forming complexes of phospho-STAT1\&2 and IRF9 termed "ISGF3". This complex goes to the nucleus and further activates a wave of ISGs (Sabelo Lukhele et al., 2019; Snell, McGaha, \& Brooks, 2017).

In addition to engendering antiviral responses, Type I IFN production also has a welldocumented tumoricidal role. Its signaling can implement growth arrest and senescence, but also caspase activation and apoptosis (Apelbaum, Yarden, Warszawski, Harari, \& Schreiber, 2012; Besch et al., 2009). In the tumor setting, Type I IFN is likely predominantly triggered by DNA sensing PRRs activated by surrounding cell death. Treatment of human mesenchymal stem cells with IFN- $\alpha$ initiated a senescent phenotype in a dose dependent manner, in the absence of significant apoptosis. This IFN- $\alpha$ induced senescence was reliant upon nuclear accumulation of 
promyelocytic (PML) protein aggregates in the nucleus, which were subsequently shown to colocalized with p53 (Fu et al., 2015). Intriguingly, it has been shown that spontaneously immortalizing Li Fraumani fibroblasts downregulate IRF5 and IRF7 (IRF7 through CpG methylation), but that senescing fibroblasts reactivate these factors; IRF5 and IRF7 transfection was sufficient to slow growth and induce a senescence phenotype in immortalized fibroblasts coincident with activation of other ISGs (Q. Li et al., 2008). In support of this association, druginduced senescence upon administration of the genotoxic compounds 5-bromo-2'-deoxyuridin ( $\mathrm{BrdU}$ ) or distamycin $\mathrm{A}$ (DMA) in HeLa cells caused induction of IFN- $\beta$ and, by day six, $>5$-fold induction of 22 ISGs (Novakova et al., 2010). A multitude of Type I IFN response pathways relevant to tumor biology exist, including inflammation, generation of an antiviral state, apoptosis, pyroptosis, growth arrest and senescence.

Type I IFN is also critical for various stages of the adaptive immune response. CD4(+) Treg cells show numerical reduction in colorectal and melanoma models in response to IFN $\alpha$ treatment, however Type I IFN signaling has also been shown to increase immunosuppressive IDO and PD-1 expression on Tregs (Snell et al., 2017; Yu, Huang, Zong, He, \& Mo, 2016). Maintaining CD8(+) T cell fitness in both viral infections and tumor scenarios is critical; Type I IFN indirectly enhances their function by promoting dendritic cell (DC) cross-presentation and directly promotes their survival and cytotoxicity (Diamond et al., 2011). The tumor microenvironment actively modulates interferon receptor on CD8 T cells, downregulating its expression to quell robust antitumor immunity. Colorectal cancer mouse models in which IFNAR1-stabilized T-cells were adoptively transferred showed significantly improved anti-tumor effects compared with wildtype T-cell transfer (Katlinski et al., 2017). IFN-I signaling can also 
skew the population of CD8+ T cells towards a more cytotoxic phenotype, but with decreased renewal capacity. IFNR blockade reversed this trend and allows for emergence of a memory, TCF-1(+)CXCR5+ phenotype that is thought to be critical for effective, sustained response to a chronic viral infection (Snell et al., 2017) (Wiesel et al., 2012).

Notable cases from other kingdoms of life suggest the untapped potential of innate immunity pathways in controlling viral infection or tumorigenesis. The Pacific Oyster, Crassostrea gigas, can be induced to enter a prolonged "innate immune primed" state by transient treatment with the double-strand RNA mimetic poly $(\mathrm{I}: \mathrm{C})$. This artificial viral mimic induced an antiviral state that lasted up to 4 months post-priming, and was partly characterized by gene expression representing gene ontology (GO) categories such as "regulation of cell death", "regulation of type I interferon production" and "immune response". Poly(I:C) regulated genes sustained their differential expression patterns from day 1 - day 126 postpriming, though some regression to control was observed. Primed oyster populations maintained $100 \%$ survival 10 days post-infection with OsHV-1, while sterile filtered seawater control oyster populations had $5 \%$ survival at 10 days. The survival advantage of primed oysters coincided with a 200x lower viral DNA load, indicating ineffective viral replication after innate immune priming compared with the non-primed control (Lafont et al., 2020).

In a marine vertebrate model, Zebrafish danio rerio were depleted of adaptive immunity by homozygous deletion of $\mathrm{rag}$, then compared to $\mathrm{rag}^{+/+}$zebrafish response upon challenge with a lethal dose of spring viremia carp virus (SVCV). Rag $^{-/-}$fish exhibited an "antiviral alert state", with $40-45 \%$ survival compared to $0 \% \mathrm{rag}^{+/+}$survival 12 days post-infection. $\mathrm{Rag}^{-/-}$fish 
exhibited a 100x lower viral load compared to $\mathrm{rag}^{+/+}$. RNAseq analysis showed an increase in genesets corresponding to interferon, apoptotic, and multigene signatures comparing uninfected $\mathrm{rag}^{-/-}$versus uninfected $\mathrm{rag}^{+/+}$. $\mathrm{Rag}^{-/-}$fish also exhibited increased active Caspase-3

IHC staining in tissue. This suggests that some species may activate an antiviral primed state that can be maintained long-term and represents a robust response to infection in the absence of the adaptive arm of the immune system. Intriguingly, these fish are maintained in nonsterile conditions, possibly exposed to a microbiome capable of stimulating this low-level innate immunity (García-Valtanen et al., 2017) .

The Blind Mole Rat (genus Spalax) is a subterranean, long-lived rodent perhaps best known for its negligible rates of cancer occurrence despite a relatively long lifespan compared to other rodent species. Cancer mortality can account for up to $90 \%$ of some rat and mouse species, however, tumor development has never been documented in captive Blind Mole Rats. In vitro studies using fibroblast cell lines from Spalax judaei and Spalax golani demonstrated a novel tumoricidal pathway reliant on Type I interferon secretion. Fibroblast cell cultures underwent 7 20 population doubling, ceased to grow, then initiated massive necrotic cell death, resulting in "concerted cell death" of the entire population. The factor responsible for this cell death was demonstrated to be IFN- $\beta$ on the basis of GFP(+) viral infectivity assay in HT1080 cells, as well as a HEK-blue cell $\beta$-galactosidase promoter assay containing an IFN- $\beta$ responsive promoter that was sensitive to conditioned media from different population growth stages of Blind Mole Rat cells. The necrotic cell death response was dependent upon p53 and Rb pathways, as introduction of SV40 large T antigen abrogated cell death and IFN- $\beta$. This remarkable example 
of a species-specific innate immune response underscores the adaptability and tumoricidal potential of the innate arm of the immune system (Gorbunova V, Hine C, \& X, 2012).

These examples from across the Animal Kingdoms showcase the importance and effectiveness of first-line innate immunity. Functional and responsive interferon pathways, particularly the production of Type I IFNs, are equally critical in Homo sapiens; though perhaps not possessing identical mechanisms as Danio rerio or Spalax judaei, dissecting factors that regulate the timing, sensitivity and magnitude of this response in Homo sapiens is a priority.

In light of these observations, the epithelial production of Type I IFN emerges as a critical node in both innate and adaptive arms of anti-tumor and anti-viral immunity. As reviewed above, modulation of IFN production, receptor expression and signaling have implications for tumor progression and resolution. Identifying factors that maintain, or are necessary for, the IFN response could inform future understanding of the regulation of this process and identify predictors of response to immunotherapies. It is important to note that IFN signaling is often two-sided, capable of initiating robust immunity, but also immunosuppression in chronic settings. For example, expression of checkpoint molecules is a normal physiological response to avoid autoimmunity, but in the tumor microenvironment setting these molecules are associated with poor outcomes, while also predicting response to checkpoint blockade therapies (D. S. Chen \& Mellman, 2017).

The ramifications of epithelial versus mesenchymal state, or EMT, upon normal epithelial IFN I response is not well documented. During embryogenesis, the human embryonic stem cell (hESC) constitutively expresses a subset of interferon response genes in the absence 
of stimulation. These "Intrinsic Interferon Genes" are thought to protect the developing embryo from viral pathogens during formative development. Upon differentiation, the genes lose their constitutive expression, and the ubiquitous, stimulation-dependent interferon response pathway becomes accessible (X. Wu et al., 2018). This intrinsic ISG phenomenon and GRHL2's premier role in establishing epithelial enhancers upon hESC differentiation (A. F. Chen et al., 2018) converge upon a possible role for GRHL2 in regulating interferon response programs in differentiated epithelial lineages. In airway epithelial cells a TGF- $\beta$-induced EMT phenotype downregulated IFN I and IFN III expression. Zeb1 mediated repression of IRF1 and restrictive epigenetic remodeling, in this context, led to silencing of IFN III contributing, pathologically, to defective mucosal antiviral immunity (J. Yang, Tian, Sun, Garofalo, \& Brasier, 2017). In triple negative breast cancer (TNBC), an IFN- $\beta$ metagene signature predicts better prognosis and immune response. Treatment of drug resistant tumor cancer stem cell (CSC) populations, shown to lack IFN/STAT signature, with IFN- $\beta$ was able to revert CSC traits and induce epithelial traits (Doherty et al., 2017).

Speculatively, the extent to which EMT or hybrid EMT/MET could modulate the interferon response might have important implications for survival of these cells at primary tumor sites, as well as during metastatic dissemination, possibly constituting a survival advantage for the individual cell, and altering the immune microenvironment at the population level. In breast cancer, high expression of an interferon metagene predicts metastasis in ESR1+/ERBB2- tumors, however in ERBB2+ subtype it correlates with low metastasis (Callari et al., 2014). This suggests that extrinsic or intrinsic factors influencing interferon signaling could have a significant role in tumor biology and progression. 
We hypothesized that GRHL2 would preserve appropriate IFN response. Stated differently, loss of GRHL2 would reduce sensitivity to IFN- $\gamma$ and dsRNA and deregulate the cellular interferon response. We conducted whole transcriptome RNAseq on Mcf10a cells treated with these ligands in the presence or absence of GRHL2. The results suggest that GRHL2 regulates a multitude of ISGs, and, particularly, is required for Type I (IFN $\beta$ ) and Type III (IFNL1,2,3) production. These results provide exciting insights regarding the significance of the epithelial versus mesenchymal state for Type I IFN regulation, with implications for antiviral and antitumor immunity.

\section{Chapter II: Results}

II.a. GRHL2 regulates subset of interferon response genes (ISGs).

We probed a number of interferon stimulated genes basally and after IFN- $\gamma$ stimulation in Mcf10aneoT+TGF-b (+GRHL2) and HT1080 (+GRHL2). The results indicate that GRHL2 promotes expression of many interferon stimulated genes, but may also repress subsets

(Fig.10,a) (Table 2). Interestingly, GRHL2-expressing cells repressed expression of the top "intrinsic interferon genes" conserved across multiple hiPSC and hESC cell lines (Rice, et al. 2017), suggesting that GRHL2 may play a role in repressing this subset during transition from pluri- and multipotent stem cells into differentiated epithelial lineages (Fig. 10,b). This seems paradoxical because expression analysis of

\begin{tabular}{|ll|}
\hline \multicolumn{2}{|c|}{ Table 2} \\
\hline \multicolumn{2}{|c|}{ Effect of GRHL2 } \\
\multicolumn{2}{|c|}{ on ISGs } \\
\hline Induced & Repressed \\
\hline IFNLR & ISG15 \\
IFIH1 & OASL \\
DDX58 & OAS1 \\
PDL1 & BST2 \\
ICAM1 & EIFL3 \\
CD155 & IFITM1 \\
CXCL10 & IFITM3 \\
CXCL16 & \\
RNL & \\
P21 & \\
SOCS & \\
MLT1b & \\
TRIMM22 & \\
\hline
\end{tabular}


GRHL2 shows high expression in hESCs (with high intrinsic ISG activity), so our prediction of GRHL2 promoting intrinsic ISG expression was not supported.

A.
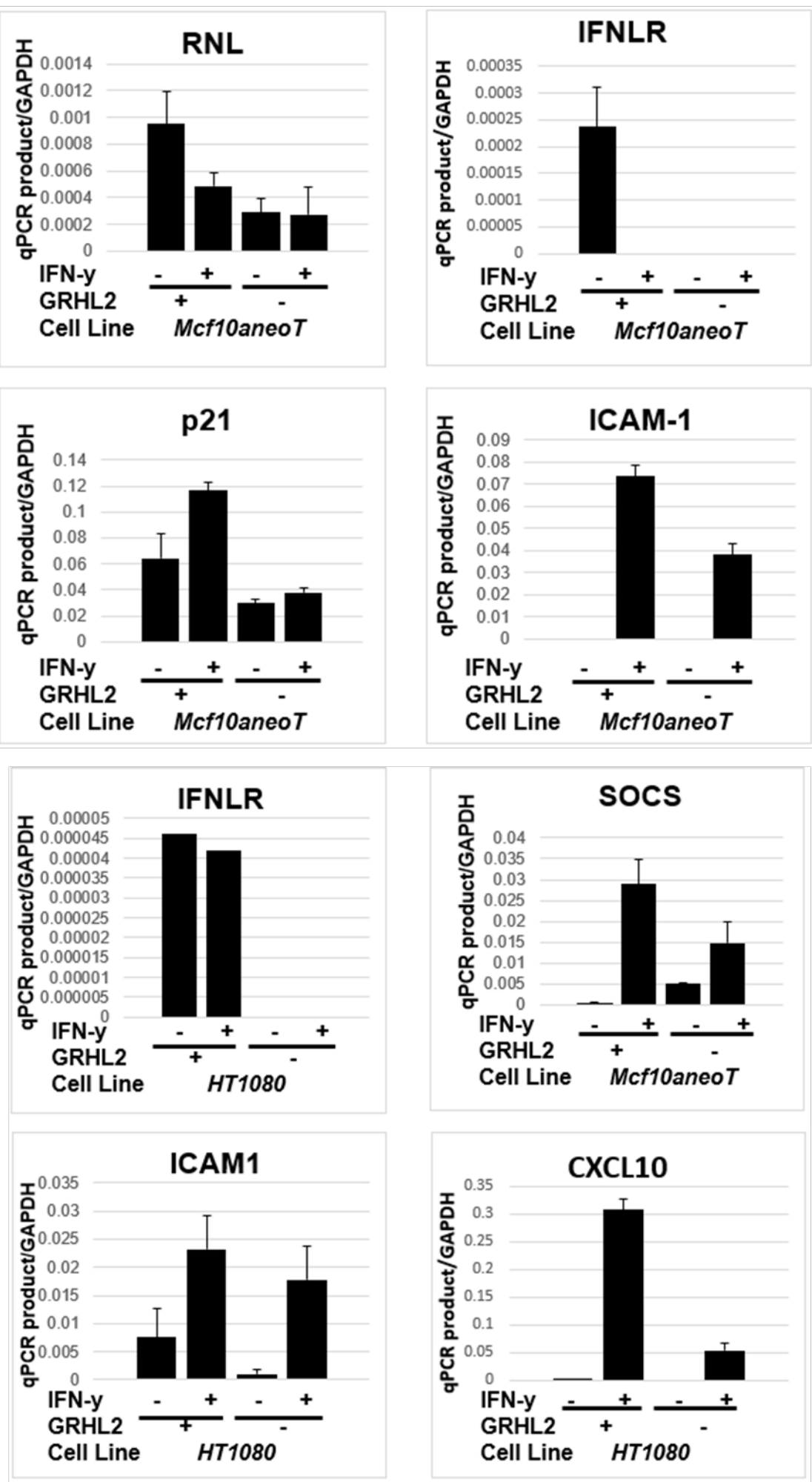
B.

\begin{tabular}{|c|c|}
\hline 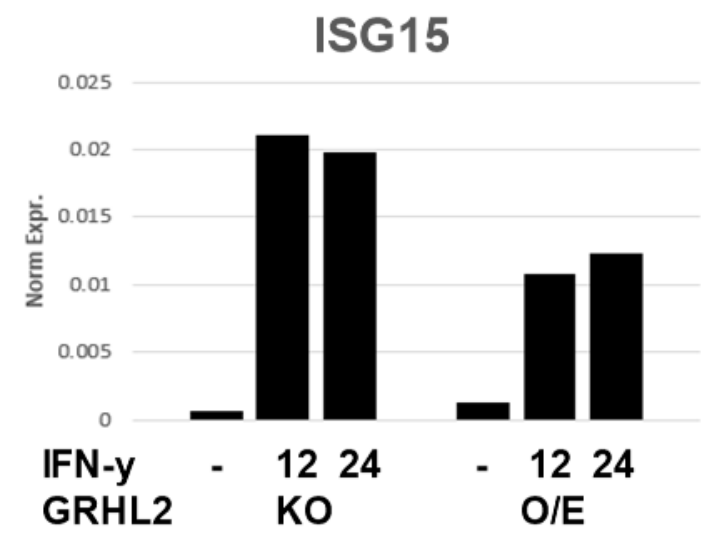 & 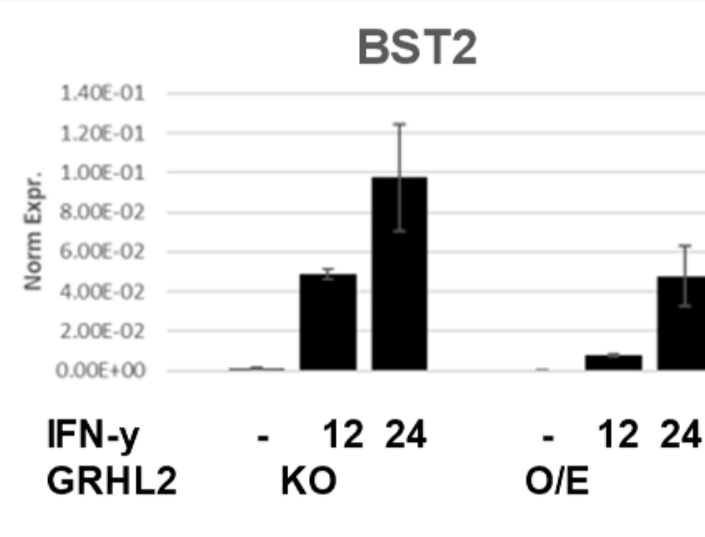 \\
\hline 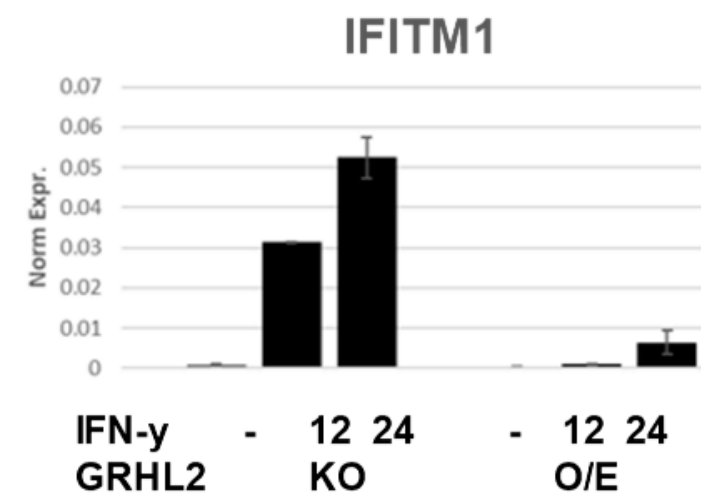 & 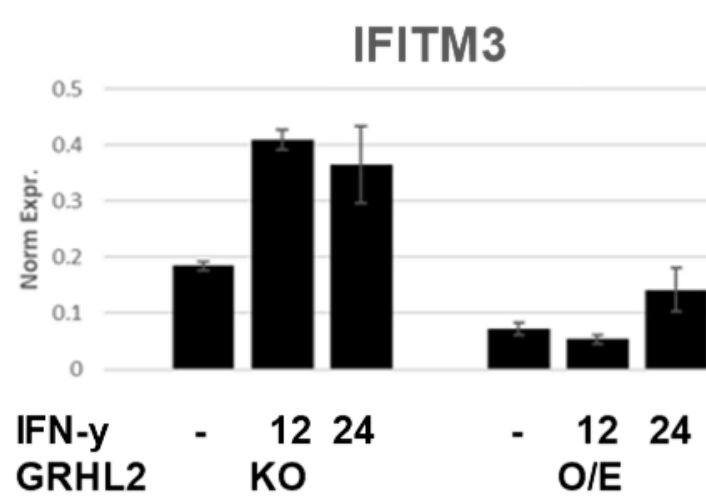 \\
\hline
\end{tabular}

Figure 10. mRNA transcript levels of select ISGs in Mcf10aneoT and HT1080. A.) GRHL2 expression favors ISG induction at basal and/or after IFN- $\gamma$ stimulation in Mcf10aneoT+TGF-B cells expressing ectopic GRHL2. Similar results were obtained for favorable ISG MRNA levels in qPCRs conducted in HT1080 fibrosarcoma cells ectopically expressing GRHL2. C.) A panel of "intrinsic" embryonal ISGs are repressed by GRHL2 expression in Mcf10aneoT cells ectopically expressing GRHL2 compared with CRISPR-Cas9 GRHL2 knockout cells. All qPCR results represent at least biological duplicates and are normalized to GAPDH or CBX housekeeping gene controls. 


\section{II.b. GRHL2 is required for Type I and III IFN production in Mcf10aneoT cells.}

Based upon differential targeted qPCR expression of various ISGs in the presence or absence of GRHL2, we wanted to know if GRHL2's potentiation and promotion of interferon response was pervasive across large swaths of interferon stimulated genes. It is estimated that around $5 \%$ of the human coding genome is responsive to interferon stimulation (Schneider et al., 2014), making whole transcriptome RNAseq analysis a more comprehensive method for this investigation. In order to explore IFN-I and IFN-II pathways, we separately treated cells with either dsRNA mimetic poly(I:C) or recombinant IFN- $\gamma$ and compared with untreated control cells. Mcf10aneoT (GRHL2 WT), Mcf10aneoT+GRHL2 (O/E) , and Mcf10aneoT+gr sg2 (GRHL2 CRISPRcas9 K.O.) were treated with either [600U/mL] IFN- $\gamma$ for $12 \mathrm{hrs}$, or [50ug/mL] poly(I:C) for $3 \mathrm{hrs}$, in triplicate. To explore differences in ISG expression, we first calculated the fold induction of the top 100 most highly upregulated genes in our GRHL2 WT cells after poly(l:C) treatment. We compared the induction of these genes with the corresponding induction in GRHL2 K.O. cells. Analysis revealed that many of the genes upregulated in WT cells were classical ISGs, and that these genes exhibited weaker induction, or no expression, in our GRHL2 K.O. cells (Fig. 11,a). Strikingly, interferon beta (IFNB1) and interferon lambda 1, 2, and 3 (IFNL1, IFNL2, IFNL3), definitive members of Type I and Type III Interferons, respectively, were either marginally, or not expressed in the GRHL2 knockout cells after poly(I:C) stimulation (Fig. 11,C). 
A.

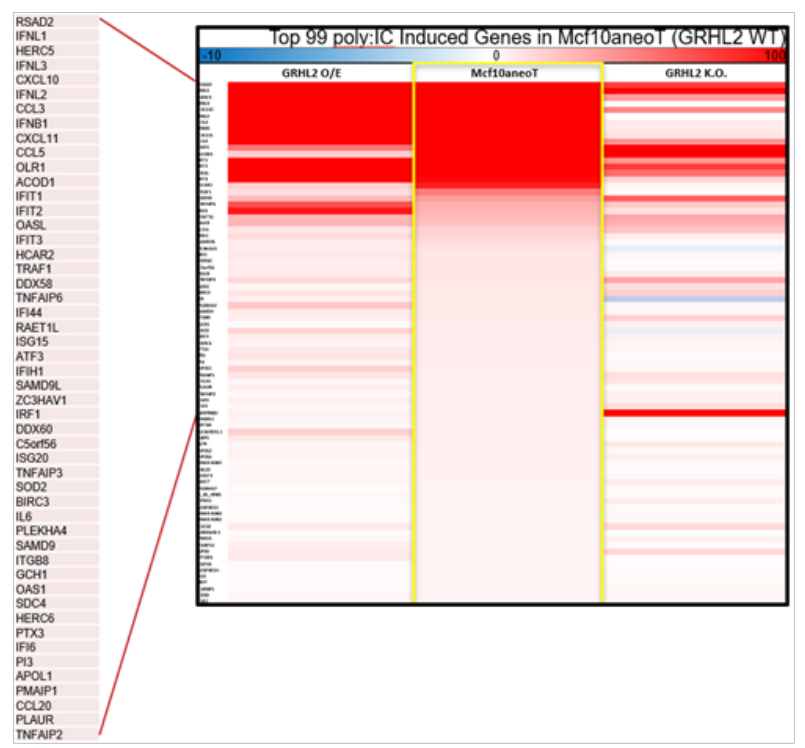

B.

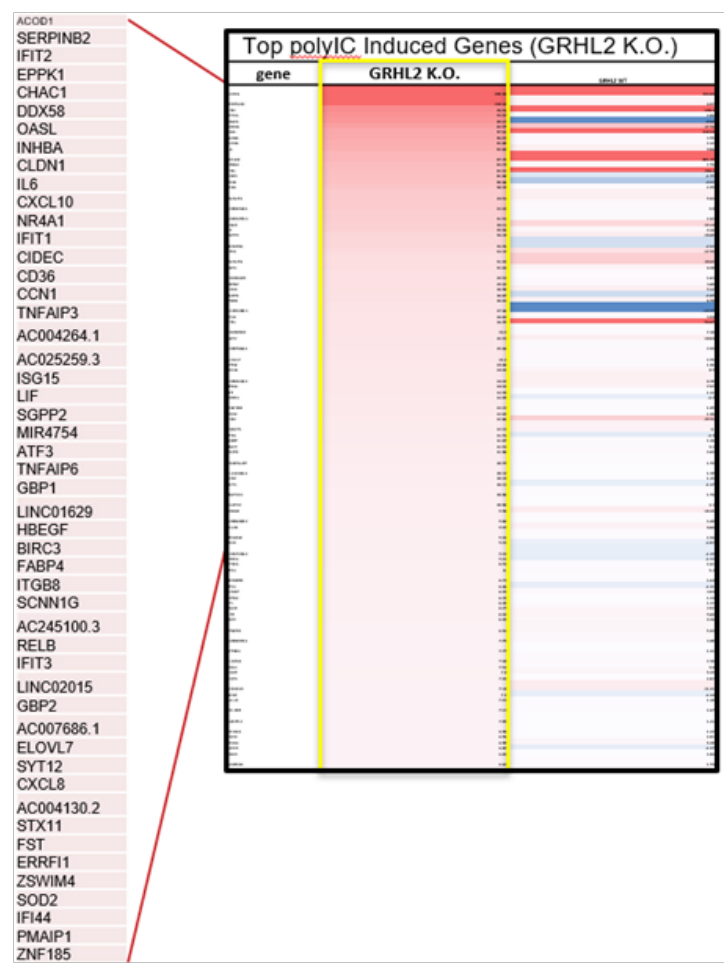

C.

\begin{tabular}{|l|rrrrrr||}
\hline & \multicolumn{5}{c|}{ Interferon Signaling and Response Genes (FPKM) } \\
\hline (+ poly:IC) & \multicolumn{4}{c|}{ Mcf10aneoT GRHL2 ${ }^{+++}$} & \multicolumn{3}{c|}{ Mcf10aneoT GRHL2 } \\
Gene & sample 1 & sample 2 & sample 3 & sample 4 & sample 5 & sample 6 \\
\hline IFNL3 & 38.39883 & 38.69247 & 38.76367 & 0 & 0 & 0 \\
IFNL2 & 42.23914 & 42.52932 & 41.34321 & 0 & 0 & 0 \\
RSAD2 & 41.789 & 37.99318 & 33.99278 & 0.183871 & 0.246288 & 0.553915 \\
\hline IFNB1 & 15.75027 & 21.95654 & 19.1142 & 0.20697 & 0 & 0.406631 \\
CXCL11 & 57.80399 & 50.14781 & 47.7559 & 2.002228 & 1.422223 & 1.549661 \\
CXCL10 & 131.7122 & 119.4563 & 107.5738 & 7.241483 & 5.168766 & 6.774883 \\
IRF1 & 51.67111 & 53.20098 & 54.22579 & 11.09578 & 11.89521 & 13.9337 \\
\hline IRF7 & 6.81038 & 5.526335 & 5.358256 & 1.462577 & 1.583083 & 2.655818 \\
IRF9 & 0.703297 & 0.507285 & 0.461115 & 0.091538 & 0.136235 & 0.329714 \\
\hline IRF2 & 13.20288 & 13.38918 & 15.62367 & 5.882532 & 7.576367 & 7.408551 \\
IRF6 & 16.23104 & 16.17831 & 17.30349 & 0 & 0.051989 & 0.022877 \\
\hline STAT2 & 9.901752 & 8.605998 & 8.44439 & 3.586144 & 3.81528 & 4.109965 \\
STAT1 & 39.94704 & 36.33878 & 35.50195 & 26.04861 & 23.84566 & 24.16083 \\
\hline JAK2 & 9.576003 & 7.709248 & 7.821107 & 2.589238 & 2.094314 & 3.262344 \\
\hline IFIT3 & 426.1618 & 399.8509 & 385.9938 & 57.01562 & 52.20719 & 63.2482 \\
\hline IFIT1 & 100.0746 & 92.00698 & 84.64875 & 10.23672 & 7.449565 & 9.61233 \\
IFIT2 & 176.1842 & 171.9271 & 168.6918 & 36.22991 & 31.46426 & 40.81013 \\
\hline IFIT5 & 19.71057 & 17.81125 & 20.37213 & 8.662996 & 8.050138 & 9.455602 \\
\hline Color Code & 60 & & & 30 & & 0 \\
\hline
\end{tabular}


Figure 11. GRHL2 broadly promotes poly(l:C) induced antiviral interferon gene expression. Cells were treated in triplicate with $50 \mathrm{ug} / \mathrm{mL}$ poly $(\mathrm{l}: \mathrm{C})$ in Mcf10a media for $3 \mathrm{hrs,}$ before RNA prep. A.) RNAseq datasets comparing Mcf10aneoT (GRHL2 O/E, WT, K.O.) were used to calculate the most strongly induced genes (ranked by fold change between (-)poly(I:C) and (+)poly(l:C)) in GRHL2 WT (middle column). B.) Corresponding fold change is compared in GRHL2 O/E (left column) and K.O. (right column). Fold induction calculations used the average of triplicates per treatment group. C.) Representative classic interferon response genes (Type I and III IFN, IFITS, JAK/STAT, CXCLS) FPKM expression values in triplicate, after poly(I:C) treatment comparing Mcf10aneoT WT (left) and K.O (right).

Conversely, we took the top 99 genes most strongly induced in the K.O. cells after poly $(\mathrm{I}: \mathrm{C})$ and compared their induction to the same genes in WT (Fig. 11,b). This revealed a set of genes that were induced by poly(I:C) more strongly in the absence of GRHL2 (an EMT phenotype) and may constitute an interferon response signature that GRHL2 represses.

To find unifying pathways or processes from the most highly expressed genes, we took the absolute expression (FPKM) after poly(I:C) treatment of all genes in the RNAseq comparing WT and K.O.. (sample exhibited in Fig. 11,c) We used the final expression level after treatment to attempt selection for more biologically relevant ISGs based on higher expression, while avoiding artifacts arising from selection of genes with large induction fold changes, but extremely low expression values before and after treatment. We submitted these lists to Gene Set Enrichment Analysis (GSEA, Broad Institute), querying the gene list against a group of Gene Sets from Molecular Signature Database (www.gsea-msigdb.org) that were affiliated with the term "interferon". We found that our GRHL2 WT+poly(I:C) samples were disproportionately 
enriched for genes contributing to many dsRNA and interferon response gene sets with false discovery rates (FDR) $<0.25$ (Fig. 12, a,c). Conversely, the GRHL2 K.O.+poly(I:C) samples were nearly devoid of enrichment in these gene sets (Fig. 12, b). To investigate the genes from our GRHL2 WT+poly(I:C) dataset that were most influential on its association with interferon GSEA signatures, we conducted Leading Edge Analysis on enriched genesets from our original GSEA with an FDR $<0.25$ and a NES $>1.20$. This analysis quantifies the number of times that a gene appears across multiple gene sets. The top hits and number of appearances in the GRHL2 WT+poly(I:C) were IRF7, ISG15, TAP1 and IRF1 (Fig. 12, d). Our data suggests that GRHL2 promotes IFN-I signaling in epithelial cells. Loss of GRHL2 abated IFN-I and IFN-III production, indicating a breakdown in the signal transduction process somewhere between ligand reception and transcriptional activation of IFN-I and IFN-III. Apart from these specific cytokines, GRHL2 also was required for upregulation of numerous other ISGs, which together, contribute to enrichment of various GSEA pathways such as "Response to oncocytic virus", "IFN alpha response up", and "Graft versus host disease 35D up". 
A.

\section{"Interferon" Genesets Enriched in GRHL2 WT}

GENE SET

KUROZUMI_RESPONSE_TO_ONCOCYTIC_VIRUS

ICHIBA_GRAFT_VERSUS_HOST_DISEASE_35D_UP GSE35825_IFNA_VS_IFNG_STIM_MACROPHAGE_UP

GSE18791_CTRL_VS_NEWCASTLE_VIRUS_DC_14H_DN DER IFN_ALPHA RESPONSE_UP

GSE45365_WT_VS_IFNAR_KO_CD11B_DC_MCMV_INFECTION_DN

GSE40666_UNTREATED_VS_IFNA_STIM_STAT4_KO_EFFECTOR_CD8_TCEL_90MIN

ICHIBA_GRAFT_VERSUS_HOST_DISEASE_D7_UP

GSE1432_1H_VS_6H_IFNG_MICROGLIA_DN

GSE3337_CTRL_VS_4H_IFNG_IN_CD8POS_DC_DN

GSE1432_CTRL_VS_IFNG_1H_MICROGUA_DN

GO_NEGATIVE_REGULATION_OF_TYPE_I_INTERFERON_PRODUCTION

BROWNE_HCMV_INFECTION_6HR_UP

KEGG_RIG_I_UKE_RECEPTOR_SIGNAUNG_PATHWAY

DER_IFN_GAMMA_RESPONSE_UP

GSE1432_CTRL_VS_IFNG_6H_MICROGUA_DN

GSE18791_CTRL_VS_NEWCASTLE_VIRUS_DC_12H_DN

GSE1740_UNSTIM_VS_IFNA_STIMULATED_MCSF_DERIVED_MACROPHAGE_DN

GSE5542_IFNG_VS_IFNA_AND_IFNG_TREATED_EPITHELIAL_CEUSS_6H_UP

GSE7509_DC_VS_MONOCYTE_WITH_FCGRIIB_STIM_DN

GSE18791_CTRL_VS_NEWCASTLE_VIRUS_DC_16H_DN

GO_REGULATION_OF_TYPE_I_INTERFERON_MEDIATED_SIGNALING_PATHWAY

SIZE

\begin{tabular}{|l|l|}
\hline & NES \\
\hline 35 & 1.819462 \\
\hline
\end{tabular}

NOM p-val FDR q-val

\begin{tabular}{|r|r|r|r|}
\hline 125 & 1.766397 & 0 & 0.06611399 \\
\hline
\end{tabular}

\begin{tabular}{l|l|l|l|l|}
170 & 1.732503 & 0 & 0.101303995
\end{tabular}

\begin{tabular}{l|l|l|l|}
169 & 1.547627 & 0 & 0.24380837 \\
\hline
\end{tabular}

\begin{tabular}{l|l|l|l|}
73 & 1.546439 & 0 & 0.22573489 \\
\hline
\end{tabular}

\begin{tabular}{|l|l|l|l|}
157 & 1.544437 & 0 & 0.21067365 \\
\hline
\end{tabular}

\begin{tabular}{|l|l|l|l|}
\hline 193 & 1.542251 & 0 & 0.20117785 \\
\hline
\end{tabular}

\begin{tabular}{l|l|l|l|}
96 & 1.540452 & 0 & 0.1960548 \\
\hline
\end{tabular}

\begin{tabular}{|l|l|l|l|}
\hline 199 & 1.540126 & 0 & 0.18598446 \\
\hline
\end{tabular}

\begin{tabular}{l|l|l|l|}
185 & 1.539835 & 0 & 0.17717296 \\
\hline
\end{tabular}

\begin{tabular}{|l|l|l|l|}
182 & 1.538807 & 0 & 0.17250334 \\
\hline
\end{tabular}

\begin{tabular}{|l|l|l|l|}
41 & 1.532131 & 0 & 0.17011186 \\
\hline
\end{tabular}

\begin{tabular}{|l|l|l|l|}
53 & 1.525441 & 0 & 0.172417 \\
\hline
\end{tabular}

\begin{tabular}{|l|l|l|l|}
\hline 58 & 1.524805 & 0 & 0.1660462 \\
\hline
\end{tabular}

\begin{tabular}{lll|l|l|}
70 & 1.522116 & 0 & 0.16229291
\end{tabular}

\begin{tabular}{l|l|l|l|}
196 & 1.522046 & 0 & 0.15696147 \\
\hline
\end{tabular}

\begin{tabular}{l|l|l|l|}
188 & 1.517821 & 0 & 0.15392956 \\
\hline
\end{tabular}

\begin{tabular}{l|l|l|l|}
173 & 1.516145 & 0 & 0.14939094 \\
\hline
\end{tabular}

\begin{tabular}{|l|l|l|l|}
184 & 1.510519 & 0 & 0.14859344 \\
\hline
\end{tabular}

\begin{tabular}{l|l|l|l|}
190 & 1.507764 & 0 & 0.14460918 \\
\hline
\end{tabular}

\begin{tabular}{|l|l|l|l|}
\hline 181 & 1.498514 & 0 & 0.15654366 \\
\hline
\end{tabular}

\begin{tabular}{l|l|l|l|}
29 & 1.496708 & 0 & 0.15406834 \\
\hline
\end{tabular}

GSE1432_CTRL_VS_IFNG_24H_MICROGLIA_DN

GSE45365_HEALTHY_VS_MCMV_INFECTION_CD11B_DC_IFNAR_KO_DN

\begin{tabular}{l|l}
192 & 1.490365
\end{tabular}

$166 \quad 1.489349$

$0 \quad 0.15758783$

GO_RESPONSE_TO_TYPE_I_INTERFERON

GSE10325_MYELOID_VS_LUPUS_MYELOID_DN

GSE10325_BCELL_VS_LUPUS_BCELL_DN

GSE45365_WT_VS_IFNAR_KO_BCELL_DN

GSE45365_WT_VS_IFNAR_KO_CD11B_DC_UP

GSE22589_SIV_VS_HIV_AND_SIV_INFECTED_DC_UP

GSE45365_NK_CELL_VS_CD8A_DC_UP

GSE22589_HEALTHY_VS_HIV_INFECTED_DC_DN

HALLMARK_INTERFERON_ALPHA_RESPONSE

REACTOME_INTERFERON_ALPHA_BETA_SIGNALING

801.487997

$0 \quad 0.15383501$

\begin{tabular}{l|l|l|l|}
195 & 1.487235 & 0 & 0.14703284 \\
\hline
\end{tabular}

\begin{tabular}{|l|l|l|l|}
\hline 193 & 1.486676 & 0 & 0.1465001 \\
\hline
\end{tabular}

\begin{tabular}{|l|l|l|l|}
\hline 169 & 1.485093 & 0 & 0.1435149 \\
\hline
\end{tabular}

\begin{tabular}{l|l|l|l|}
185 & 1.480661 & 0 & 0.1419067 \\
\hline
\end{tabular}

\begin{tabular}{|l|r|r|r|}
175 & 1.4796 & 0 & 0.13921495 \\
\hline
\end{tabular}

\begin{tabular}{l|l|l|l|}
193 & 1.478559 & 0 & 0.13666852 \\
\hline
\end{tabular}

\begin{tabular}{l|l|l|l|l|l|}
188 & 1.477619 & 0 & 0.13425629 \\
\hline
\end{tabular}

\begin{tabular}{|r|r|r|r|}
\hline 97 & 1.476052 & 0 & 0.13196756 \\
\hline
\end{tabular}

571.472615

0.0 .13190486

B.

"Interferon" Genesets Enriched in GRHL2 K.O.

GENE SET $\quad$ SIZE

GSE45365_CD8A_DC_VS_CD11B_DC_UP

GSE3920_IFNA_VS_IFNG_TREATED_FIBROBLAST_DN

ICHIBA_GRAF_VERSUS_HOST_DISEASE_35D_DN

GSE10325_LUPUS_CD4_TCELL_VS_LUPUS_BCELL_DN

GSE45365_HEALTHY_VS_MCMV_INFECTION_CD8A_DC_DN

BROWNE_HCMV_INFECTION_12HR_DN

BROWNE_HCMV_INFECTION_1HR_DN

CHENG_RESPONSE_TO_NICKEL_ACETATE

GSE45365_HEALTHY_VS_MCMV_INFECTION_CD8A_DC_IFNAR_KO_DN

GSE3920_UNTREATED_VS_IFNA_TREATED_ENDOTHELIAL_CELL_UP

GSE3337_CTRL_VS_4H_IFNG_IN_CD8POS_DC_UP

ICHIBA_GRAFT_VERSUS_HOST_DISEASE_D7_DN

GSE45365_CD8A_DC_VS_CD11B_DC_IFNAR_KO_DN

GSE11864_UNTREATED_VS_CSF1_PAM3CYS_IN_MAC_DN

GSE3203_INFLUENZA_INF_VS_IFNB_TREATED_IN_BCELL_UP

BROWNE_HCMV_INFECTION_48HR_DN

GSE45365_NK_CELL_VS_BCELL_DN

GSE10325_LUPUS_BCELL_VS_LUPUS_MYELOID_DN

GSE7509_DC_VS_MONOCYTE_WITH_FCGRIIB_STIM_UP

GSE10325_CD4_TCELL_VS_MYELOID_DN

BOSCO_EPITHEUAL_DIFFERENTIATION_MODULE

GSE21774_CD62L_POS_CD56_DIM_VS_CD62L_NEG_CD56_DIM_NK_CELL_UP

BROWNE_HCMV_INFECTION_2HR_UP

GSE11864_UNTREATED_VS_CSF1_IFNG_PAM3CYS_IN_MAC_DN

GSE1740_MCSF_VS_MCSF_AND_IFNG_DAY2_DERIVED_MACROPHAGE_WITH_IFN

BROWNE_HCMV_INFECTION_18HR_DN

GSE45365_WT_VS_IFNAR_KO_CD8A_DC_DN

GSE45365_NK_CELL_VS_BCELL_UP

GSE11864_UNTREATED_VS_CSF1_IFNG_PAM3CYS_IN_MAC_UP

BROWNE_HCMV_INFECTION_1OHR_DN

GSE5542_UNTREATED_VS_IFNA_AND_IFNG_TREATED_EPITHEUAL_CELLS_24H_DI

GSE18791_CTRL_VS_NEWCASTLE_VIRUS_DC_1H_DN

GSE1432_6H_VS_24H_IFNG_MICROGLIA_UP

COLINA_TARGETS_OF_4EBP1_AND_4EBP2

\begin{tabular}{|r|r|r|r|}
\hline 183 & -1.79928 & NOM & F-val \\
\hline 186 & -1.75233 & 0 & 0.044999983 \\
\hline 43 & -1.7446 & 0 & 0.07460122 \\
\hline 166 & -1.73862 & 0 & 0.08446832 \\
\hline 188 & -1.73574 & 0 & 0.07460122 \\
\hline 96 & -1.73337 & 0 & 0.06868096 \\
\hline 195 & -1.7311 & 0 & 0.06473418 \\
\hline 43 & -1.72411 & 0 & 0.061915014 \\
\hline 194 & -1.71586 & 0 & 0.059800614 \\
\hline 184 & -1.69059 & 0 & 0.05815611 \\
\hline 184 & -1.6861 & 0 & 0.06276074 \\
\hline 36 & -1.68341 & 0 & 0.061146185 \\
\hline 178 & -1.67191 & 0 & 0.05980066 \\
\hline 181 & -1.66856 & 0 & 0.05866215 \\
\hline 181 & -1.66742 & 0 & 0.05768627 \\
\hline 433 & -1.66592 & 0 & 0.056840498 \\
\hline 131 & -1.66406 & 0 & 0.05610047 \\
\hline 189 & -1.66088 & 0 & 0.05544749 \\
\hline 197 & -1.65858 & 0 & 0.054867093 \\
\hline 184 & -1.65797 & 0 & 0.05434777 \\
\hline 58 & -1.64882 & 0 & 0.05388037 \\
\hline 192 & -1.64789 & 0 & 0.053457543 \\
\hline 35 & -1.64153 & 0 & 0.0530731 \\
\hline 183 & -1.63611 & 0 & 0.052722093 \\
\hline 198 & -1.63288 & 0 & 0.052400332 \\
\hline 161 & -1.62973 & 0 & 0.05210433 \\
\hline 194 & -1.62398 & 0 & 0.051831085 \\
\hline 194 & -1.61994 & 0 & 0.05157807 \\
\hline 190 & -1.61824 & 0 & 0.051343136 \\
\hline 53 & -1.61243 & 0 & 0.051124398 \\
\hline 184 & -1.61133 & 0 & 0.05092024 \\
\hline 155 & -1.60044 & 0 & 0.050729267 \\
\hline 196 & -1.59293 & 0 & 0.052400306 \\
\hline 336 & -1.59091 & 0 & 0.053904027 \\
\hline & & 0 & 0.055383388 \\
\hline
\end{tabular}




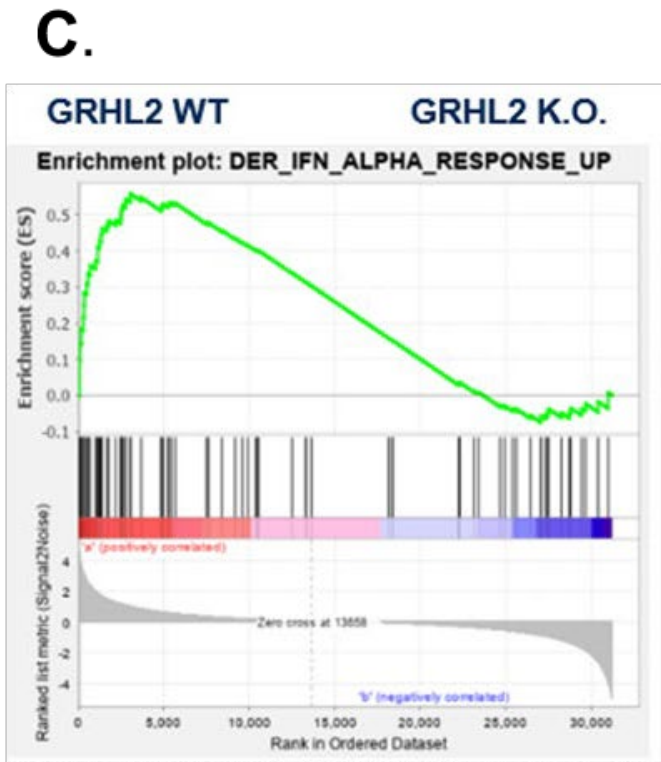

GRHL2 WT

GRHL2 K.O.

Enrichment plot: DER_IFN_ALPHA_RESPONSE_UP

Enrichment plot: DER_IFN_BETA_RESPONSE_UP
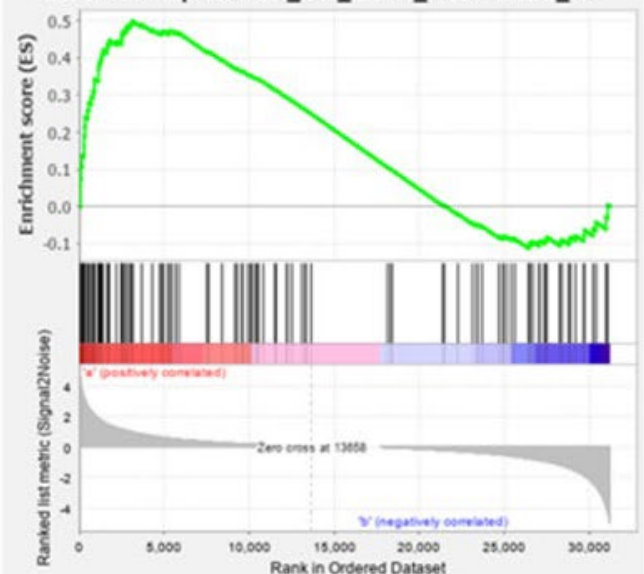

GO_NEGATIVE_REGULATION_OF_TYPE_I_INTERFERON KEGG_RIG_I_LIKE_RECEPTOR_SIGNALING_PATHWAY
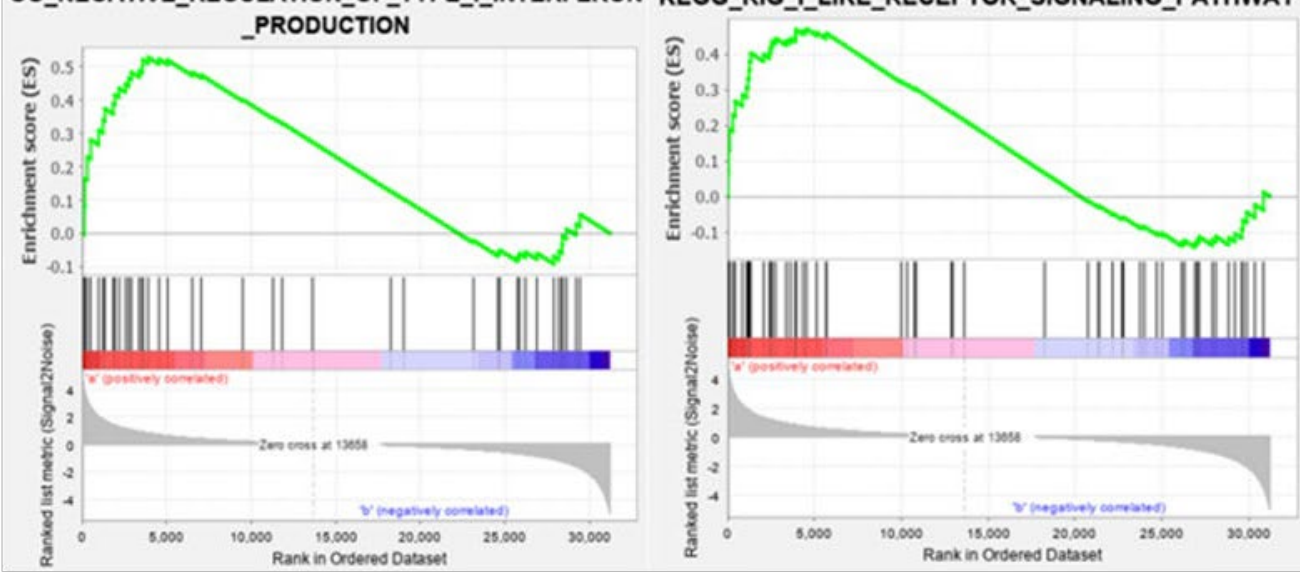

D.

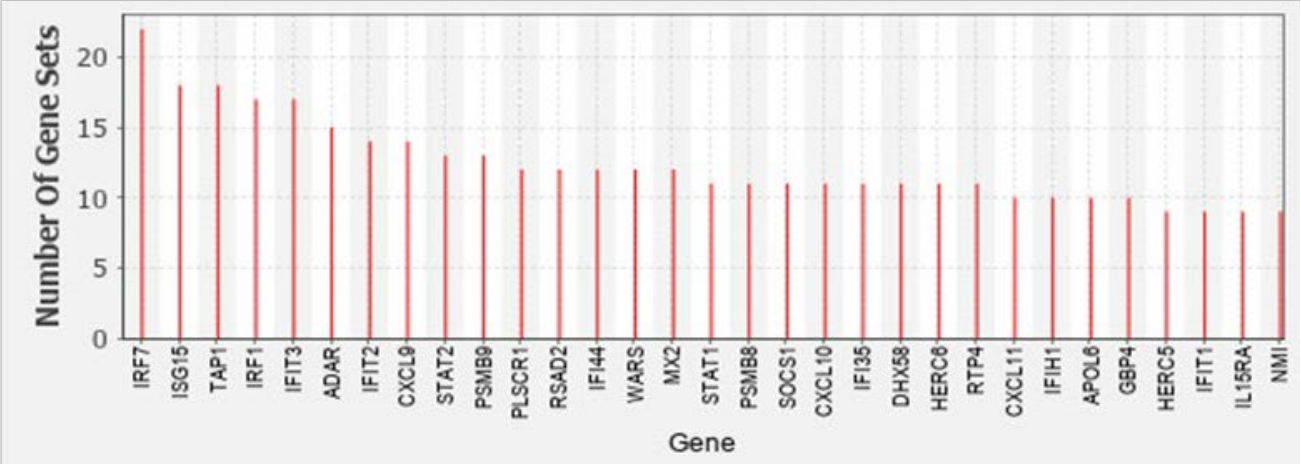


Figure 12. Gene Set Enrichment Analysis (GSEA) using Broad Institute Molecular Signature Database indicates enriched antiviral and IFN pathways in GRHL2 WT poly(I:C) treated cells. A.) RNAseq datasets for poly (I:C) treated Mcf10aneoT GRHL2 WT and GRHL2 K.O. were fed through GSEA software to compare enrichment for genes represented in multiple "Interferon" associated Gene Sets from Msigdatabase.org. A.) A list of the top "interferon" gene sets enriched in GRHL2 WT cells after poly(I:C) treatment. B.) A list of the top "interferon" gene sets enriched in GRHL2 K.O. cells after poly(I:C) treatment. C.) Representative GSEA enrichment plots. D.) Leading Edge Analysis conducted through the GSEA software package determined genes that most contributed to enrichment of GRHL2 WT in the gene sets from A).

\section{II.c. GRHL2 sensitizes cells to poly(I:C) and is downregulated by IFN- $\gamma$.}

Treatment of Mcf10aneoT (GRHL2 WT) cells with IFN- $-\gamma$ downregulated GRHL2 at 48hrs (Fig. 13, a) . We chronically treated Mcf10aneoT cells with IFN- $\gamma$ and observed a transition to a mesenchymal morphology. This led us to hypothesize that IFN- $\gamma$ could downregulate GRHL2, thus leading to an epithelial-mesenchymal transition. Western blot analysis of chronic IFN- $\gamma$ treated Mcf10aneoT cells revealed protein expressions consistent with an EMT (loss of GRHL2, E-cadherin/gain of $\mathrm{N}$-cadherin, vimentin, and fibronectin.) Interestingly, withdrawal of IFN- $\gamma$ could not rescue this EMT, suggesting that IFN- $\gamma$ had initiated stable repression of GRHL2 (Fig. $13, \mathrm{~b})$. In our first experiment we chronically treated the cells with IFN- $\gamma$ and made lysates when changes were observed. To repeat this phenomenon, we treated Mcf10aneoT cells with 400U/mL IFN- $\gamma$ and observed convincing EMT morphology in $~ 7$ days. We also conducted an NK 
assay on Mcf10aneoT versus Mcf10aneoT+ IFN- $\gamma$ and found that the latter had become significantly resistant to cell-mediated NK cytotoxicity. The assay was conducted in the absence of exogenous IFN- $\gamma$ treatment (Fig. 13, c).

Type I IFN may activate cell death pathways, inducing apoptosis, in some conditions (Apelbaum et al., 2012; Bernardo, Cosgaya, Aranda, \& Jiménez-Lara, 2013; Chawla-Sarkar M., 2001). On top of the widespread IFN gene expression augmentation regulated by GRHL2, we tested overall viability after transfection with $2 \mathrm{ng} / \mathrm{mL}$ poly $(\mathrm{l}: \mathrm{C})$. Interestingly, we found that GRHL2 WT and GRHL2 K.O. exhibited similar viability, while GRHL2 O/E cells were significantly more sensitive (Fig. 13, d). Various mechanisms of cell growth arrest and apoptosis in response to Type-I interferon signaling have been published. We could speculate that this sensitivity may be mediated through Type-I interferon upregulation of TRAIL (TNFSF10), stimulating death receptors on neighboring cells and inducing caspase-8 mediated apoptosis. Our RNAseq data hints that cells expressing GRHL2 (WT) start to upregulate TRAIL at 3hrs post poly(I:C) treatment, while GRHL2 K.O. cells, in contrast, completely turn off expression after poly(I:C) stimulation (Fig 13, e). This hypothesis warrants further investigation. 


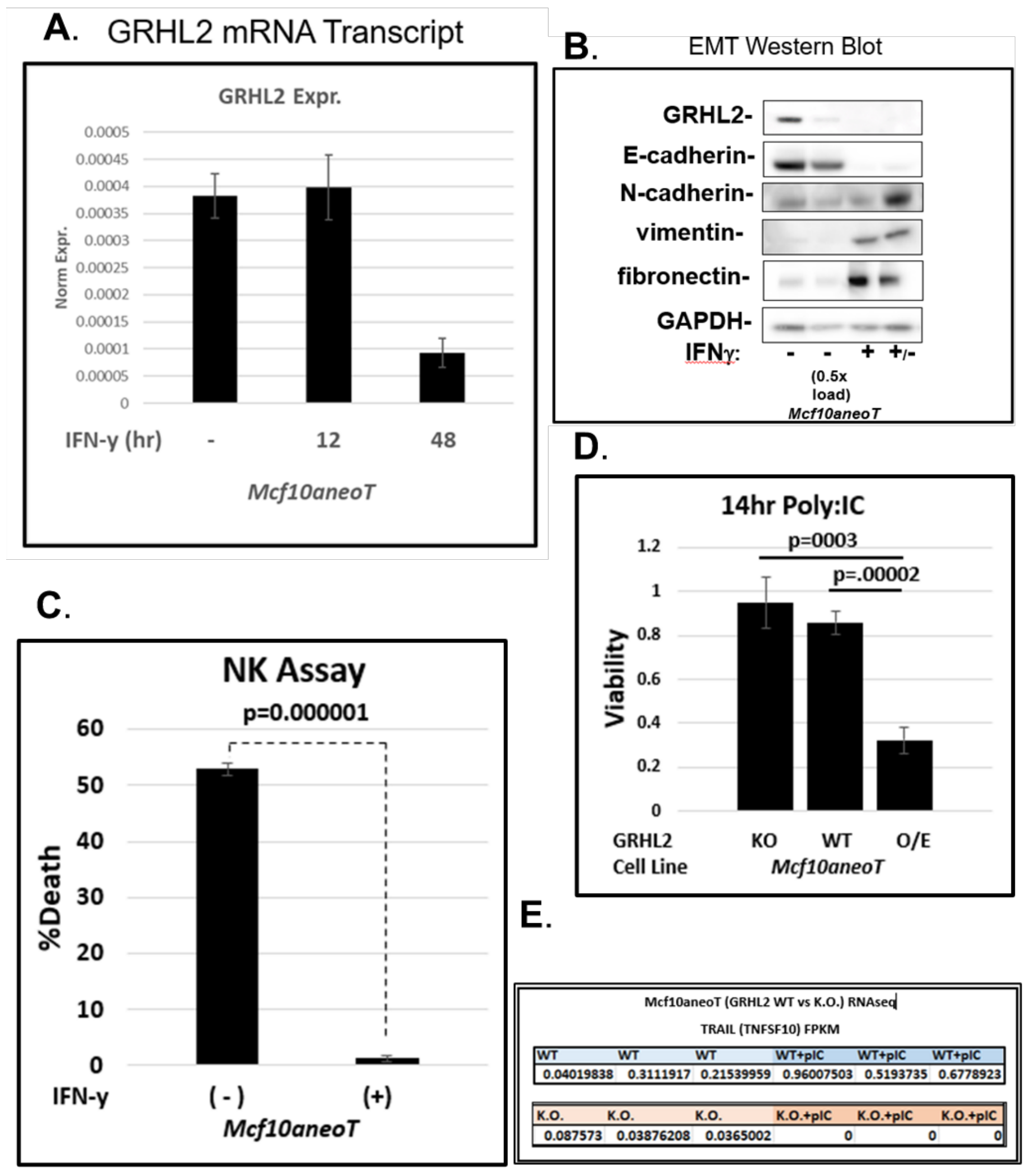


Figure 13. IFN- $\gamma$ downregulates GRHL2, thereby inducing epithelial to mesenchymal transition. A.) Normalized GRHL2 mRNA transcript levels decline within $48 \mathrm{hrs}$ of $\sim 400 \mathrm{U} / \mathrm{mL}$ IFN- $\gamma$ treatment. B.) Mcf10aneoT epithelial cells treated with chronic IFN- $\gamma[400 \mathrm{U} / \mathrm{mL}]$ undergo morphological changes consistent with EMT; western blot of cell lysates indicate loss of GRHL2 protein expression with accompanying E-cadherin loss and gain of $\mathrm{N}$-cadherin and Fibronectin expression. Lane 4 represents lysate from EMT'd cells that had IFN- $\gamma$ removed, suggesting a stable EMT was induced, even after withdrawal of the EMT stimulus. C.) Chronic IFN- $\gamma$ treated EMT cells were subjected to an NK assay (in the absence of IFN- $\gamma$ ). IFN- $\gamma$ induced EMT corresponded to significant resistance to NK attack compared to IFN- $\gamma$ naïve cells. Cells plated in triplicate. D.) Transfection of Mcf10aneoT (GRHL2 WT, O/E, K.O.) cells with $2 \mathrm{ug} / \mathrm{mL}$ poly(I:C) revealed heightened cell death in GRHL2 O/E cells compared with both WT and K.O. using PrestoBlue cell viability assay. Untreated assayed in duplicate, treated assayed in quadruplicate. E.) $m R N A$ expression levels (FPKM) of TRAIL (TNFSF10) in triplicate generated from an RNAseq on Mcf10aneoT (GRHL2 WT or K.O.) before and after poly(I:C) treatment.

\section{Chapter II: Conclusion}

ICAM-1 is an interferon stimulated gene whose expression was upregulated by GRHL2 basally, and upon IFN- $\gamma$ stimulation. This provoked the hypothesis that GRHL2 played a role in transducing or promoting IFN signaling and subsequent gene expression. qPCR analysis of HT1080 and Mcf10aneoT cell lines supported this hypothesis; although our sample size of ISGs was too small to be able to make broad generalization. We did find that GRHL2 suppresses the prolific "intrinsic interferon genes" expressed in early pluri- and multi- potent murine stem cells, although the application of this is questionable since murine and human embryonic stem 
cells are not completely equitable systems; the timing of GRHL2 expression varies during development between species. For whole transcriptome RNAseq, our approach of stimulating interferon pathways via two distinct mechanisms (IFN- $\gamma$, poly(I:C)) across GRHL2 WT, O/E, K.O. cell lines gave us much broader coverage and sample size to explore not only individual genes, but also pathways altered by GRHL2 expression, or absence, and their implications for Type I and Type II interferon responses.

RNAseq analysis of Mcf10aneoT +GRHL2 WT, O/E, K.O. cells revealed that GRHL2 does regulate the dsRNA response and IFN- $\gamma$ response. We focused on the dsRNA response, as GRHL2 WT and O/E cells exhibited robust induction of dsRNA response genes, while GRHL2 K.O. cells showed deficits, most strikingly characterized by inability to produce Type I and Type III interferon. Our RNAseq data also provides numerous hypothesis-generating pieces of data to inform future experiments aiming to discover the mechanism by which GRHL2 influences interferon signaling (Table 3). Intriguingly, GRHL2 WT cells express about 2-fold higher MDA5 (IFIH1) mRNA under basal conditions compared to GRHL2 K.O. cells. MDA5 is one of the core cytosolic dsRNA sensors, implicated in responding to longer dsRNA molecules upon which it multimerizes, forming microfilaments. Upon poly(I:C) stimulation, MDA5 expression dramatically increases $\sim 18$-fold with an induced expression $\sim 10$-fold higher than the induced expression level in GRHL2 K.O. cells. This suggests that GRHL2 K.O. cells, at baseline, are less prepared to mount an antiviral response, at least through this pathway. Concerning the MDA5 pathway, our RNAseq also revealed that a key MDA5 negative regulator, RIOK3, is induced strongly by poly(l:C) in GRHL2 K.O. cells ( $\sim \rightarrow \sim 25$ FPKM), while in GRHL2 WT cells it remains poly $(\mathrm{I}: \mathrm{C})$ non-responsive. RIOK3 functions as a Serine/threonine kinase that phosphorylates 
MDA5's c-terminal region. This disrupts the ability of MDA5 to multimerize with dsRNA.

Functionally, this leads to dramatic reduction in IFN- $\beta$ production, which is rescued in RIOK3 K.O. cells (Takashima, Oshiumi, Takaki, Matsumoto, \& Seya, 2015).

Introduced earlier, the interferon regulatory factor 3 (IRF3) protein represents a critical portal for transmission of dsRNA stimulation to nuclear signaling. IRF3 protein is regulated posttranslationally through multiple modes (J. Liu, Qian, \& Cao, 2016). Our RNAseq data suggests that GRHL2 would be a positive regulator of IRF3 stability by promoting expression of various IRF3 regulators. ISG15 is a ubiquitin-like molecule that can be attached to and stabilizes IRF3. This process, termed "ISGylation", is also mediated by HERC5, which acts as a ubiquitin ligase for ISG15 (Wong, Pung, Sze, \& Chin, 2006). ISG15 is roughly 2.5x more highly expressed basally in GRHL2 WT cells, and upon poly(l:C) treatment, its expression is also strongly favored in GRHL2 WT cells. HERC5, although essentially not expressed in either cell line prior to poly(l:C) stimulation, is induced roughly 7x in GRHL2 WT cells, while it is essentially not turned on in GRHL2 K.O. cells. Another piece of evidence implicating IRF3 as a prime suspect of GRHL2's IFN promotion is TICAM1. Also known as TRIF, TICAM1 is an adaptor molecule that transmits signal from toll-like receptors to other downstream proteins. TICAM1 is specific for TLR3, the dsRNA receptor, and is shown to be essential for subsequent production of IFN- $\beta$ (Oshiumi, Matsumoto, Funami, Akazawa, \& Seya, 2003). Our RNAseq data shows that TICAM1 is $>2 x$ more highly expressed in GRHL2 WT cells at basal conditions, and also retains slightly higher expression after poly(I:C) stimulation than GRHL2 K.O. cells. Conversely, CFLAR (or FLIP) is a negative regulator of type I IFN response. Loss of CFLAR has been associated with increased poly(I:C) induced apoptosis and increased NF-kB and IRF3 response genes. Interestingly, basal 
CFLAR levels were equal between GRHL2 WT and K.O. cells, but showed strong, though

variable, upregulation only in GRHL2 K.O. cells. CFLAR has pro-survival activities through its

binding to TRADD and FADD, thereby blocking association with Caspase 8 and dulling the

apoptotic response (Handa, Tupper, Jordan, \& Harlan, 2011). The strong upregulation of CFLAR

in GRHL2 K.O. cells, accompanied by mild deficiency in poly(l:C) mediated TRAIL (TNFSF10)

upregulation could provide an explanation for the observed survival advantage of GRHL2 K.O.

cells during transfection of poly(I:C).

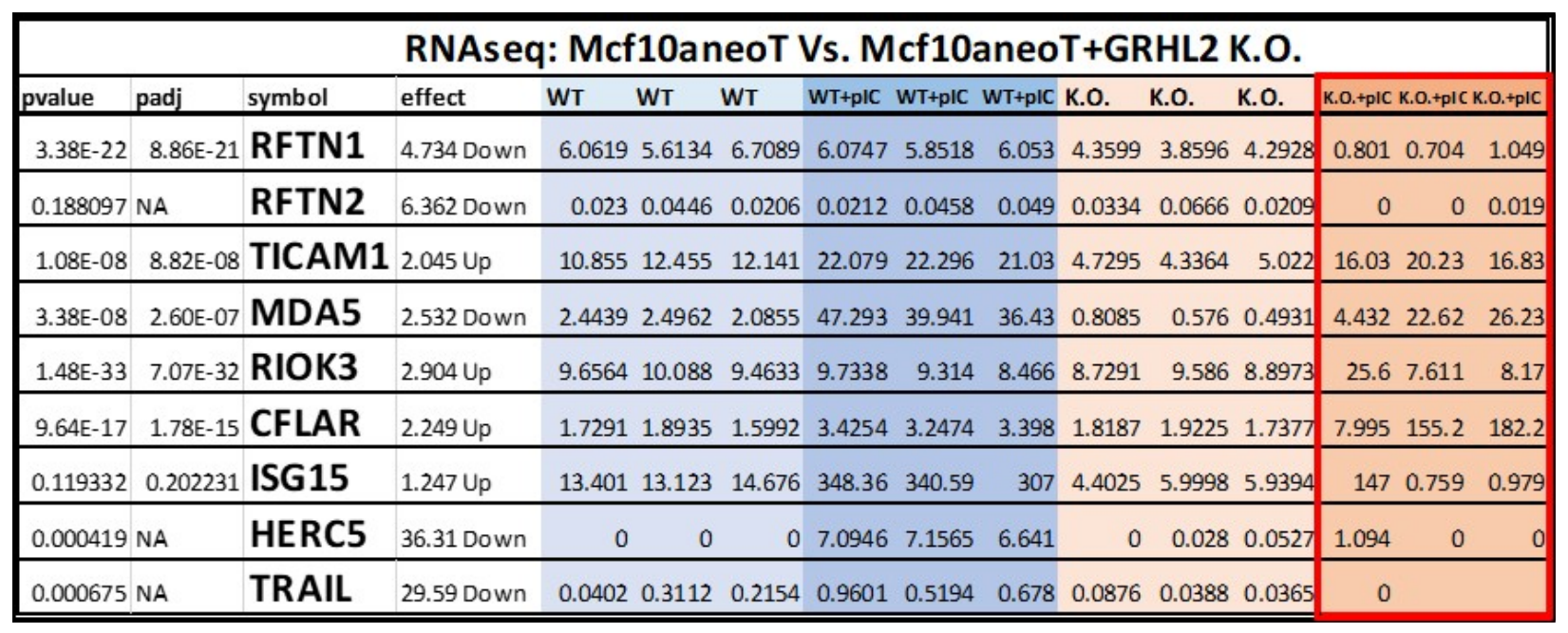

Table 3. Interferon related genes regulated by GRHL2. Raw RNAseq expression values in triplicate from Mcf10aneoT versus Mcf10aneoT +GRHL2 K.O. treated with $50 \mathrm{ug} / \mathrm{mL}$ poly(I:C) for 3hrs. RFTN1: Raftlin 1, RFTN2: Raftlin 2, TICAM1: toll-like receptor adaptor molecule 1, MDA5: interferon induced with helicase C domain 1, RIOK3: RIO Kinase 3, CFLAR: CASP8 and FADD like apoptosis regulator, ISG15: interferon stimulated gene 15, HERC5: HECT and RLD domain containing E3 ubiquitin protein ligase 5, TRAIL: TNF superfamily member 10. 
Production of Type I interferon is an important mediator of antiviral and antitumor immunity. Besides communicating a viral distress response to nearby cells, IFN- $\beta$ can cause cellcycle arrest, senescence and apoptosis in surrounding tumor cells (Snell et al., 2017). Apoptosis is mediated through TRAIL upregulation in melanoma and breast cancer, and Caspase- 8 activation in cervical carcinoma (Apelbaum et al., 2012; Bernardo et al., 2013; Chawla-Sarkar M., 2001). But this direct cytostatic and apoptotic effect must also be interpreted in light of the diverse impact that IFN-I has on the immune microenvironment: effecting dendritic cells, macrophages, B cells, CD4+ and CD8+ T cells (Snell et al., 2017) , as well as the timing and duration of its signaling. Although inflammatory in nature, chronic IFN-I signaling can also lead to upregulation of PD-L1 and immunosuppression: altering the phenotype and activity of cells of the innate and adaptive immune system (Snell et al., 2017). It is important that cells counterregulate IFN-I production; demonstrated by dramatic reduction in IFN-I expression after the initial acute phase, though many ISGs are still expressed, or are primed for expression in this context (Snell et al., 2017). We currently have no evidence to suggest that GRHL2 WT or O/E cells have an inability to turn off, or reduce IFN-I production after the initial acute phase following stimulation, though it would be interesting to determine if the kinetics of ISG expression were altered in GRHL2 WT versus O/E; are ISGs more rapidly produced initially, and IFN-I production slower to diminish after the acute phase in cells constitutively overexpressing GRHL2?

In future studies we would like to address this question within the context of a proposed role for GRHL2 in epigenetically priming interferon response genes. Intriguingly, GRHL2 binding sites are located in regulatory regions of various ISGs, and overlap with some 
interferon regulatory factor (IRFs) binding sites. The extent to which GRHL2 preferentially, nonrandomly locates to interferon related genes, above its distribution across the rest of the genome, would give us an indication of its potential as an epigenetic regulator of the interferon response, perhaps in a manner similar to its regulation of epithelial/mesenchymal genes (via KMT2C/D recruitment, p300 inhibition.) This appeared to be GRHL2's manner of regulating the ISG ICAM-1, as demonstrated by our CHIP-qPCR; KMT2C was not recruited to ICAM-1 promoter in the absence of a GRHL2 interaction (GRHL2 ED $\rightarrow$ AA cells) effectively silencing this gene.

GRHL2 is an epithelial master transcription factor, responsible for overseeing a wide range of lineage determining genes, however, inclusion of interferon stimulated genes, particularly in the presence of dsRNA or IFN- $\gamma$ was unforeseen. This raises important implications not only for cancer related properties and anti-tumor immunity, but also for viral infection of epithelial cells. Future experiments should address the infection and replication competence of virus in epithelial cells in the presence (WT) or absence (K.O.) of GRHL2. Our data showed that GRHL2 recruited KMT2C to the ISG ICAM-1 promoter in a GRHL2-dependent manner. Future experiments should explore if this mode of epigenetic regulation is conserved in promoter/enhancer regions of various other ISGs. This could be quantified by CHIP-seq of epithelial cells expressing endogenous GRHL2 before and after poly(I:C) treatment. Does GRHL2 bind these genes upon stimulation with poly(l:C)? A complementary approach would involve ATAC-seq of accessible chromatin regions before and after poly(l:C) in epithelial cells expressing endogenous GRHL2 or GRHL2 K.O. This would indicate the importance of GRHL2 in establishing open, transcriptionally permissive sites at ISGs upon stimulation with ligand. As suggested above, these experiments could also be conducted with GRHL2 O/E epithelial cells toaddress 
whether increasing levels, or enforced overexpression, of GRHL2 causes a sustained or noncounter-regulated interferon response, specifically implicating sustained GRHL2-mediated epigenetic control. This could have implications for the tumor microenvironment and immune cells, as chronic type I IFN production has been shown to be immunosuppressive (S. Lukhele, G. M. Boukhaled, \& D. G. Brooks, 2019).

To conclude, GRHL2 has emerged as an important regulator of immune sensitivity. The presence of GRHL2 protein maintains expression of immune sensitizing genes such as ICAM-1 through epigenetic interactions. Expanding GRHL2's well documented role as an epithelial master transcription factor, our data also brings a multitude of interferon stimulated genes into GRHL2's wheelhouse of regulated genes. This data further solidifies the notion that appropriate immunological relationships are a facet of the epithelial state, as mediated by the master transcription factor GRHL2. 


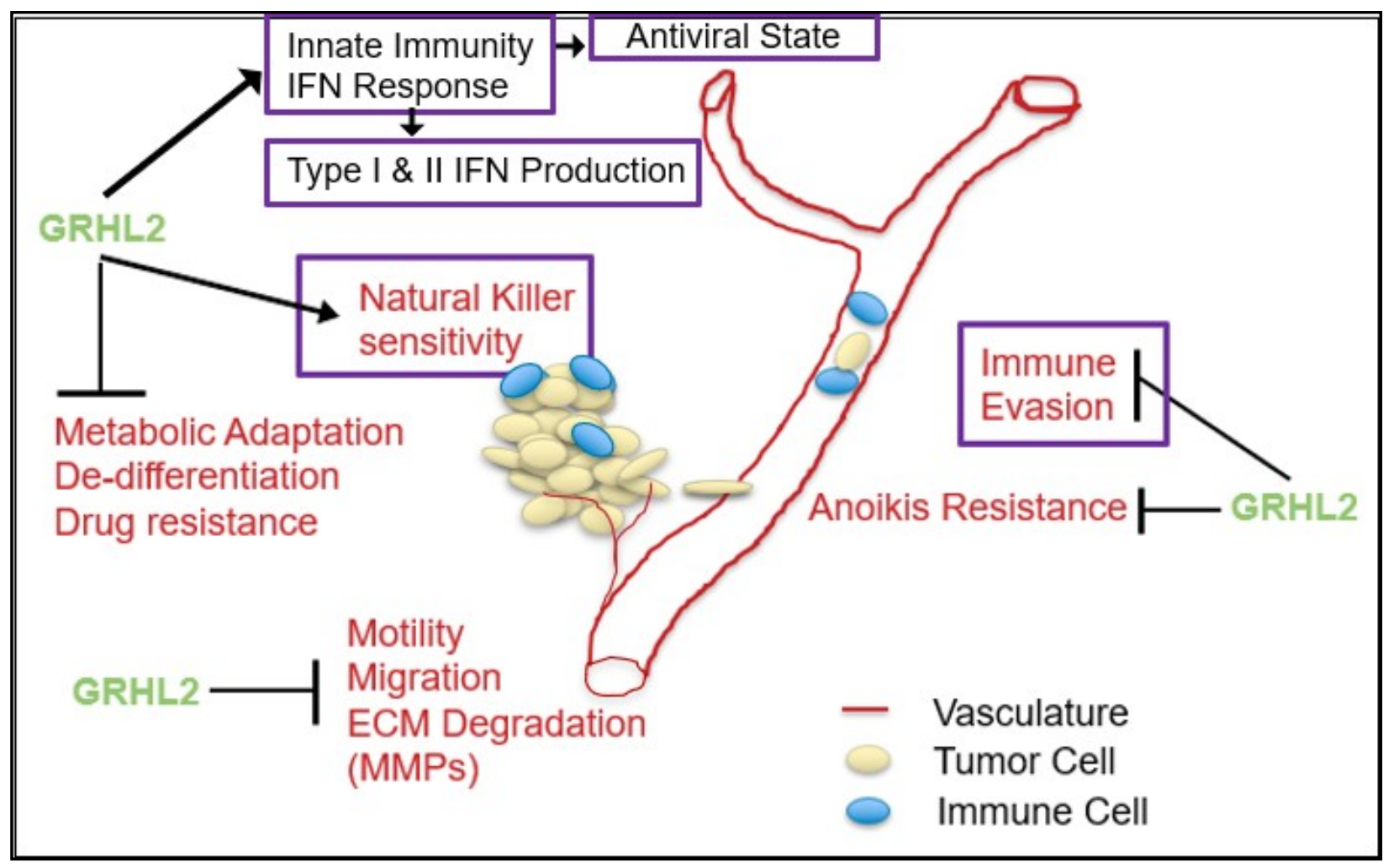

Diagram 3. The significance of GRHL2 in tumor biology. Novel proposed roles boxed in purple

\section{Materials and Methods}

\section{Cell lines}

MCF10a-derived, HMLE-derived and HT1080-derived cell lines were obtained and cultured as described previously. MCF10aneoT cells were induced to undergo EMT by treatment with $20 \mathrm{ng} / \mathrm{ml} \mathrm{TGF}-\beta$ for three days, and then outgrowth in the absence of TGF- $\beta$. Mesenchymal cell lines (MCF10aneoT+ TGF- $\beta$, abbreviated as NTM, the Mesenchymal Subpopulation derived from HMLE, abbreviated as MSP, HT1080 fibrosarcoma cells and BT549 triple negative breast cancer cells) were reverted to an epithelial phenotype by retroviral expression of GRHL2 in the 
vector pMXS-IRES-puro, as described previously, or by expression of mir200c (see below). NK92-MI cells (American Type Culture Collection) were cultured in Alpha Minimum Essential medium without ribonucleosides and deoxyribonucleosides but with $2 \mathrm{mM} \mathrm{L-glutamine}$ and 1.5 g/L sodium bicarbonate (Life Technologies) containing $0.2 \mathrm{mM}$ myo-inositol, $0.1 \mathrm{mM}$ 2mercaptoethanol, $0.02 \mathrm{mM}$ folic acid, $10 \%$ horse serum, $10 \%$ fetal bovine serum and $5 \mathrm{ng} / \mathrm{ml} \mathrm{IL-}$ 2 ( $R$ and D Systems). BT549 cells (grown in RPMI-1640+5\% fetal bovine serum $+10 \mu \mathrm{g} / \mathrm{ml}$ insulin+penicillin-streptomycin-glutamine) with inducible mir200c expression were constructed by subcloning the mir200c sequence into pTRIPZ, packaging lentiviruses, infecting BT549 cells and selecting for puromycin-resistance. The CRISPR/cas9 lentiviral constructs for knocking out human GRHL2 were kindly provided by Dr. B. Hogan, which were packaged in 293T cells and infected into target cell lines, as we described previously. GRHL2 knockout (>95\%) was verified by Western blotting total puromycin-resistant populations; subcloning of knockout cells proved unnecessary. The CRISPR/cas9 mediated knockouts of KMT2C and KMT2D were generated by subcloning guide sequences (For KMT2C: M3CrE3-F: caccgGCGATCTGTGTCTGAGGAAT M3CrE3-R: aaacATTCCTCAGACACAGATCGCC. For KMT2D: M4CrE3-F: caccgTCCCGCTGCCCGTGTAGACT

M4CrE3-R: aaacAGTCTACACGGGCAGCGGGAC) into the BsmBI sites of pLenti-Crispr-v2 or pLKO5.sgRNA.EFS.tRFP (Addgene). Lentiviruses were packaged and infected into MCF10a (subclone MCF10aP) cells as described, followed by sequential selection for puromycinresistance and flow-sorting for RFP. Indels that caused reading frame shifts were identified by generating PCR fragments from genomic DNA, which were subcloned into pTOPOII (Life Technologies) and sequenced. MCF7 and MCF7-EMT cell lines were kindly provided by Dr. 
Bruce White (University of Connecticut). HT1080+E1a cells were described previously. To generate target cells expressing nano-luciferase, cell lines were infected with the Nanoluciferase expressing lentiviral construct pLenti6.2-Nanoluc-ccdb (Addgene) and selected for blasticidin resistance $(10 \mu \mathrm{g} / \mathrm{ml})$.

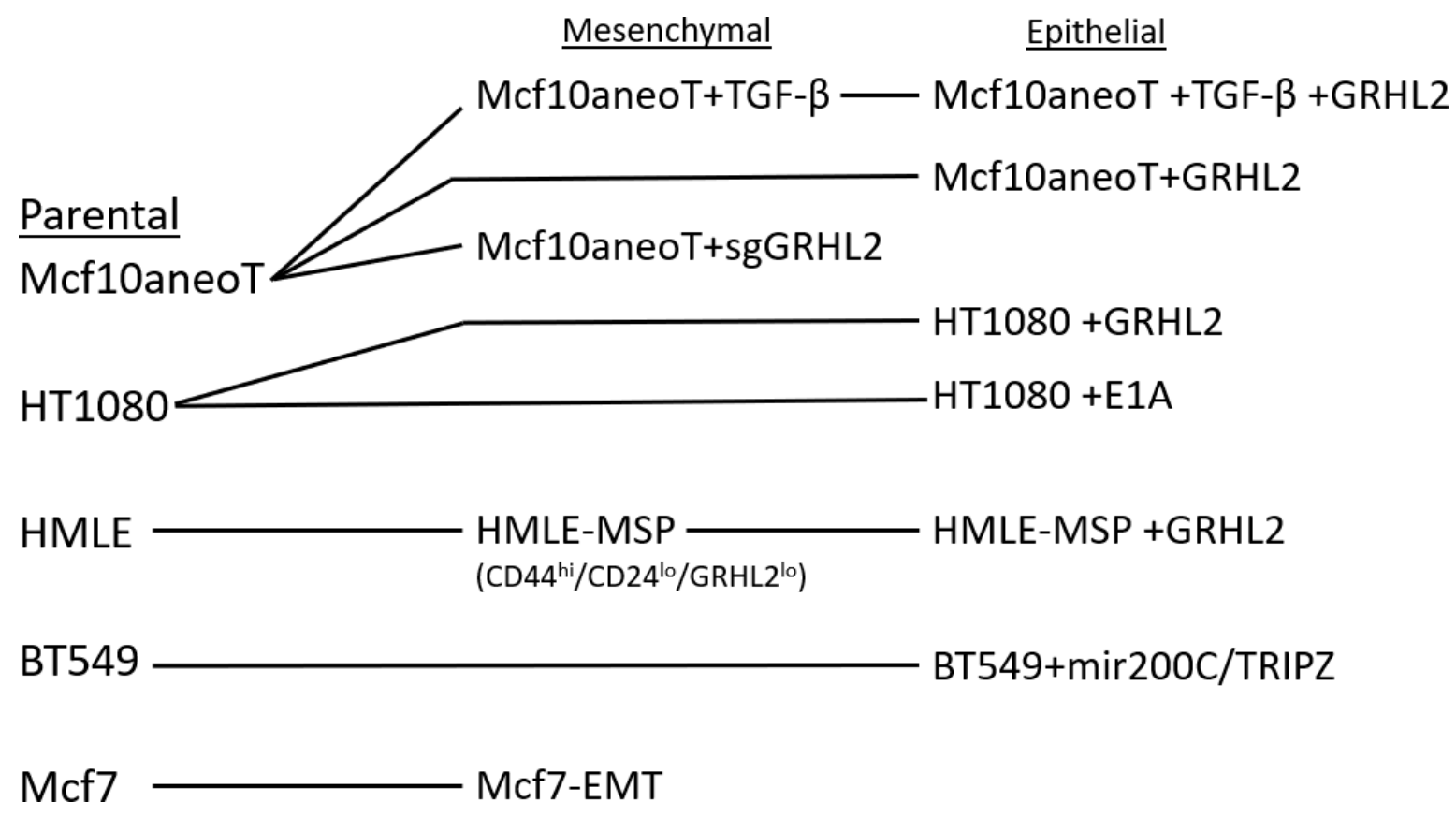

Western Blots

Cells were lysed in $1 x$ LDS Sample buffer containing 50mM DTT, then heated at 70C. Lysates were run on Novex $4-12 \%$ Bis-tris gels at $120 \mathrm{~V}$ for $2 \mathrm{hrs}$. After transfer, membranes were incubated with indicated antibodies, then developed using SuperSignal West Pico Chemiluminescent substrate.

NK cytotoxicity assays

Assay 1: Target cells were plated in 12-well format at $3.5 \times 10^{4}$ cells per well in triplicate. At least one day later, target cells were stained with $1 \mu \mathrm{M}$ CMFDA for $25 \mathrm{~min}$ in serum free medium, and washed 3x with complete medium. NK92-MI cells were added to wells in the 
appropriate target cell medium at 3:1 effector to target ratio. At the time points indicated, wells were gently washed $3 x$ with complete medium, and cells were lysed in $300 \mathrm{uL}$ of $3 \%$ SDS solution; 100uL of this sample was read in a black opaque 96-well plate (492 excitation/530 emission) in a BioTek Synergy H1 Hybrid Reader. Signal from (+)NK wells divided by (-) NK wells to calculate viability.

Assay 2: This assay was developed and validated for NK killing previously. Nano-luc expressing target cells (see above) were plated at $3.5 \times 10^{4}$ cells in triplicate in a 12 -well plate. The following day, NK92-Ml cells were added in target cell medium, and, at the indicated time points, $25 \mu \mathrm{L}$ aliquots of the culture supernatant were assayed using the Nano-Luc Assay System (Promega). To determine percentage of cells killed by NKs, a non-NK-treated well of target cells was lysed in $500 \mu \mathrm{L}$ of Cell culture lysis buffer (Promega) to measure total nano-luciferase activity.

FASL cytotoxicity was assayed by plating cells at $4.0 \times 10^{4}$ per well in triplicate in 12 -well plate.

The next day, Super-FASL (Enzo) was added to $50 \mathrm{ng} / \mathrm{mL}$. At the indicated timepoints, cells were washed $2 \mathrm{x}$ with $1 \mathrm{X}$ PBS and assayed for caspase activation using the Caspase-glo system (Promega).

To assay cells for granzyme B cytotoxicity, target cells plated at $4.0 \times 10^{4}$ cells per well in 12 -well plate. The following day, cells were washed in serum-free medium. Cells were permeabilized to allow granzyme B entry using streptolysin-O (Sigma) at concentrations that were preoptimized to produce low spontaneous death but promote granzyme B killing (typically, about $100 \mathrm{ng} / \mathrm{ml}$ ). Granzyme B, purified as described previously from YT cells, was added to 400 $\mathrm{ng} / \mathrm{mL}$ and wells were monitored for cell death. At the indicated time points, 0.1 volumes of 
Presto Blue Viability Reagent (Life Technologies) was added to wells and incubated $20 \mathrm{~min}$ at $37^{\circ}$ C. Aliquots $(150 \mu \mathrm{L})$ were assayed for fluorescence (560nm excitation/ 590nm emission) in opaque, black 96-well plates. Presto blue signal from wells treated with streptolysin-O alone were used to calculate viability.

\section{Autophagic Flux Assays}

HT1080 +/- GRHL2 cells were retrovirally infected with a tandem GFP/RFP-LC3 vector, then flow sorted to select for GFP(+) cells. Following selection, HT1080 +/-GRHL2 tfLC3 cells were plated onto coverslips in wells of a 6 -well plate at $\sim 50 \%$ confluence. $24 \mathrm{hrs}$ later cells were Serum Starved in HBSS for 2 hours, then either fixed or treated with Bafilomycin (100nM) for $2 \mathrm{hrs}$. Cells were then fixed in $2 \%$ formaldehyde for 10 min then permeabilized in $0.5 \%$ TX100 in PBS for $10 \mathrm{~min}$. The slides were then imaged using a Zeiss Axiovert 200M Fluorescence Microscope. Fields were then quantified for LC3 punctae using NIS Elements AR software in order to generate the autophagic flux for each cell line. Fields quantified: $n=4$ for all cell lines (except GRHL2+baf. $n=5$ ). Cells quantified MXS: $n=2507$, MXS+baf: n=5298, GRHL2: n=2658, GRHL2+Baf: $n=8294$

Autophagic Flux= [(Baf. Treated \# LC3 punctae) - (Untreated \# LC3 punctae $)]$ (pcDH1-tflc3.dna was a generous gift from Dr. Wang, Penn State)

\section{NK Conjugation Assay}

Target cells were plated at $3.5 \times 10^{4}$ cells/well in 12 well plate. $24 \mathrm{hrs}$ later, target cells were stained with $1 \mu \mathrm{M}$ CMPTX in Serum Free medium for 25min and NK92-MI cells were stained with $1 \mu \mathrm{M}$ CMFDA in serum Free medium for 25 minutes. Cells were washed $3 \mathrm{X}$, then stained NK92-MI cells were resuspended and added to stained target cells in 3:1 effector/target ratio in 
the presence of $50 \mu \mathrm{M}$ ZVAD-FMK. At time points, cells were gently washed $3 x$ with complete medium to remove unconjugated NK92-MI cells, then fixed in $2 \%$ paraformaldehyde for 10 min and washed $3 \times X$. The plates were then imaged at $10 x$ and $20 x$ magnification using an epifluorescence microscope (Zeiss Axiovert). GFP and Texas Red Channels were used to detect CMFDA and CMPTX stain, respectively. Images were then quantified for NK/target ratios using Image J software from the WVU Microscope Core Imaging Facility.

Anoikis Assay

Cells were plated at equal densities into low attachment cell culture plates in Mcf10a media $+0.5 \%$ methylcellulose. Cells were incubated at $37 \mathrm{C} / 5 \% \mathrm{CO}_{2}$. At time points, cells were resuspended and an aliquot was taken and mixed 1:1 with Caspase 3/7 glo reagent (Promega), incubated for 30', then read on a Wallac Envision Platereader to quantify anoikis.

$q R T-P C R$

Duplicate or triplicate total RNAs were prepared from cells in $60 \mathrm{~mm}$ dishes using the Qiagen RNeasy Plus Mini kit. RNA (2.5 $\mu \mathrm{g}$ ) were converted to single strand cDNA using the Superscript III kit (Life Technologies). One microliter of (3:1 diluted) cDNA was assayed in $20 \mathrm{uL}$ reactions using the PowerUp SYBR green master mix (Applied Biosystems) on an Applied Biosystems 7500 thermocycler. GAPDH was used as the internal control for normalization.

The following primer sets were used:

\begin{tabular}{|l|l|l|}
\hline gene & Primer $\mathrm{F}$ & Primer $\mathrm{R}$ \\
\hline DCN & agaagctctcctacatccgc & ccaagtgaagctccctcaga \\
\hline SRGN & attttcccaccttgacacca & ttttatggccatgggaatat \\
\hline ZEB1 & catacacctactcaactac & tttcatcctgatttccatt \\
\hline
\end{tabular}




\begin{tabular}{|l|l|l|}
\hline Col8A1 & catttaccgccgagctaacc & tgtctgcgggttgtagttct \\
\hline Snail & gaaaggccttcaactgcaaa & tgacatctgagtgggtctgg \\
\hline Twist & cgggagtccgcagtctta & gcttgagggtctgaatcttg \\
\hline $\begin{array}{l}\text { Rab25- } \\
\text { CDH1- } \\
2\end{array}$ & cctaaccaagcaccagacct & gctgttctgtctctgcttgg \\
\hline $\begin{array}{l}\text { ESRP1- } \\
2\end{array}$ & tttgaatccacgagcactgc & taagtccatcctcggttgca \\
\hline $\begin{array}{l}\text { MMP2- } \\
2\end{array}$ & aggaggagaaggctgtgttc & ctccagttaaaggcggcatc \\
\hline TP63-2 & atgcccagactcaatttagtga & ttctgcgcgtggtctgtgt \\
\hline OVOL2 & tgcaacgtctgcaataaagc & acgtgcaggtacaggtcctc \\
\hline EPCAM & tgcagggtctaaaagctggt & ccctatgcatctcacccatc \\
\hline ITGB6 & tgcgaccatcagtgaagaag & gacaacccgatgagaagaa \\
\hline CLDN4 & ccggccttatggtgatagtg & gcggagtaaggcttgtctgt \\
\hline IRF6 & gtgaatgccatcttcttccttct & cccaggccaaatctccttct \\
\hline $\begin{array}{l}\text { ICAM1- } \\
2\end{array}$ & atacaacagcatttggggcc & ccacttcccctctcatcagg \\
\hline Wnt5a & cccgatttagcagtgtcagc & tacacagtgccagtctcagg \\
\hline RAET1L & cttctgttcctgctgttcgg & cgggtgtgactgtcttgttg \\
\hline S100A4 & gagcaacttggacagcaaca & tcatttcttcctgggctgct \\
\hline $\begin{array}{l}\text { CD112- } \\
2\end{array}$ & ccatgtatgtgtgagctgcc & tttctctgggctcatgggtt \\
\hline $\begin{array}{l}\text { CD155- } \\
2\end{array}$ & tggggtgaaaatgtctgtgc & ggtttactccagcctccact \\
\hline GAPDH & tgcaccaccaactgcttagc & ggcatggactgtggtcatgag \\
\hline & & \\
\hline
\end{tabular}

Antibodies

\begin{tabular}{|l|l|l|l|}
\hline Antibody & Application & Company & Catalogue \#/Ref. \\
\hline E-Cadherin & Western Blot & BD Bioscience & 610181 \\
\hline WDR5 & Western Blot & Cell Signaling & D9E1I \\
\hline GRHL2 & CHIP/Western Blot & Sigma & AMab91226 \\
\hline KMT2C & CHIP & $\begin{array}{l}\text { J. Wysocka lab } \\
\text { (Stanford) }\end{array}$ & (Dorighi et al., 2017) \\
\hline FLAG & Western Blot & Sigma & F3165-1MG \\
\hline
\end{tabular}




\begin{tabular}{|l|l|l|l|}
\hline ICAM1 & Western Blot & Santa Cruz & Sc-8439 \\
\hline AKT (pan) & Western Blot & Cell Signaling & C67E7 \\
\hline & & & \\
\hline
\end{tabular}

Chromatin Immunoprecipitation (CHIP)

CHIP assays were performed as previously described, with the primers listed below.

\begin{tabular}{|l|l|l|}
\hline Gene & Forward primer & Reverse primer \\
\hline Rab25 & GCTGTCTCTGAAGGTCCTGT & AGAGGACGGAAGCTGAGAAC \\
\hline CDH1 (5') & CCCCTATCAGTTAGCACCGT & AGGTAGGGTAGAGGAGGCTC \\
\hline CDH1 (intron 2) & GGTTCAAAAGATCCCCTGCG & GTCCCCTTTCCTAAGCCACA \\
\hline CLDN4 & TGGATGGACGGGTTTAGAGG & AGGACTCAGAGGGGATCAGT \\
\hline OVOL2 & TGCCTTAAATCGCGAGTGAG & CAGGTAGCGAGCTTGTTGAC \\
\hline TP63 & GGGGTGGGTGGTAAGGTATT & TTGGAAAGAACACTGCCTGC \\
\hline ICAM1 & ATAAAGGATCACGCGCCCC & AGCCCCTCCTTCCCATAAAC \\
\hline
\end{tabular}

Bioinformatics and statistics

Retrieval and analysis of the expression levels and EMT scores using publicly available data for lung cancer were achieved as follows. Raw expression values (counts) and Z-scores were retrieved for LUAD TCGA (provisional, freeze as of June 20,2018) dataset using CBioPortal. EMT scores were retrieved from the. For normalization to the IFN- $\gamma$ transcript levels, raw expression data were used (only samples with a detectable (non-zero) counts). Pearson correlation coefficients and the corresponding statistical significance values were calculated for the IFN- $\gamma$ normalized sets of the expression data. For all samples spanning the EMT continuum, the high and low subsets were defined as top and bottom quartiles of the scores for each EMT 
signature. For each gene of interest, the subsets of samples with high and low mRNA expression (normalized for IFN- $\gamma$ transcript levels) were also defined as top and bottom quartiles of the corresponding expression range. Statistical significance for the co-occurrence between EMT subsets and expression subsets was calculated based on binominal distribution. For NK assays, qPCR, and conjugation assaysStudent's Two Tailed T test was used to compare significance

Poly(l:C) Viability Assay:

Mcf10aneoT/ +GRHL2/ +gr sg2 were plated at 20,000 cells per well 12 well plates. 48 hrs later, 2ug of Poly:IC was mixed with 500uL optimem, mixed, then 3uL Lipofectaime RNAimax was added, mixed. Incubated for $15 \mathrm{mins}$. Added to cells that had been washed $1 \mathrm{x}$ and refed with Optimem. Incubated 37C for $4 \mathrm{hrs}$, washed $2 \mathrm{x}$ with normal medium. Incubated $14 \mathrm{hrs}$ in normal medium, then 50uL of PrestoBlue reagent added to each well. Incubated until good color change was observed, then read in a black 96 well plate on a platereader using PrestoBlue setting.

RNAseq Experiments

Mcf10aneoT, Mcf10aneoT+GRHL2, Mcf10aneoT+gr sg2 cells were plated in triplicate at equal, subconfluent, densities. Cells were treated with $50 \mathrm{ug} / \mathrm{mL}$ of poly(l:C) for $3 \mathrm{hrs}$, or $400 \mathrm{U} / \mathrm{mL}$ recombinant IFN- $\gamma$ for $12 \mathrm{hrs}$. Cells were then lysed and processed to make RNA using the Qiagen RNeasy Plus Mini kit. RNA was shipped to Genewhiz, where reads were conducted, then raw data was shipped to Dr. James Denvir (Marshall) for formatting. Fold induction was calculated by averaging triplicate FPKM after poly $(\mathrm{I}: \mathrm{C})$ treatment and dividing by triplicate 
average before ply(I:C) treatment. Genes were then ranked by fold induction. For GSEA

analysis, GSEA formatted .txt files were created by aligning the absolute expression (FPKM) after poly(l:C) stimulation for the triplicate samples from each cell line. This dataset was manually cleansed of most genes that were not expressed or had zero reads across all the samples. The GSEA formatted .txt file was uploaded to Broad Institute GSEA software and was queried against a curated set of "interferon" gene sets downloaded from "msigdatabase.org". These gene sets were curated by downloading all gene sets from msigdatabase.org that were found associated with the search term "interferon". Enrichments with a False Discovery Rate FDR $<0.25$ and a normalized enrichment score NES of $>1.2$ were considered.

\section{Extracellular Matrix Switch Experiments}

Extracellular matrix was deposited and isolated according to the methods described by (Au Hellewell, Au - Rosini, \& Au - Adams, 2017). Generally, cells were plated in multi-well tissue culture treated plates until confluent. Cells were washed, then briefly lysed using Ammonium hydroxide with agitation. Cellular and medium milieu suspension was then aspirated and wells were washed numerous times. This method removes cells, but leaves matrix behind relatively intact. Mesenchymal or epithelial derivatives were then plated at subconfluent densities on the opposing matrix type. After attachment, these cells were then subjected to an NK assay as described above to characterize matrix effects on sensitivity to NK92mi cells.

\section{Serglycin Overexpression/ Conditioned Media Experiments}

The long isoform of Serglycin codes for a cytosolic, non-secreted form. 
>NM_001321053 (longest isoform, contains signal peptide):

atgat gcagaagcta

361 ctcaaatgca gtcggcttgt cctggctctt gccctcatcc tggttctgga atcctcagtt

421 caaggttatc ctacgcggag agccaggtac caatgggtgc gctgcaatcc agacagtaat

481 tctgcaaact gccttgaaga aaaaggacca atgttcgaac tacttccagg tgaatccaac

541 aagatcccc gtctgaggac tgaccttttt ccaaagacga gaatccagga cttgaatcgt

601 atcttcccac tttctgagga ctactctgga tcaggcttcg gctccggctc cggctctgga

661 tcaggatctg ggagtggctt cctaacggaa atggaacagg attaccaactagtagacgaa

721 agtgatgctt tccatgacaa ccttaggtct cttgacagga atctgccctc agacagccag

781 gacttgggtc aacatggatt agaagaggat tttatgttat aa

$457 \mathrm{bp}$

>NP_001307982.1 serglycin isoform 1 precursor [Homo sapiens]

MMQKLLKCSRLVLALALILVLESSVQGYPTRRARYQWVRCNPDSNSANCLEEKGPMFELLPGESNKIPRL RTDLFPKTRIQDLNRIFPLSEDYSGSGFGSGSGSGSGSGSGFLTEMEQDYQLVDESDAFHDNLRSLDRNL PSDSQDLGQHGLEEDFML

cDNA was generated from HMLE-MSP cells using First Strand Protocol. cDNA was PCR amplified using primer sets designed to amplify the Long " $\mathrm{L}$ " isoform.

Primer Sequences:

SRGN-L-F:atgatgcagaagctactcaa

SRGN-R: ttataacataaaatcctctt

PCR product was gel extracted and purified, then re-amplified with SRGN primers that

contained Notl restriction enzyme sites.

SRGN (w/ Notl sites) Primer sequences:

SRGN-L-F2: tattaaGCGGCCGCCCACC atgatgcagaagctactcaa

SRGN-R2: tattaaGCGGCCGC ttataacataaaatcctctt 
After digestion with Notl restriction enzyme, product was PCR purified, then ligated into the vector "pMXS-ires-blast-delta-stuffer". Ligation reaction was transformed into STBL3 competent E.coli strain. Colonies were selected, midi-prepped and sent for sequencing by Sequetech $^{\mathrm{TM}}$ (Mountain View, CA) to verify insert. Plasmids were packaged into GP2 cells and retrovirus was harvested. Cells were then infected with SRGN retroviral construct and assayed for NK killing as described above.

For Conditioned Media experiments, cell lines expressing high levels of SRGN were cultured and allowed to approach confluence. Media was harvested from cells, concentrated, and western blotted to verify presence of secreted SRGN. This conditioned media was then exchanged for non-SRGN rich media on sensitive cells, and NK assay was conducted to determine effects of SRGN conditioned media on sensitive cells.

GRHL2 Immunohistochemistry Breast Cancer Tissue Microarray

We thank the IU Simon Cancer Center at Indiana University School of Medicine for the use of the Tissue Procurement \& Distribution Core, which provided Tumor Microarray Service. IHC staining was conducted by Dr. Naira Margaryan at WVU. GRHL2 protein staining was scored as either $0,+1,+2$, or +3 . Statistical analysis was conducted using a Student's Two Tailed T test. https://cancer.iu.edu/research-trials/facilities/tissue/policies.php 


\section{References}

Abe, T., Marutani, Y., \& Shoji, I. (2019). Cytosolic DNA-sensing immune response and viral infection. Microbiology and immunology, 63(2), 51-64. doi:10.1111/1348-0421.12669

Afonina, I. S., Cullen, S. P., \& Martin, S. J. (2010). Cytotoxic and non-cytotoxic roles of the CTL/NK protease granzyme B. Immunological Reviews, 235(1), 105-116. doi:10.1111/j.01052896.2010.00908.x

Akalay, I., Janji, B., Hasmim, M., Noman, M. Z., Andre, F., De Cremoux, P., . . Chouaib, S. (2013). Epithelial-to-mesenchymal transition and autophagy induction in breast carcinoma promote escape from T-cell-mediated lysis. Cancer Res, 73(8), 2418-2427. doi:10.1158/0008-5472.CAN$12-2432$

Al Absi, A., Wurzer, H., Guerin, C., Hoffmann, C., Moreau, F., Mao, X., ... Thomas, C. (2018). Actin Cytoskeleton Remodeling Drives Breast Cancer Cell Escape from Natural Killer-Mediated Cytotoxicity. Cancer Res, 78(19), 5631-5643. doi:10.1158/0008-5472.CAN-18-0441

Alam, H., Tang, M., Maitituoheti, M., Dhar, S. S., Kumar, M., Han, C. Y., . . Lee, M. G. (2020). KMT2D Deficiency Impairs Super-Enhancers to Confer a Glycolytic Vulnerability in Lung Cancer. Cancer Cell, 37(4), 599-617.e597. doi:10.1016/j.ccell.2020.03.005

Apelbaum, A., Yarden, G., Warszawski, S., Harari, D., \& Schreiber, G. (2012). Type I Interferons Induce Apoptosis by Balancing cFLIP and Caspase-8 Independent of Death Ligands. Mol Cell Biol, 33. doi:10.1128/MCB.01430-12

Artis, D., \& Spits, H. (2015). The biology of innate lymphoid cells. Nature, 517(7534), 293-301. doi:10.1038/nature14189

Au - Hellewell, A. L., Au - Rosini, S., \& Au - Adams, J. C. (2017). A Rapid, Scalable Method for the Isolation, Functional Study, and Analysis of Cell-derived Extracellular Matrix. JoVE(119), e55051. doi:doi:10.3791/55051

Aue, A., Hinze, C., Walentin, K., Ruffert, J., Yurtdas, Y., Werth, M., . . Schmidt-Ott, K. M. (2015). A Grainyhead-Like 2/Ovo-Like 2 Pathway Regulates Renal Epithelial Barrier Function and Lumen Expansion. J Am Soc Nephrol, 26(11), 2704-2715. doi:10.1681/ASN.2014080759

Bernardo, A. R., Cosgaya, J. M., Aranda, A., \& Jiménez-Lara, A. M. (2013). Synergy between RA and TLR3 promotes type I IFN-dependent apoptosis through upregulation of TRAIL pathway in breast cancer cells. Cell Death \& Disease, 4(1), e479-e479. doi:10.1038/cddis.2013.5

Besch, R., Poeck, H., Hohenauer, T., Senft, D., Hacker, G., Berking, C., ... Hartmann, G. (2009). Proapoptotic signaling induced by RIG-I and MDA-5 results in type I interferon-independent apoptosis in human melanoma cells. J Clin Invest, 119(8), 2399-2411. doi:10.1172/JCl37155

Boglev, Y., Wilanowski, T., Caddy, J., Parekh, V., Auden, A., Darido, C., . . Jane, S. M. (2011). The unique and cooperative roles of the Grainy head-like transcription factors in epidermal development reflect unexpected target gene specificity. Dev Biol, 349(2), 512-522. doi:S0012-1606(10)01198$X$ [pii]

10.1016/j.ydbio.2010.11.011

Bouris, P., Manou, D., Sopaki-Valalaki, A., Kolokotroni, A., Moustakas, A., Kapoor, A., ... Theocharis, A. D. (2018). Serglycin promotes breast cancer cell aggressiveness: Induction of epithelial to mesenchymal transition, proteolytic activity and IL-8 signaling. Matrix Biology, 74, 35-51. doi:https://doi.org/10.1016/j.matbio.2018.05.011

Bray, S. J., Burke, B., Brown, N. H., \& Hirsh, J. (1989). Embryonic expression pattern of a family of Drosophila proteins that interact with a central nervous system regulatory element. Genes Dev, 3(8), 1130-1145. 
Callari, M., Musella, V., Di Buduo, E., Sensi, M., Miodini, P., Dugo, M., ... Daidone, M. G. (2014). Subtype-dependent prognostic relevance of an interferon-induced pathway metagene in nodenegative breast cancer. Mol Oncol, 8(7), 1278-1289. doi:10.1016/j.molonc.2014.04.010

Chawla-Sarkar M., L. D., Borden EC. (2001). Preferential induction of apoptosis by interferon (IFN)-beta compared with IFN-alpha2: correlation with TRAIL/Apo2L induction in melanoma cell lines. Clinical Cancer Research, 7, 1821-1831.

Chen, A. F., Liu, A. J., Krishnakumar, R., Freimer, J. W., DeVeale, B., \& Blelloch, R. (2018). GRHL2Dependent Enhancer Switching Maintains a Pluripotent Stem Cell Transcriptional Subnetwork after Exit from Naive Pluripotency. Cell Stem Cell, 23(2), 226-238 e224. doi:10.1016/j.stem.2018.06.005

Chen, D. S., \& Mellman, I. (2017). Elements of cancer immunity and the cancer-immune set point. Nature, 541(7637), 321-330. doi:10.1038/nature21349

Chiappetta, C., Mancini, M., Lessi, F., Aretini, P., De Gregorio, V., Puggioni, C., . . . Di Cristofano, C. (2017). Whole-exome analysis in osteosarcoma to identify a personalized therapy. Oncotarget, 8(46), 80416-80428. doi:10.18632/oncotarget.19010

Cieply, B., Riley, P. t., Pifer, P. M., Widmeyer, J., Addison, J. B., Ivanov, A. V., . . Frisch, S. M. (2012). Suppression of the Epithelial-Mesenchymal Transition by Grainyhead-like-2. Cancer Res, 72(9), 2440-2453. doi:0008-5472.CAN-11-4038 [pii]

\subsection{8/0008-5472.CAN-11-4038}

Culley, F. J., Johnson, M., Evans, J. H., Kumar, S., Crilly, R., Casasbuenas, J., . . Davis, D. M. (2009). Natural Killer Cell Signal Integration Balances Synapse Symmetry and Migration. PLOS Biology, 7(7), e1000159. doi:10.1371/journal.pbio.1000159

Diamond, M. S., Kinder, M., Matsushita, H., Mashayekhi, M., Dunn, G. P., Archambault, J. M., ... Schreiber, R. D. (2011). Type I interferon is selectively required by dendritic cells for immune rejection of tumors. Journal of Experimental Medicine, 208(10), 1989-2003. doi:10.1084/jem.20101158

Doherty, M. R., Cheon, H., Junk, D. J., Vinayak, S., Varadan, V., Telli, M. L., . . Jackson, M. W. (2017). Interferon-beta represses cancer stem cell properties in triple-negative breast cancer. Proc Natl Acad Sci U S A, 114(52), 13792-13797. doi:10.1073/pnas.1713728114

Dongre, A., Rashidian, M., Reinhardt, F., Bagnato, A., Keckesova, Z., Ploegh, H. L., \& Weinberg, R. A. (2017). Epithelial-to-Mesenchymal Transition Contributes to Immunosuppression in Breast Carcinomas. Cancer Res, 77(15), 3982-3989. doi:10.1158/0008-5472.CAN-16-3292

Dorighi, K. M., Swigut, T., Henriques, T., Bhanu, N. V., Scruggs, B. S., Nady, N., . . Wysocka, J. (2017). MII3 and Mll4 Facilitate Enhancer RNA Synthesis and Transcription from Promoters Independently of H3K4 Monomethylation. Mol Cell, 66(4), 568-576 e564. doi:10.1016/j.molcel.2017.04.018

Dudas, J., Ladanyi, A., Ingruber, J., Steinbichler, T. B., \& Riechelmann, H. (2020). Epithelial to Mesenchymal Transition: A Mechanism that Fuels Cancer Radio/Chemoresistance. Cells, 9(2). doi:10.3390/cells9020428

Dustin, M. L. (2014). The Immunological Synapse. Cancer Immunology Research, 2(11), 1023. doi:10.1158/2326-6066.CIR-14-0161

Dustin, M. L., Rothlein, R., Bhan, A. K., Dinarello, C. A., \& Springer, T. A. (2011). Induction by IL 1 and interferon- $\gamma$ : tissue distribution, biochemistry, and function of a natural adherence molecule (ICAM-1). J. Immunol. 1986. 137: 245-254. J Immunol, 186(9), 5024-5033.

Dynlacht, B. D., Attardi, L. D., Admon, A., Freeman, M., \& Tjian, R. (1989). Functional analysis of NTF-1, a developmentally regulated Drosophila transcription factor that binds neuronal cis elements. Genes Dev, 3(11), 1677-1688. 
Elboim, M., Gazit, R., Gur, C., Ghadially, H., Betser-Cohen, G., \& Mandelboim, O. (2010). Tumor immunoediting by NKp46. J Immunol, 184(10), 5637-5644. doi:10.4049/jimmunol.0901644

Ellis, M. J., Ding, L., Shen, D., Luo, J., Suman, V. J., Wallis, J. W., . . Mardis, E. R. (2012). Whole-genome analysis informs breast cancer response to aromatase inhibition. Nature, 486(7403), 353-360. doi:10.1038/nature11143

Fagan, R. J., \& Dingwall, A. K. (2019). COMPASS Ascending: Emerging clues regarding the roles of MLL3/KMT2C and MLL2/KMT2D proteins in cancer. Cancer Lett, 458, 56-65. doi:10.1016/j.canlet.2019.05.024

Fantini, D., Glaser, A. P., Rimar, K. J., Wang, Y., Schipma, M., Varghese, N., . . Meeks, J. J. (2018). A Carcinogen-induced mouse model recapitulates the molecular alterations of human muscle invasive bladder cancer. Oncogene, 37(14), 1911-1925. doi:10.1038/s41388-017-0099-6

Farris, J. C., Pifer, P. M., Zheng, L., Gottlieb, E., Denvir, J., \& Frisch, S. M. (2016). Grainyhead-like 2 Reverses the Metabolic Changes Induced by the Oncogenic Epithelial-mesenchymal Transition: Effects on Anoikis. Mol Cancer Res. doi:10.1158/1541-7786.MCR-16-0050

Frisch, S. M. (1994). E1a induces the expression of epithelial characteristics. J Cell Biol, 127(4), 10851096.

Frisch, S. M. (1997). The epithelial cell default-phenotype hypothesis and its implications for cancer. Bioessays, 19(8), 705-709. doi:10.1002/bies.950190811

Frisch, S. M. (2001). Tumor suppression activity of adenovirus E1a protein: anoikis and the epithelial phenotype. Adv Cancer Res, 80, 39-49.

Frisch, S. M., Farris, J. C., \& Pifer, P. M. (2017). Roles of Grainyhead-like transcription factors in cancer. Oncogene, 36(44), 6067-6073. doi:10.1038/onc.2017.178

Fu, S., Wei, J., Wang, G., Wang, B., Wang, Y., Lai, X., \& Huang, H. (2015). The key role of PML in IFN- $\alpha$ induced cellular senescence of human mesenchymal stromal cells. Int J Oncol, 46(1), 351-359. doi:10.3892/ijo.2014.2738

Gala, K., Li, Q., Sinha, A., Razavi, P., Dorso, M., Sanchez-Vega, F., . . Chandarlapaty, S. (2018). KMT2C mediates the estrogen dependence of breast cancer through regulation of ER $\alpha$ enhancer function. Oncogene, 37(34), 4692-4710. doi:10.1038/s41388-018-0273-5

Gao, X., Bali, A. S., Randell, S. H., \& Hogan, B. L. (2015). GRHL2 coordinates regeneration of a polarized mucociliary epithelium from basal stem cells. J Cell Biol, 211(3), 669-682. doi:10.1083/jcb.201506014

García-Valtanen, P., Martínez-López, A., López-Muñoz, A., Bello-Perez, M., Medina-Gali, R. M., OrtegaVillaizán, M. d. M., . . Coll, J. (2017). Zebra Fish Lacking Adaptive Immunity Acquire an Antiviral Alert State Characterized by Upregulated Gene Expression of Apoptosis, Multigene Families, and Interferon-Related Genes. Frontiers in Immunology, 8(121). doi:10.3389/fimmu.2017.00121

Gibbons, D. L., \& Creighton, C. J. (2018). Pan-cancer survey of epithelial-mesenchymal transition markers across the Cancer Genome Atlas. Dev Dyn, 247(3), 555-564. doi:10.1002/dvdy.24485

Gorbunova V, Hine C, \& X, T. (2012). Cancer resistance in the blind mole rat is mediated by concerted necrotic cell death mechanism. Proc Natl Acad Sci U S A, 109, 19392-19396. doi:10.1073.pnas

Handa, P., Tupper, J. C., Jordan, K. C., \& Harlan, J. M. (2011). FLIP (Flice-like inhibitory protein) suppresses cytoplasmic double-stranded-RNA-induced apoptosis and NF-KB and IRF3-mediated signaling. Cell Commun Signal, 9, 16. doi:10.1186/1478-811X-9-16

He, J., Feng, C., Zhu, H., Wu, S., Jin, P., \& Xu, T. (2020). Grainyhead-like 2 as a double-edged sword in development and cancer. American journal of translational research, 12(2), 310-331.

Hersey, P., Edwards, A., Honeyman, M., \& McCarthy, W. H. (1979). Low natural-killer-cell activity in familial melanoma patients and their relatives. British journal of cancer, 40(1), 113-122. doi:10.1038/bjc.1979.147 
Howe, E. N., Cochrane, D. R., \& Richer, J. K. Targets of miR-200c mediate suppression of cell motility and anoikis resistance. Breast Cancer Res, 13(2), R45. doi:bcr2867 [pii]

\section{$10.1186 /$ bcr2867}

Iguchi-Manaka, A., Kai, H., Yamashita, Y., Shibata, K., Tahara-Hanaoka, S., Honda, S.-i., ... Shibuya, A. (2008). Accelerated tumor growth in mice deficient in DNAM-1 receptor. The Journal of experimental medicine, 205(13), 2959-2964. doi:10.1084/jem.20081611

Imai, D., Yoshizumi, T., Okano, S., Itoh, S., Ikegami, T., Harada, N., . . . Maehara, Y. (2019). IFN- $\gamma$ Promotes Epithelial-Mesenchymal Transition and the Expression of PD-L1 in Pancreatic Cancer. Journal of Surgical Research, 240, 115-123. doi:https://doi.org/10.1016/j.jss.2019.02.038

Imai, K., Matsuyama, S., Miyake, S., Suga, K., \& Nakachi, K. (2000). Natural cytotoxic activity of peripheral-blood lymphocytes and cancer incidence: an 11-year follow-up study of a general population. The Lancet, 356(9244), 1795-1799. doi:https://doi.org/10.1016/S01406736(00)03231-1

Jacobs, J., Atkins, M., Davie, K., Imrichova, H., Romanelli, L., Christiaens, V., . . Aerts, S. (2018). The transcription factor Grainy head primes epithelial enhancers for spatiotemporal activation by displacing nucleosomes. Nat Genet, 50(7), 1011-1020. doi:10.1038/s41588-018-0140-x

Jeong, J.-U., Uong, T. N. T., Chung, W.-K., Nam, T.-K., Ahn, S.-J., Song, J.-Y., . . Yoon, M. S. (2018). Effect of irradiation-induced intercellular adhesion molecule-1 expression on natural killer cellmediated cytotoxicity toward human cancer cells. Cytotherapy, 20(5), 715-727. doi:10.1016/j.jcyt.2018.01.010

Jolly, M. K., Somarelli, J. A., Sheth, M., Biddle, A., Tripathi, S. C., Armstrong, A. J., . . L Levine, H. (2019). Hybrid epithelial/mesenchymal phenotypes promote metastasis and therapy resistance across carcinomas. Pharmacol Ther, 194, 161-184. doi:10.1016/j.pharmthera.2018.09.007

Jolly, M. K., Tripathi, S. C., Jia, D., Mooney, S. M., Celiktas, M., Hanash, S. M., . . Levine, H. (2016). Stability of the hybrid epithelial/mesenchymal phenotype. Oncotarget, 7(19), 27067-27084. doi:10.18632/oncotarget.8166

Jozwik, K. M., Chernukhin, I., Serandour, A. A., Nagarajan, S., \& Carroll, J. S. (2016). FOXA1 Directs H3K4 Monomethylation at Enhancers via Recruitment of the Methyltransferase MLL3. Cell Rep, 17(10), 2715-2723. doi:10.1016/j.celrep.2016.11.028

Katlinski, K. V., Gui, J., Katlinskaya, Y. V., Ortiz, A., Chakraborty, R., Bhattacharya, S., . . Fuchs, S. Y. (2017). Inactivation of Interferon Receptor Promotes the Establishment of Immune Privileged Tumor Microenvironment. Cancer Cell, 31(2), 194-207. doi:10.1016/j.ccell.2017.01.004

Klingemann, H., Boissel, L., \& Toneguzzo, F. (2016). Natural Killer Cells for Immunotherapy - Advantages of the NK-92 Cell Line over Blood NK Cells. Front Immunol, 7, 91. doi:10.3389/fimmu.2016.00091

Korpetinou, A., Skandalis, S., Labropoulou, V., Smirlaki, G., Noulas, A., Karamanos, N., \& THEOCHARIS, A. (2014). Serglycin: At the Crossroad of Inflammation and Malignancy. Frontiers in Oncology, 3(327). doi:10.3389/fonc.2013.00327

Kotarba, G., Taracha-Wisniewska, A., \& Wilanowski, T. (2020). Grainyhead-like transcription factors in cancer - Focus on recent developments. Experimental Biology and Medicine, 245(5), 402-410. doi:10.1177/1535370220903009

Krebs, A. M., Mitschke, J., Lasierra Losada, M., Schmalhofer, O., Boerries, M., Busch, H., . . Brabletz, T. (2017). The EMT-activator Zeb1 is a key factor for cell plasticity and promotes metastasis in pancreatic cancer. Nature Cell Biology, 19(5), 518-529. doi:10.1038/ncb3513

Lafont, M., Vergnes, A., Vidal-Dupiol, J., de Lorgeril, J., Gueguen, Y., Haffner, P., . . Montagnani, C. (2020). A Sustained Immune Response Supports Long-Term Antiviral Immune Priming in the Pacific Oyster, \&lt;span class=\&quot;named-content genus-species\&quot; id=\&quot;named- 
content-1\&quot;\&gt;Crassostrea gigas\&lt;/span\&gt. mBio, 11(2), e02777-02719.

doi:10.1128/mBio.02777-19

Lee, J., Kim, D. H., Lee, S., Yang, Q. H., Lee, D. K., Lee, S. K., . . Lee, J. W. (2009). A tumor suppressive coactivator complex of p53 containing ASC-2 and histone H3-lysine-4 methyltransferase MLL3 or its paralogue MLL4. Proc Natl Acad Sci U S A, 106(21), 8513-8518. doi:10.1073/pnas.0902873106

Lee, M., Kim, D. W., Khalmuratova, R., Shin, S.-H., Kim, Y.-M., Han, D. H., . . Shin, H.-W. (2019). The IFN$\gamma-p 38$, ERK kinase axis exacerbates neutrophilic chronic rhinosinusitis by inducing the epithelialto-mesenchymal transition. Mucosal Immunology, 12(3), 601-611. doi:10.1038/s41385-0190149-1

Li, F., Tiede, B., Massagué, J., \& Kang, Y. (2007). Beyond tumorigenesis: cancer stem cells in metastasis. Cell Res, 17(1), 3-14. doi:10.1038/sj.cr.7310118

Li, Q., Tang, L., Roberts, P. C., Kraniak, J. M., Fridman, A. L., Kulaeva, O. I., . . Tainsky, M. A. (2008). Interferon Regulatory Factors IRF5 and IRF7 Inhibit Growth and Induce Senescence in Immortal Li-Fraumeni Fibroblasts. Molecular Cancer Research, 6(5), 770. doi:10.1158/1541-7786.MCR-070114

Lieberman, J. (2010). Granzyme A activates another way to die. Immunological Reviews, 235(1), 93-104. doi:10.1111/j.0105-2896.2010.00902.x

Liu, J., Qian, C., \& Cao, X. (2016). Post-Translational Modification Control of Innate Immunity. Immunity, 45(1), 15-30. doi:10.1016/j.immuni.2016.06.020

Liu, L., Kimball, S., Liu, H., Holowatyj, A., \& Yang, Z. Q. (2015). Genetic alterations of histone lysine methyltransferases and their significance in breast cancer. Oncotarget, 6(4), 2466-2482. doi:10.18632/oncotarget.2967

Lopez-Soto, A., Gonzalez, S., Smyth, M. J., \& Galluzzi, L. (2017). Control of Metastasis by NK Cells. Cancer Cell, 32(2), 135-154. doi:10.1016/j.ccell.2017.06.009

Lopez-Soto, A., Huergo-Zapico, L., Galvan, J. A., Rodrigo, L., de Herreros, A. G., Astudillo, A., \& Gonzalez, S. (2013). Epithelial-mesenchymal transition induces an antitumor immune response mediated by NKG2D receptor. J Immunol, 190(8), 4408-4419. doi:10.4049/jimmunol.1202950

Lukhele, S., Boukhaled, G. M., \& Brooks, D. G. (2019). Type I interferon signaling, regulation and gene stimulation in chronic virus infection. Seminars in Immunology, 43, 101277. doi:https://doi.org/10.1016/j.smim.2019.05.001

Lukhele, S., Boukhaled, G. M., \& Brooks, D. G. (2019). Type I interferon signaling, regulation and gene stimulation in chronic virus infection. Semin Immunol, 43, 101277. doi:10.1016/j.smim.2019.05.001

Lv, N., Gao, Y., Guan, H., Wu, D., Ding, S., Teng, W., \& Shan, Z. (2015). Inflammatory mediators, tumor necrosis factor- $\alpha$ and interferon- $\gamma$, induce EMT in human PTC cell lines. Oncol Lett, 10(4), 25912597. doi:10.3892/ol.2015.3518

MacFawn, I., Wilson, H., Selth, L. A., Leighton, I., Serebriiskii, I., Bleackley, R. C., . . Frisch, S. M. (2019). Grainyhead-like-2 confers NK-sensitivity through interactions with epigenetic modifiers. Mol Immunol, 105, 137-149. doi:10.1016/j.molimm.2018.11.006

Malaisé, M., Rovira, J., Renner, P., Eggenhofer, E., Sabet-Baktach, S.-R. M., Lantow, M., . . Kroemer, A. (2014). KLRG1(+) NK Cells Protect T-bet-Deficient Mice from Pulmonary Metastatic Colorectal Carcinoma. Journal of immunology (Baltimore, Md. : 1950), 192. doi:10.4049/jimmunol.1300876

Mancini, M., \& Vidal, S. M. (2020). Mechanisms of Natural Killer Cell Evasion Through Viral Adaptation. Annual Review of Immunology, 38(1), 511-539. doi:10.1146/annurev-immunol-082619-124440

Manou, D., Karamanos, N. K., \& Theocharis, A. D. (2020). Tumorigenic functions of serglycin: Regulatory roles in epithelial to mesenchymal transition and oncogenic signaling. Semin Cancer Biol, 62, 108-115. doi:10.1016/j.semcancer.2019.07.004 
Manso, L., Mourón, S., Tress, M., Gómez-López, G., Morente, M., Ciruelos, E., . . Q Quintela-Fandino, M. (2016). Analysis of Paired Primary-Metastatic Hormone-Receptor Positive Breast Tumors (HRPBC) Uncovers Potential Novel Drivers of Hormonal Resistance. PLoS One, 11(5), e0155840. doi:10.1371/journal.pone.0155840

Massagué, J., \& Obenauf, A. C. (2016). Metastatic colonization by circulating tumour cells. Nature, 529(7586), 298-306. doi:10.1038/nature17038

Messai, Y., Noman, M. Z., Hasmim, M., Janji, B., Tittarelli, A., Boutet, M., . . Chouaib, S. (2014). ITPR1 protects renal cancer cells against natural killer cells by inducing autophagy. Cancer Res, 74(23), 6820-6832. doi:10.1158/0008-5472.CAN-14-0303

Morin, R. D., Mendez-Lago, M., Mungall, A. J., Goya, R., Mungall, K. L., Corbett, R. D., ... Marra, M. A. (2011). Frequent mutation of histone-modifying genes in non-Hodgkin lymphoma. Nature, 476(7360), 298-303. doi:10.1038/nature10351

Morvan, M. G., \& Lanier, L. L. (2016). NK cells and cancer: you can teach innate cells new tricks. Nature Reviews Cancer, 16(1), 7-19. doi:10.1038/nrc.2015.5

Morvan, M. G., \& Lanier, L. L. (2016). NK cells and cancer: you can teach innate cells new tricks. Nat Rev Cancer, 16(1), 7-19. doi:10.1038/nrc.2015.5

Nagy, A., Lanczky, A., Menyhart, O., \& Gyorffy, B. (2018). Author Correction: Validation of miRNA prognostic power in hepatocellular carcinoma using expression data of independent datasets. Sci Rep, 8(1), 11515. doi:10.1038/s41598-018-29514-3

Nieto, M. A., Huang, Ruby Y.-J., Jackson, Rebecca A., \& Thiery, Jean P. (2016). EMT: 2016. Cell, 166(1), 21-45. doi:10.1016/j.cell.2016.06.028

Noman, M. Z., Janji, B., Abdou, A., Hasmim, M., Terry, S., Tan, T. Z., ... Chouaib, S. (2017). The immune checkpoint ligand PD-L1 is upregulated in EMT-activated human breast cancer cells by a mechanism involving ZEB-1 and miR-200. Oncoimmunology, 6(1), e1263412. doi:10.1080/2162402X.2016.1263412

Novakova, Z., Hubackova, S., Kosar, M., Janderova-Rossmeislova, L., Dobrovolna, J., Vasicova, P., . . . Hodny, Z. (2010). Cytokine expression and signaling in drug-induced cellular senescence. Oncogene, 29(2), 273-284. doi:10.1038/onc.2009.318

O'Sullivan, T., Saddawi-Konefka, R., Vermi, W., Koebel, C. M., Arthur, C., White, J. M., . . Bui, J. D. (2012). Cancer immunoediting by the innate immune system in the absence of adaptive immunity. The Journal of experimental medicine, 209(10), 1869-1882. doi:10.1084/jem.20112738

Oshiumi, H., Matsumoto, M., Funami, K., Akazawa, T., \& Seya, T. (2003). TICAM-1, an adaptor molecule that participates in Toll-like receptor 3-mediated interferon-beta induction. Nat Immunol, 4(2), 161-167. doi:10.1038/ni886

Osman, M., Burshtyn, D., \& Kane, K. (2007). Activating Ly-49 Receptors Regulate LFA-1-Mediated Adhesion by NK Cells. Journal of immunology (Baltimore, Md. : 1950), 178, 1261-1267. doi:10.4049/jimmunol.178.3.1261

Pan, X., Zhang, R., Xie, C., Gan, M., Yao, S., Yao, Y., . . Yu, B. (2017). GRHL2 suppresses tumor metastasis via regulation of transcriptional activity of RhoG in non-small cell lung cancer. American journal of translational research, 9(9), 4217-4226.

Parameswaran, R., Ramakrishnan, P., Moreton, S. A., Xia, Z., Hou, Y., Lee, D. A., ... Wald, D. N. (2016). Repression of GSK3 restores NK cell cytotoxicity in AML patients. Nat Commun, 7, 11154. doi:10.1038/ncomms11154

Pastushenko, I., \& Blanpain, C. (2019). EMT Transition States during Tumor Progression and Metastasis. Trends Cell Biol, 29(3), 212-226. doi:10.1016/j.tcb.2018.12.001

Pearson, G. W. (2019). Control of Invasion by Epithelial-to-Mesenchymal Transition Programs during Metastasis. J Clin Med, 8(5). doi:10.3390/jcm8050646 
Pedersen, L., Idorn, M., Olofsson, Gitte H., Lauenborg, B., Nookaew, I., Hansen, Rasmus H., ... Hojman, P. (2016). Voluntary Running Suppresses Tumor Growth through Epinephrine- and IL-6Dependent NK Cell Mobilization and Redistribution. Cell Metabolism, 23(3), 554-562. doi:10.1016/j.cmet.2016.01.011

Pifer, P. M., Farris, J. C., Thomas, A. L., Stoilov, P., Denvir, J., Smith, D. M., \& Frisch, S. M. (2016). Grainyhead-like 2 inhibits the coactivator p300, suppressing tubulogenesis and the epithelialmesenchymal transition. Mol Biol Cell, 27(15), 2479-2492. doi:10.1091/mbc.E16-04-0249

Poli, A., Michel, T., Thérésine, M., Andrès, E., Hentges, F., \& Zimmer, J. (2009). CD56bright natural killer (NK) cells: an important NK cell subset. Immunology, 126(4), 458-465. doi:10.1111/j.13652567.2008.03027.x

Quan, Y., Xu, M., Cui, P., Ye, M., Zhuang, B., \& Min, Z. (2015). Grainyhead-like 2 Promotes Tumor Growth and is Associated with Poor Prognosis in Colorectal Cancer. Journal of Cancer, 6(4), 342-350. doi:10.7150/jca.10969

Raja, S. M., Metkar, S. S., Honing, S., Wang, B., Russin, W.A., Pipalia, N.H., Menaa, C., Beling, M., Cao, X., Dressel, R., Froelich, C.J. (2005). A novel mechanism for protein delivery: Granzyme B undergoes electrostatic exchange from serglycin to target cells. Journal of Biological Chemistry, 280, 2075220761. doi:https://doi.org/10.1074/jbc.M501181200

Raja, S. M., Wang, B., Dantuluri, M., Desai, U. R., Demeler, B., Spiegel, K., . . Froelich, C. J. (2002). Cytotoxic cell granule-mediated apoptosis. Characterization of the macromolecular complex of granzyme B with serglycin. J Biol Chem, 277(51), 49523-49530. doi:10.1074/jbc.M209607200

Ray, H. J., \& Niswander, L. A. (2016). Grainyhead-like 2 downstream targets act to suppress epithelial-tomesenchymal transition during neural tube closure. Development, 143(7), 1192-1204. doi:10.1242/dev.129825

Reese, R. M., Harrison, M. M., \& Alarid, E. T. (2019). Grainyhead-like Protein 2: The Emerging Role in Hormone-Dependent Cancers and Epigenetics. Endocrinology, 160(5), 1275-1288. doi:10.1210/en.2019-00213

Ribatti, D., Tamma, R., \& Annese, T. (2020). Epithelial-Mesenchymal Transition in Cancer: A Historical Overview. Translational Oncology, 13(6), 100773. doi:https://doi.org/10.1016/j.tranon.2020.100773

Rogers, T., Christenson, J. L., Greene, L. I., O”Neill, K. I., Williams, M. M., Gordon, M. A., . . Richer, J. K. (2018). Reversal of Triple-negative breast cancer EMT by mir-200c decreases tryptophan catabolism and a program of immune-suppression. Mol Cancer Res, in press.

Rossignol, A., Bonnaudet, V., Clemenceau, B., Vie, H., \& Bretaudeau, L. (2017). A high-performance, nonradioactive potency assay for measuring cytotoxicity: A full substitute of the chromium-release assay targeting the regulatory-compliance objective. MAbs, 9(3), 521-535. doi:10.1080/19420862.2017.1286435

Sathe, P., Delconte, R. B., Souza-Fonseca-Guimaraes, F., Seillet, C., Chopin, M., Vandenberg, C. J., ... . Huntington, N. D. (2014). Innate immunodeficiency following genetic ablation of Mcl1 in natural killer cells. Nature Communications, 5(1), 4539. doi:10.1038/ncomms5539

Schneider, W. M., Chevillotte, M. D., \& Rice, C. M. (2014). Interferon-Stimulated Genes: A Complex Web of Host Defenses. Annual Review of Immunology, 32(1), 513-545. doi:10.1146/annurevimmunol-032713-120231

Shen, M., Xu, Z., Xu, W., Jiang, K., Zhang, F., Ding, Q., \& Chen, Y. (2019). Inhibition of ATM reverses EMT and decreases metastatic potential of cisplatin-resistant lung cancer cells through JAK/STAT3/PD-L1 pathway. J Exp Clin Cancer Res, 38(1), 149. doi:10.1186/s13046-019-1161-8

Smith, B. N., \& Bhowmick, N. A. (2016). Role of EMT in Metastasis and Therapy Resistance. J Clin Med, 5(2). doi:10.3390/jcm5020017 
Snell, L. M., McGaha, T. L., \& Brooks, D. G. (2017). Type I Interferon in Chronic Virus Infection and Cancer. Trends in Immunology, 38(8), 542-557. doi:10.1016/j.it.2017.05.005

Soares, E., \& Zhou, H. (2018). Master regulatory role of p63 in epidermal development and disease. Cellular and Molecular Life Sciences, 75(7), 1179-1190. doi:10.1007/s00018-017-2701-z

Song, N., Bai, M., Che, X., Li, Z., Jing, W., Li, C., . . Liu, Y. (2020). PD-L1 upregulation accompanied with epithelial-mesenchymal transition attenuates sensitivity to ATR inhibition in $\mathrm{p} 53$ mutant pancreatic cancer cells. Med Oncol, 37(5), 47. doi:10.1007/s12032-020-01372-y

Spiegel, A., Brooks, M. W., Houshyar, S., Reinhardt, F., Ardolino, M., Fessler, E., . . W Weinberg, R. A. (2016). Neutrophils Suppress Intraluminal NK Cell-Mediated Tumor Cell Clearance and Enhance Extravasation of Disseminated Carcinoma Cells. Cancer Discovery, 6(6), 630. doi:10.1158/21598290.CD-15-1157

Strauss, R., Li, Z.-Y., Liu, Y., Beyer, I., Persson, J., Sova, P., . . Lieber, A. (2011). Analysis of epithelial and mesenchymal markers in ovarian cancer reveals phenotypic heterogeneity and plasticity. PLoS One, 6(1), e16186-e16186. doi:10.1371/journal.pone.0016186

Strayer, D. R., Carter, W. A., \& Brodsky, I. (1986). Familial occurrence of breast cancer is associated with reduced natural killer cytotoxicity. Breast Cancer Res Treat, 7(3), 187-192. doi:10.1007/bf01806249

Sulaiman, S. A., Ab Mutalib, N. S., \& Jamal, R. (2016). miR-200c Regulation of Metastases in Ovarian Cancer: Potential Role in Epithelial and Mesenchymal Transition. Front Pharmacol, 7, 271. doi:10.3389/fphar.2016.00271

Takashima, K., Oshiumi, H., Takaki, H., Matsumoto, M., \& Seya, T. (2015). RIOK3-Mediated Phosphorylation of MDA5 Interferes with Its Assembly and Attenuates the Innate Immune Response. Cell Rep, 11(2), 192-200. doi:10.1016/j.celrep.2015.03.027

Terry, S., Buart, S., Tan, T. Z., Gros, G., Noman, M. Z., Lorens, J. B., . . Chouaib, S. (2017). Acquisition of tumor cell phenotypic diversity along the EMT spectrum under hypoxic pressure: Consequences on susceptibility to cell-mediated cytotoxicity. Oncoimmunology, 6(2), e1271858. doi:10.1080/2162402X.2016.1271858

Terry, S., Savagner, P., Ortiz-Cuaran, S., Mahjoubi, L., Saintigny, P., Thiery, J. P., \& Chouaib, S. (2017). New insights into the role of EMT in tumor immune escape. Mol Oncol, 11(7), 824-846. doi:10.1002/1878-0261.12093

Varma, S., Cao, Y., Tagne, J.-B., Lakshminarayanan, M., Li, J., Friedman, T. B., . . Ramirez, M. I. (2012). The Transcription Factors Grainyhead-like 2 and NK2-Homeobox 1 Form a Regulatory Loop That Coordinates Lung Epithelial Cell Morphogenesis and Differentiation. J Biol Chem, 287(44), 37282-37295. doi:10.1074/jbc.M112.408401

Viel, S., Marçais, A., Guimaraes, F. S., Loftus, R., Rabilloud, J., Grau, M., . . W Walzer, T. (2016). TGF- $\beta$ inhibits the activation and functions of NK cells by repressing the mTOR pathway. Science signaling, 9(415), ra19. doi:10.1126/scisignal.aad1884

Vyas, Y. M., Mehta, K. M., Morgan, M., Maniar, H., Butros, L., Jung, S., . . Dupont, B. (2001). Spatial organization of signal transduction molecules in the NK cell immune synapses during MHC class I-regulated noncytolytic and cytolytic interactions. J Immunol, 167(8), 4358-4367. doi:10.4049/jimmunol.167.8.4358

Wang, X. X., Fu, L., Li, X., Wu, X., Zhu, Z., \& Dong, J. T. (2011). Somatic mutations of the mixed-lineage leukemia 3 (MLL3) gene in primary breast cancers. Pathol Oncol Res, 17(2), 429-433. doi:10.1007/s12253-010-9316-0

Wei, S. C., Fattet, L., Tsai, J. H., Guo, Y., Pai, V. H., Majeski, H. E., . . Yang, J. (2015). Matrix stiffness drives epithelial-mesenchymal transition and tumour metastasis through a TWIST1-G3BP2 mechanotransduction pathway. Nature Cell Biology, 17(5), 678-688. doi:10.1038/ncb3157 
Weirich, S., Kudithipudi, S., Kycia, I., \& Jeltsch, A. (2015). Somatic cancer mutations in the MLL3-SET domain alter the catalytic properties of the enzyme. Clin Epigenetics, 7, 36. doi:10.1186/s13148015-0075-3

Werner, S., Frey, S., Riethdorf, S., Schulze, C., Alawi, M., Kling, L., .. A Assmann, V. (2013). Dual roles of the transcription factor grainyhead-like 2 (GRHL2) in breast cancer. The Journal of biological chemistry, 288(32), 22993-23008. doi:10.1074/jbc.M113.456293

Wiesel, M., Crouse, J., Bedenikovic, G., Sutherland, A., Joller, N., \& Oxenius, A. (2012). Type-I IFN drives the differentiation of short-lived effector CD8+ T cells in vivo. European Journal of Immunology, 42(2), 320-329. doi:10.1002/eji.201142091

Wong, J. J., Pung, Y. F., Sze, N. S., \& Chin, K. C. (2006). HERC5 is an IFN-induced HECT-type E3 protein ligase that mediates type I IFN-induced ISGylation of protein targets. Proc Natl Acad Sci U S A, 103(28), 10735-10740. doi:10.1073/pnas.0600397103

Wu, H.-T., Zhong, H.-T., Li, G.-W., Shen, J.-X., Ye, Q.-Q., Zhang, M.-L., \& Liu, J. (2020). Oncogenic functions of the EMT-related transcription factor ZEB1 in breast cancer. Journal of Translational Medicine, 18(1), 51. doi:10.1186/s12967-020-02240-z

Wu, X., Dao Thi, V. L., Huang, Y., Billerbeck, E., Saha, D., Hoffmann, H.-H., . . Rice, C. M. (2018). Intrinsic Immunity Shapes Viral Resistance of Stem Cells. Cell, 172(3), 423-438.e425. doi:10.1016/j.cell.2017.11.018

Xu, D., Li, J., Li, R. Y., Lan, T., Xiao, C., \& Gong, P. (2019). PD-L1 Expression Is Regulated By NF-KB During EMT Signaling In Gastric Carcinoma. Onco Targets Ther, 12, 10099-10105. doi:10.2147/OTT.S224053

Yang, J., Tian, B., Sun, H., Garofalo, R. P., \& Brasier, A. R. (2017). Epigenetic silencing of IRF1 dysregulates type III interferon responses to respiratory virus infection in epithelial to mesenchymal transition. Nat Microbiol, 2, 17086. doi:10.1038/nmicrobiol.2017.86

Yang, Z., Wu, D., Chen, Y., Min, Z., \& Quan, Y. (2019). GRHL2 inhibits colorectal cancer progression and metastasis via oppressing epithelial-mesenchymal transition. Cancer Biol Ther, 20(9), 1195-1205. doi:10.1080/15384047.2019.1599664

Yao, C., Ni, Z., Gong, C., Zhu, X., Wang, L., Xu, Z., ... Zhu, S. (2018). Rocaglamide enhances NK cellmediated killing of non-small cell lung cancer cells by inhibiting autophagy. Autophagy, 14(10), 1831-1844. doi:10.1080/15548627.2018.1489946

Yilmaz, A. S., Ozer, H. G., Gillespie, J. L., Allain, D. C., Bernhardt, M. N., Furlan, K. C., . . Toland, A. E. (2017). Differential mutation frequencies in metastatic cutaneous squamous cell carcinomas versus primary tumors. Cancer, 123(7), 1184-1193. doi:10.1002/cncr.30459

Yu, Y., Huang, R., Zong, X., He, X., \& Mo, W. (2016). INFa-2b inhibitory effects on CD4+CD25+FOXP3+ regulatory $T$ cells in the tumor microenvironment of $\mathrm{C} 57 \mathrm{BL} / 6 \mathrm{~J}$ mice with melanoma xenografts. BMC Cancer, 16(1), 397. doi:10.1186/s12885-016-2473-0 